\title{
Iterative Approach on Redesigning and Construction of a Pipe Inspection Robot
}

by

Aleksandar Ristic

A thesis

submitted to the Victoria University of Wellington in fulfilment of the requirements for the degree of

Master of Science

in Electronics and Computer Systems Engineering.

Victoria University of Wellington

2009 


\begin{abstract}
A pipe inspection robot is a device that is inserted into pipes to check for obstructions or damage. These robots are traditionally manufactured offshore, are extremely expensive, and are often not adequately supported in the event of malfunction. This had resulted in Associated Environmental Services Limited, a New Zealand utiliser of this equipment, facing significant periods of down-time as they wait for their robots to be repaired. Recently, they were informed that several of their robots were no longer supported.

At their instigation, this project was conceived to redesign the electronics and control system of one of these pipe inspection robots, utilising the existing mechanical platform. Requirements for the robot were that it must operate reliably in confined, dark and wet environments, and provide a human user with a digital video feed of the internal status of the pipes. This robot should, as much as possible, incorporate off-the-shelf components, facilitating cheap, and potentially on-site repair.

This project details the redesign and construction of such a robot. It employs three electronic boards integrated with the mechanical components and provides video feedback via a custom graphical user interface. Although at the prototype stage, the electronic redesign has been successful, with a cost of less than a tenth of the original robot purchase price.
\end{abstract}




\section{Acknowledgments}

I would like to thank a number of people for their support and assistance throughout this project.

To Dale Carnegie, my project supervisor, I am thankful for all of his guidance throughout this project.

To Bryan Pobar, my current employer who has helped me financially and supplied the necessary equipment when required.

Many thanks to Johnny McClymont and Jason Edwards with all of the electronics help they have given me throughout the year and it is greatly appreciated and will never be forgotten. Thanks to Scott Forbes, Alan Rennie and Manu Blakiston for the assistance in fine details of this project. To my fellow students; Thomas Roehr and Adrian Jongenelen who helped me with general problems regarding the project and kept me sane during the long hours spent at the university.

I would also like to thank my family for providing me with mental and physical support throughout my studies at Victoria University. 


\section{Contents}

1 Introduction 1

1.1 System Proposal . . . . . . . . . . . . . . . . . . . . . . . 1

1.2 Project Specifications . . . . . . . . . . . . . . . . . . 2

1.3 Project Management $\ldots \ldots \ldots \ldots \ldots \ldots$

1.4 Thesis Structure $\ldots \ldots \ldots \ldots$

2 PIR Background and System Design 5

$2.1 \quad$ Different Types of PIR . . . . . . . . . . . . . . . 5

2.1 .1 The Mole Crawler . . . . . . . . . . . . . . . . . . 6

2.1 .2 The Gopher Crawler . . . . . . . . . . . . . . . . . 6

2.1 .3 The Wolverine Crawler . . . . . . . . . . . . 7

2.1 .4 The Rovver 600 . . . . . . . . . . . . . . . . . . . 8

2.2 The Original System … . . . . . . . . . . . . . . 8

2.2 .1 Base Station . . . . . . . . . . . . . . . . . . . 9

2.2 .2 Micro-controller. . . . . . . . . . . . . . . . . . 11

2.2 .3 Video Imaging . . . . . . . . . . . . . . . . 11

2.2 .4 Power Management \& Motors . . . . . . . . . . . . 13

2.2 .5 Motor Drivers \& Motor Encoders. . . . . . . . . . . . 14

2.3 The New System Design . . . . . . . . . . . . . . . . . . . . 15

2.3 .1 Micro-controller . . . . . . . . . . . . . . . . . . 15

2.3 .2 Motor Drivers . . . . . . . . . . . . . . . . . . . 16

2.3 .3 Power Supply . . . . . . . . . . . . . . . . . . . . . . . 19

2.3 .4 Communication \& Control $\ldots \ldots \ldots$. . . . . . . 20

2.3 .5 Camera Head . . . . . . . . . . . . . . . . . . . . . 22

3 The Chassis 24

3.1 Camera Housing . . . . . . . . . . . . . . . . . . . 24 
3.2 Body \& Connection Cable . . . . . . . . . . . . . . . . . 26

3.2 .1 Connection Cable . . . . . . . . . . . . . . . 28

3.3 Motors . . . . . . . . . . . . . . . . . . . . . . . . . . . 29

3.4 Gears \& Chain Drive . . . . . . . . . . . . . . . . . . 31

3.4 .1 Gears . . . . . . . . . . . . . . . . 31

3.4 .2 Chain Drive . . . . . . . . . . . . . . . 34

3.5 Motor Encoders . . . . . . . . . . . . . . . 34

$\begin{array}{lll}4 & \text { Hardware } & 38\end{array}$

4.1 Power Source . . . . . . . . . . . . . . . . . . . . . . . . 38

4.2 Motor Controller Board . . . . . . . . . . . . . . . . . . . . . 41

4.2.1 Sabertooth 5 A Dual Motor Driver . . . . . . . . . . 41

4.2 .2 Micro-controller AT89C51AC3 . . . . . . . . . . . 45

4.2 .3 Main Board RS-485 Transceiver . . . . . . . . . . . . 47

4.2 .4 SP3238 Transceiver . . . . . . . . . . . . . . 47

4.3 Camera EVI-370 \& Camera Board . . . . . . . . . . . . . . . 48

4.3 .1 Camera EVI-370 . . . . . . . . . . . . . . . . . . . . 49

4.3 .2 Camera Board . . . . . . . . . . . . . . . . . . . 49

4.3 .3 Light Emitting Diodes . . . . . . . . . . . . . . . 51

4.4 Base Station Board . . . . . . . . . . . . . . . . . 52

$4.4 .1 \quad$ FT232BQ USB UART . . . . . . . . . . . . . . . 52

4.4 .2 Base Station RS-485 Transceiver. . . . . . . . . . . . 53

4.4 .3 Video Capture Card . . . . . . . . . . . . . . 55

5 Software 57

5.1 Serial Communication . . . . . . . . . . . . . 58

5.1 .1 LabVIEW Serial Transmit . . . . . . . . . . . . . . . . 59

5.1 .2 Micro-controller Serial Initialisation . . . . . . . . . 63

5.1.3 Micro-controller Automatic Address Recognition . . 65

5.1 .4 Micro-controller Serial Receive . . . . . . . . . . . 66

5.1 .5 Micro-controller Serial Transmit . . . . . . . . . . . . 68

5.1 .6 LabVIEW Serial Receive . . . . . . . . . . . . . . . . . 71

5.2 LabVIEW Image Acquisition . . . . . . . . . . . . . . . 72

5.3 Micro-controller Motor Control . . . . . . . . . . . . . 73

$5.3 .1 \quad$ PCA Initialisation . . . . . . . . . . . . . 73 
$5.3 .2 \quad$ PWM Output \& Control . . . . . . . . . . . . 76

\begin{tabular}{lll}
\hline 6 & Results & 79
\end{tabular}

6.1 Printed Circuit Boards . . . . . . . . . . . . . . . . 79

6.1 .1 Version One . . . . . . . . . . . . . . . 79

6.1 .2 Version Two . . . . . . . . . . . . . . 81

6.1 .3 Version Three . . . . . . . . . . . . . . 83

6.1 .4 Version Four . . . . . . . . . . . . . . . . 86

6.2 Motor Characteristics . . . . . . . . . . . . . . . . . . . . . 88

6.3 System Characteristics . . . . . . . . . . . . . . . . . . . 90

6.3 .1 No Load . . . . . . . . . . . . . . . . . 993

6.3 .2 Full Load . . . . . . . . . . . . . . . . . . . 94

6.4 System Performance $\ldots \ldots$. . . . . . . . . . . . . 95

$\begin{array}{llr}7 & \text { Conclusion } & 98\end{array}$

7.1 Project Review . . . . . . . . . . . . . . . . . . . . . . . . 98

7.2 Future Work . . . . . . . . . . . . . . . . . . . . . . . 99

$7.2 .1 \quad$ Power Supply . . . . . . . . . . . . . . . . . . . . 99

7.2 .2 Motor Encoders . . . . . . . . . . . . . . . . . . . . 100

7.2 .3 Internal Camera Enclosure . . . . . . . . . . . . . 101

7.2 .4 Cable Reel . . . . . . . . . . . . . . . . . . . . . . . . . 101

7.3 Summary . . . . . . . . . . . . . . . . . . 102

$\begin{array}{ll}\text { Glossary } & 108\end{array}$

\begin{tabular}{ll}
\hline List of Appendices & 110
\end{tabular}

\begin{tabular}{ll}
\hline A Hardware & 110
\end{tabular}

A.1 Parts . . . . . . . . . . . . . . . . . . . . 110

A.2 Parts List One . . . . . . . . . . . . . . . . . . . . . . . . . . 111

A.3 Parts List Two . . . . . . . . . . . . . . . . . . . . . . . . . 112

A.4 Parts List Three . . . . . . . . . . . . . . . . . . . . . . . . 113

A.5 Main Board Micro-controller. . . . . . . . . . . . . . . . . . . 114

A.6 Main Board Power Supply . . . . . . . . . . . . . . . . . . . . 115

A.7 Main Board SP3238 . . . . . . . . . . . . . . . . . . . . . . 115

A.8 Main Board RS-485 . . . . . . . . . . . . . . . . . . . . . 116 
A.9 Main Board Circuit Protection . . . . . . . . . . . . . . . . 116

A.10 Camera Board . . . . . . . . . . . . . . . . . . 117

A.11 Base Station Board . . . . . . . . . . . . . . . 118

\begin{tabular}{ll}
\hline B Software & 119
\end{tabular}

B.1 Micro-controller Configuartion . . . . . . . . . . . . . . . 120

B.2 Micro-controller ASCII To Decimal . . . . . . . . . . . . . . . 121

B.3 Micro-controller ASCII To Hexadecimal 1 . . . . . . . . . . . 122

B.4 Micro-controller ASCII To Hexadecimal 2 . . . . . . . . . . . 123

B.5 Micro-controller Serial Interrupt . . . . . . . . . . . . . . . 124

B.6 Micro-controller PCA Configuration . . . . . . . . . . . . . . 125

B.7 Micro-controller Serial Functions . . . . . . . . . . . . 126

B.8 Micro-controller Main Function 1 . . . . . . . . . . . . . . 127

B.9 Micro-controller Main Function 2 . . . . . . . . . . . . . . . 128

B.10 Micro-controller Main Function 3 . . . . . . . . . . . . . . 129 


\section{List of Tables}

2.1 Switching Devices . . . . . . . . . . . . . . . . . . 14

2.2 Bridge Drivers $\ldots \ldots \ldots \ldots \ldots$

2.3 Micro-Controllers . . . . . . . . . . . . . . . . . . . . . 16

2.4 H-Bridge Drivers . . . . . . . . . . . . . . . . . . 17

2.5 Motor Driver Boards . . . . . . . . . . . . . . . . . . 18

2.6 DC to DC converters . . . . . . . . . . . . . . . . . . . . . 19

2.7 Differential Transceivers . . . . . . . . . . . . . . . . . 21

3.1 Motors Characteristics . . . . . . . . . . . . . . . 30

3.2 Motor Gear Head Characteristics . . . . . . . . . . . . 30

5.1 Camera Commands and Packets $\ldots . . \ldots \ldots 1$ 


\section{List of Figures}

2.1 The Mole Crawler . . . . . . . . . . . . . . . . . . . . . . . 6

2.2 The Gopher Crawler . . . . . . . . . . . . . . . . . . 7

2.3 The Wolverine Crawler . . . . . . . . . . . . . . . . . . 7

2.4 Rovver $600 \ldots \ldots \ldots \ldots$. . . . . . . . . . . . . . . 8

2.5 Power Supply \& Transformer . . . . . . . . . . . . . . . . . . 9

2.6 Joystick Peripheral . . . . . . . . . . . . . . . . . . . . . 10

2.7 Old Micro-controller Board . . . . . . . . . . . . . . . . . 12

2.8 Camera EVI-370 . . . . . . . . . . . . . . . . . . . . . . 12

2.9 Old Camera Board . . . . . . . . . . . . . . . . . . . 13

2.10 AT89C51AC3 Micro-controller . . . . . . . . . . . . 16

2.11 Mini DC to DC Converter . . . . . . . . . . . . . . . 20

2.12 FCB-EX1010 Camera . . . . . . . . . . . . . . . . 22

3.1 Left: Camera Housing Right: Camera Top. . . . . . . . . . . 25

3.2 Left: Rotating Pivot Right: Complete Camera. . . . . . . . . 25

3.3 Rotating Camera Shaft . . . . . . . . . . . . . . . . . . 26

3.4 Robot Brass Body . . . . . . . . . . . . . . . . . . 27

3.5 Back End of the Robot $\ldots \ldots \ldots \ldots$

3.6 Cable Pin Out . . . . . . . . . . . . . . . . . . . . . . . . 28

3.7 Motor Encoder Configuration . . . . . . . . . . . . . . . 32

3.8 Camera Head Y Axis Configuration . . . . . . . . . . . . 33

3.9 Chain Drive Configuration. . . . . . . . . . . . . . . . . 34

3.10 2RM360 Encoder . . . . . . . . . . . . . . . . . . 35

3.11 2RM360 Output Signals . . . . . . . . . . . . . . 35

3.12 Second Motor Encoder Configuration . . . . . . . . . . 36

3.13 Camera Head Y Axis Gear Configuration . . . . . . . . . 36 
4.1 V300B12C250BL DC to DC Converter . . . . . . . . . 38

4.2 Left: DC Coupled Interface Right: AC Coupled Interface . . 39

$4.3 \quad$ Left: Trim Down Right: Trim Up . . . . . . . . . . . . . . . . . 40

4.4 DC to DC Converter Pin Configuration . . . . . . . . . . 40

4.5 Sabertooth 5 A Dual Motor Driver . . . . . . . . . . . . . . 41

4.6 Sabertooth Motor Configuration . . . . . . . . . . . . . . . 43

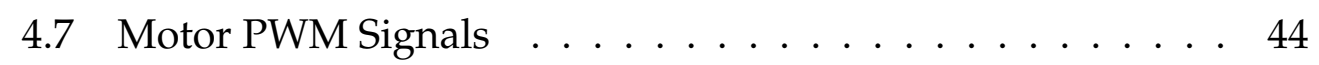

4.8 Motor Circuit Protection . . . . . . . . . . . . . . . . . . . 44

4.9 Motor Drivers 1 \& $2 \ldots \ldots \ldots$

$4.10 \mathrm{RX}$ and TX Double Switch . . . . . . . . . . . . . 46

4.11 LTC1387 Pin Configuration . . . . . . . . . . . . . . . 48

4.12 Camera Board Setup . . . . . . . . . . . . . . . . 50

4.13 Camera Circuit Protection . . . . . . . . . . . . . . . 50

4.14 FT232BQ Configuration . . . . . . . . . . . . . . . 53

4.15 RS-485 Configuration . . . . . . . . . . . . . . . . . . 54

4.16 FT232BQ and RS-485 Configuration . . . . . . . . . . . 54

4.17 Purple: TXEND Blue: RS-485 RX Yellow: FT232BQ RXD . . 55

4.18 AVerMedia USB Video Capture Card . . . . . . . . . . . 56

5.1 LabVIEW GUI Design . . . . . . . . . . . . . . . . . . . . . . . . 57

5.2 LabVIEW GUI Block Diagram . . . . . . . . . . . . . . . . . . 58

5.3 VISA Block Diagram Initialisation . . . . . . . . . . . . . . 59

5.4 Data Packet Structure . . . . . . . . . . . . . . . . . . . 60

5.5 Motor Speed Values. . . . . . . . . . . . . . . . . . . . 60

5.6 Camera \& Micro-controller Button Panel. . . . . . . . . . . 62

5.7 Transmit Data Block Diagram . . . . . . . . . . . . . . 62

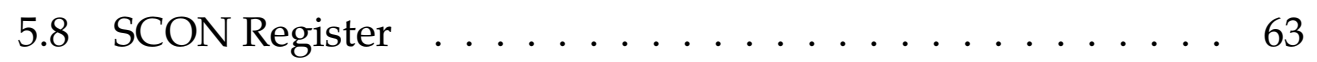

5.9 TMOD Register $\ldots \ldots \ldots$. . . . . . . . . . . . . . . . 63

5.10 PCON Register . . . . . . . . . . . . . . . . . . . . 65

5.11 Serial Connection Initialisation . . . . . . . . . . . . 65

5.12 Automatic Address Recognition . . . . . . . . . . . 66

5.13 Serial Communication Initilisation . . . . . . . . . . . 67

5.14 Serial Interrupt Routine Part 1 . . . . . . . . . . . . . . 67

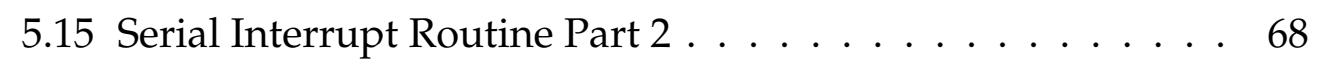

5.16 RS-485 Configuration . . . . . . . . . . . . . . . . . . . 69 
LIST OF FIGURES Ix

5.17 RS-485 Transmitting Data . . . . . . . . . . . . . . 70

5.18 Data Transmit Routine . . . . . . . . . . . . . . . . . . 70

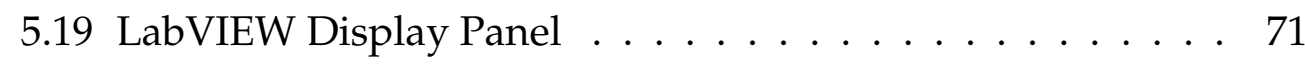

5.20 Receive Data Block Diagram . . . . . . . . . . . . . . . 72

5.21 Block Diagram Video Acquistion . . . . . . . . . . . . . . 72

5.22 CCAPM Register . . . . . . . . . . . . . . 73

5.23 IEN0 Register . . . . . . . . . . . . . . . . . . 74

5.24 CMOD Register . . . . . . . . . . . . . . . . . 74

5.25 PCA Initialisation . . . . . . . . . . . . . . . . . . 75

5.26 PCA Timer 0 Configuration . . . . . . . . . . . . 75

5.27 Motors, Camera, Serial and PCA Initialisation . . . . . . . 76

5.28 Motor Speed Calculation \& Output . . . . . . . . . . . . 77

5.29 Motor Drivers Error Message . . . . . . . . . . . . . . . 78

5.30 Camera Power \& Communication Error Message. . . . . . . 78

6.1 Motor Driver Board Version One . . . . . . . . . . . . . . . . 80

6.2 Motor Driver Board Schematic Version One . . . . . . . . . . 81

6.3 Motor Driver Board Version Two . . . . . . . . . . . . . . . 82

6.4 Motor Driver Board Schematic Version Two . . . . . . . . . . 83

6.5 Main Driver Board Version Three . . . . . . . . . . . . . . . . 84

6.6 Camera Board Version Three . . . . . . . . . . . . . . . . . . 84

6.7 Base Station Board Version Three . . . . . . . . . . . . . . . 85

6.8 Main Motor Board Version Four . . . . . . . . . . . . . . . . 86

6.9 Base Station Board Version Four . . . . . . . . . . . . . . . 87

6.10 Camera Board Version Four . . . . . . . . . . . . . . . . . 88

6.11 Current Drawn - No Load . . . . . . . . . . . . . . . . . . . . 89

6.12 Current Drawn - Full Load . . . . . . . . . . . . . . . . . . . . 90

6.13 Voltage vs. RPM - No Load . . . . . . . . . . . . . . . . . . 91

6.14 Transmitted Value v.s. PWM Pulse Width . . . . . . . . . . . 91

6.15 Terminal Voltage Difference . . . . . . . . . . . . . . . . 92

6.16 Current Drawn - No Load . . . . . . . . . . . . . . . . . 93

6.17 Power - No Load . . . . . . . . . . . . . . . . . . . . 94

6.18 Current Drawn - Full Load . . . . . . . . . . . . . . . . . . . . 95

6.19 Power - Full Load . . . . . . . . . . . . . . . . . . . . . . . . . 96

6.20 Forward and Backward Speed - Full Load . . . . . . . . . . . 97 
7.1 Left: ARM Right: FARM . . . . . . . . . . . . . . . . . 100

7.2 Motorised Reel . . . . . . . . . . . . . . . . . . . . . 103 


\section{Chapter 1}

\section{Introduction}

\subsection{System Proposal}

Water, gas and sewerage pipes are used everyday in nearly all modern households. These pipes are usually constructed out of copper, concrete, ceramics and occasionally plastic. The biggest problem that is encountered inside these pipes is deterioration, which can lead to leaky or burst pipes and in turn could put public safety in jeopardy.

The New Zealand environmental services have different approaches and techniques on how to examine such pipes. The techniques include ultrasonic, radiography, eddy current (electromagnetic induction) and remote visual inspection. The ultrasonic, radiography and eddy current techniques require the pipe surface to be accessible. The pipes in the real world are usually located underground which makes them harder to maintain. The remote visual inspection technique is preferable because the pipe can remain underground and if any damage is detected the pipe can be replaced without the need for exposing the whole length of the pipe. This technique uses a large variety of gadgets which range from small bore video scopes to large pipe inspection robots. The small bore video scopes are used for short or vertical distances and can be lowered down the pipe. For long horizontal distances it is preferred to use pipe inspection robots because of their internal drive mechanism.

The pipe inspection robots are used in harsh environments and due to wear and tear the internal electronics become faulty and stop working 
over time. Most suppliers (several are listed in Chapter 2) who manufacture these robots are located overseas and if a problem is encountered the robot needs to be shipped, resulting in a large amount of down time. To remove this unnecessary down-time a New Zealand based company called Associated Environmental Services (AES) is developing a pipe inspection robot, and could manufacture and maintain such robots.

There are two ways of achieving such an outcome: either to construct the robots by "re-inventing the wheel", meaning every part inside the robot would have to be custom designed, or by using off-the-shelf parts which would be advantageous when it comes to debugging and replacing components. This project will use off-the-shelf parts which can be easily replaced if broken and the operational information is easily accessible via the internet.

\subsection{Project Specifications}

The primary objective of the project is to design the electronics for a pipe inspection robot including an operator interface for remote control. The off-the-shelf components purchased and implemented have to meet the hardware and software design specifications as follows:

Usability: The software interface implemented will need to be easy to use with little or no operator training. The software has to provide the user with full control over the robot and feedback to allow for necessary corrections if problems are found.

Extensibility: At software and hardware levels the design must be expandable to allow for future developments which might require new functions/circuits to be implemented or removed.

Generic Application: The overall system needs to be reusable and allow the user to execute the same task on multiple platforms. Future projects would adopt the same software and hardware designs to produce multiple working robots.

Robustness: The hardware design needs to perform well under stress and 
unforeseen circumstances. The software must deal with invalid user input and inform the user if a fault has occurred.

Reliability: The hardware and software designs have to be reliable to perform in the working environment for up to three hours at a time.

\subsection{Project Management}

To meet the specifications mentioned in Section 1.2 the project needs to be managed in simple steps with regards to time and money. This project sets the following milestones to track progress and success of the development stages.

1. Research current and available pipe inspection robots.

2. Become familiar with the mechanical aspects of the robot, power supply and chassis.

3. Research and select the hardware devices and software interface packages.

4. Design and construct the hardware devices

5. Design and implement the software interface

6. Test and evaluate

The final result needs to be an operational pipe inspection robot which can traverse inside a pipe and allow an operator to visually detect internal damage. The user will need remote control over the robot with real-time data and instant feedback.

\subsection{Thesis Structure}

The thesis is presented as follows:

Chapter 2 discusses some different pipe inspection robots that are available and the different capabilities of each robot. The project's hardware, software and power design considerations are also discussed. 
Chapter 3 analyses the chassis used to hold all of the electrical and mechanical components, and the different assembly steps required to assemble the robot.

Chapter 4 discusses the hardware implementation to construct the fully operational pipe inspection robot.

Chapter 5 analyses the low level software design as well as the techniques used to enable the user to remotely control the robot.

Chapter 6 discusses the electronic boards that have been constructed and the steps taken to make the robot robust. This chapter also presents the results of the power tested boards, along with an analysis of any limitations found.

Chapter 7 concludes the project achievements, discusses the possible and required future work and provides a project summary. 


\section{Chapter 2}

\section{PIR Background and System Design}

The pipe inspection robot was provided by Associated Environmental Services Limited in a non-functioning condition. The mechanical gears, motors and chains were all in working order but the electronic components did not operate. Possible solutions were either to reverse engineer the electronics or perform a complete redesign. Given the age of the existing robot, it was decided a complete redesign of the robot's electronics was necessary, using modern components and technology.

\subsection{Different Types of PIR}

There are different types of Pipe Inspection Robots (PIR) that are produced by companies all over the world. These companies include RoboProbe Technologies [1], Electromechanica Inc. [2], GE Inspection Technologies [3], Radiodetection [4], Triventek A/S [5] and Angelus Research [6]. Essential differences between the robots are the different size wheels to give them better clearance and the quality of camera employed, high resolution cameras can spot even very small problems in a dark environment. 


\subsubsection{The Mole Crawler}

The Mole Crawler robot [7] shown in Figure 2.1 [8] is manufactured by RoboProbe Technologies. It is very similar in size and shape to the one that we are working on. This robot is designed for pipes with a diameter between 10 and $30 \mathrm{~cm}$. The dimensions of the robot are $20 \times 7.6 \times 7.6 \mathrm{~cm}$. The robot's body can be constructed out of anodised aluminium or brass and is powered by a $48 \mathrm{~V} \mathrm{DC}$ motor. The robot is a four wheel drive and comes with different sized wheels providing different clearance levels.

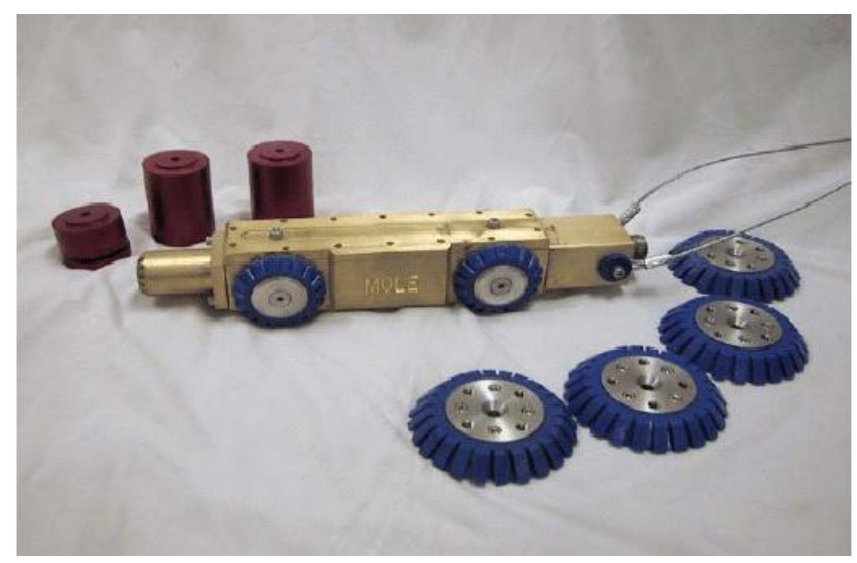

Figure 2.1: The Mole Crawler

\subsubsection{The Gopher Crawler}

The intermediate sized Gopher Crawler [9] shown in Figure 2.2 [10] is manufactured by RoboProbe Technologies and it has three different sized wheels for different clearance levels. The robot is designed to carry a standard or a pan-tilt camera and to fit inside a pipe with a diameter between 15 and $60 \mathrm{~cm}$. The difference between the two cameras is the pan-tilt camera has the capability to rotate along two axes while the normal camera can only rotate along one axis. The Gopher is best suited for distances around $150 \mathrm{~m}$, any longer and a more powerful robot would have to be deployed in order to drag the weighty cable behind it. 


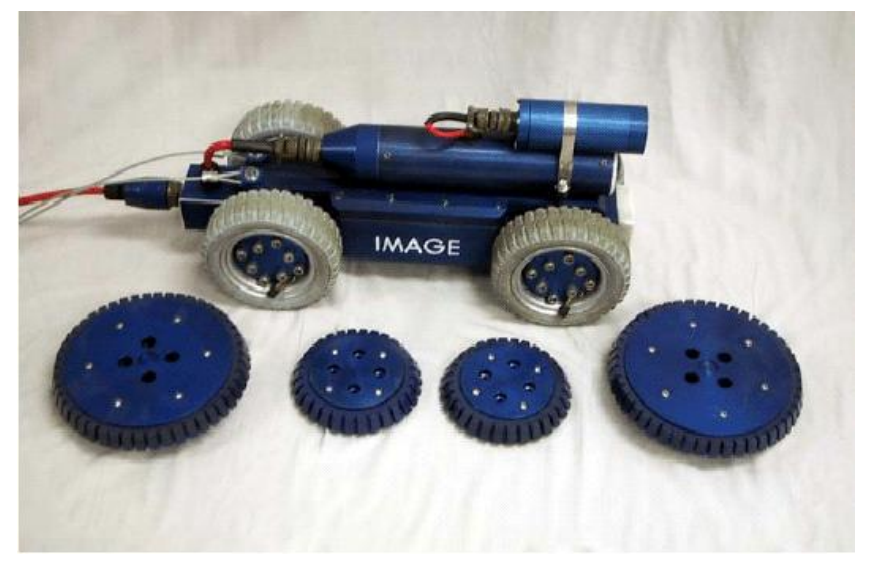

Figure 2.2: The Gopher Crawler

\subsubsection{The Wolverine Crawler}

The Wolverine Crawler [11] is the largest robot out of the three robots manufactured by RoboProbe Technologies shown in Figure 2.3[12]. This robot can operate in pipes between 20 and $84 \mathrm{~cm}$ in diameter with use of different sized wheels. The camera carried can either be a standard or pantilt, depending on the circumstances. The Wolverine possesses dual motor drive which enables steerable operation.

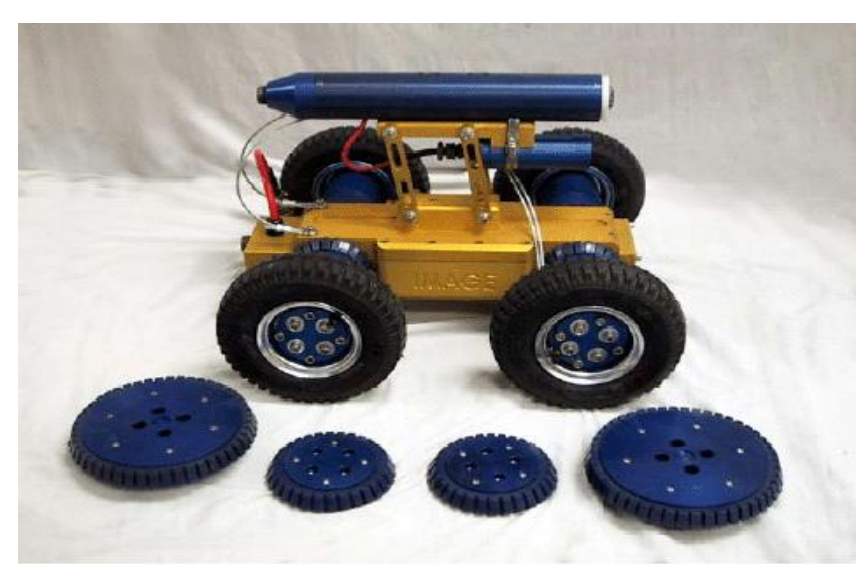

Figure 2.3: The Wolverine Crawler 


\subsubsection{The Rovver 600}

The Rovver 600 robotic crawler shown in Figure 2.4 [13] is manufactured also by GE Inspection Technologies and measures $282 \times 118 \times 90 \mathrm{~mm}$. The robot weighs $8 \mathrm{~kg}$ and is constructed out of nickel plated brass, stainless steel and aluminium. The crawler contains $2 \times 20 \mathrm{~W}$ DC motors to provide the user with a steerable 4 -wheel drive robot. The robot can be purchased with three different sets of wheels which provide the robot with different clearance levels for different sized pipes. The $86 \mathrm{~mm}$ diameter wheels are designed for $11.5 \mathrm{~cm}$ pipes and the 110 and $135 \mathrm{~mm}$ diameter wheels are used for 20 and $22.5 \mathrm{~cm}$ pipes respectively.

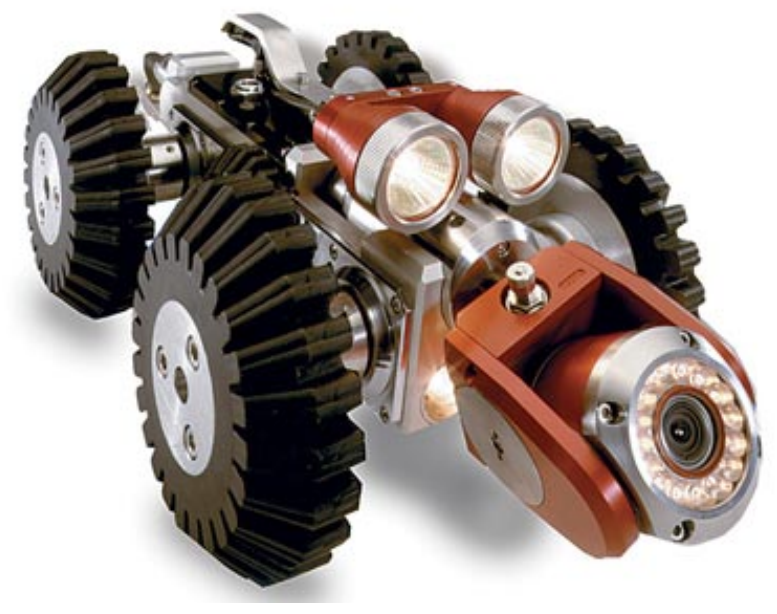

Figure 2.4: Rovver 600

A colour camera with a resolution of 380,000 pixels is used to display the transmitted video image to the user at the base station. In total $90 \mathrm{~W}$ is consumed to power the $2 \times 20 \mathrm{~W}$ auxiliary lights, $2 \times 20 \mathrm{~W}$ tractor lights and $16 \times 0.6 \mathrm{~W}$ camera lights.

\subsection{The Original System}

The original robot is contained within a brass chassis and is powered by a power box located at the base station. A power box delivers $240 \mathrm{~V}$ DC to the robot over a $400 \mathrm{~m}$ long cable. The robot contains three $12 \mathrm{~V} \mathrm{DC}$ 
motors manufactured by the Faulhaber Group [14]. The front of the robot contains a Sony EVI-370 camera, four light bulbs and a camera board. Inside the body of the robot a DC to DC converter is used to power all of the electronics, motors and motor encoders. The external measurements of the robot measure $520 \times 90 \times 80 \mathrm{~mm}$ and the internal compartment which hosts the electronics and motors has dimensions $390 \times 70 \times 70 \mathrm{~mm}$. Parts purchased and boards made have to be functional, but small in size.

\subsubsection{Base Station}

The base station consists of an isolating transformer, power supply, TV screen, hand operated joystick and a keyboard. The power box MCA150 and all of the peripherals are manufactured by OPTIMESS Engineering. The power box is connected to an isolating transformer which plugs directly to the mains. The remaining peripherals receive appropriate power levels by connecting directly to the power box. The power box is used to convert $240 \mathrm{~V}$ AC to $240 \mathrm{~V} \mathrm{DC}$ which is then sent to the robot using a long cable. The overall base station configuration can be seen in Figure 2.5 .

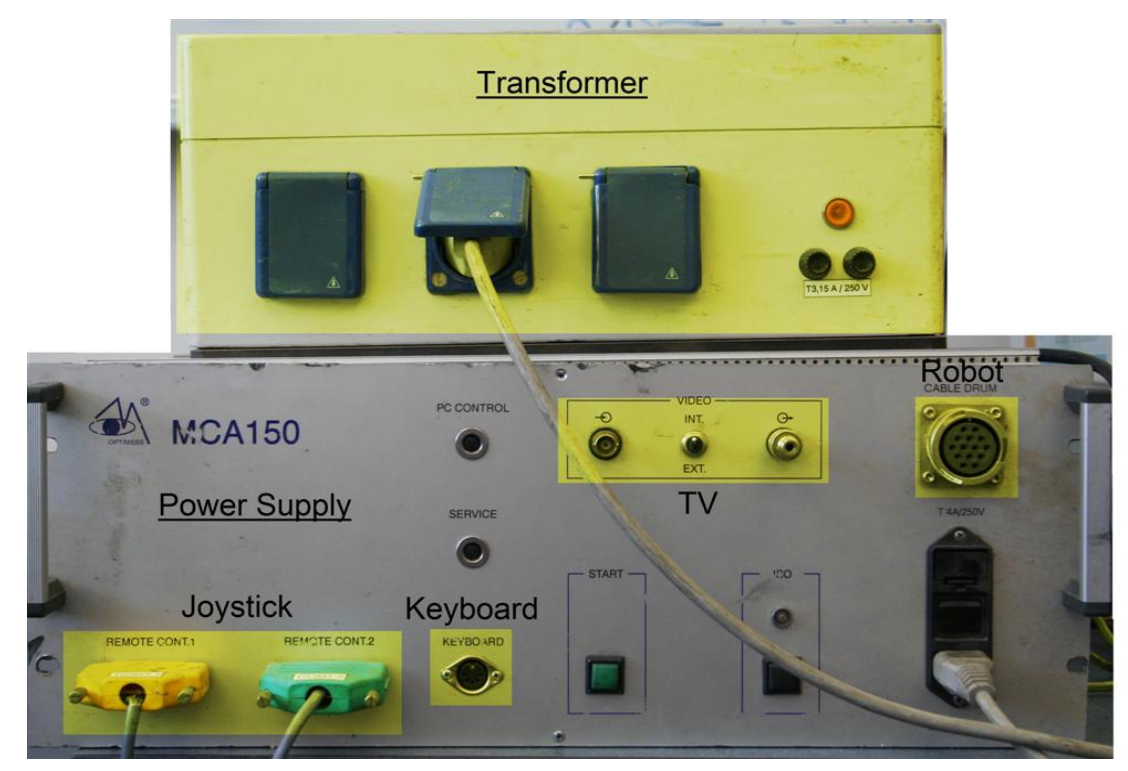

Figure 2.5: Power Supply \& Transformer

To provide the power box with $240 \mathrm{~V}$ AC a switch has to be turned on which is located near the back of the transformer. The yellow light bulb 
located at the front of the transformer informs the user if everything is in working condition. If the light does not turn on this means a problem has been encountered and the transformer should be looked at before continuing with the configuration.

When the power box has been connected to the transformer all of the peripherals should be connected before applying the power. When the power has been turned on, the light bulb located at the front of the power box will turn red. This means that the power box is receiving $240 \mathrm{~V} \mathrm{AC}$ but no power is being delivered to the robot. To deliver $240 \mathrm{~V}$ DC to the robot the user has to set the "ON/OFF" switch in the on position which is located on the joystick peripheral as shown in Figure 2.6.

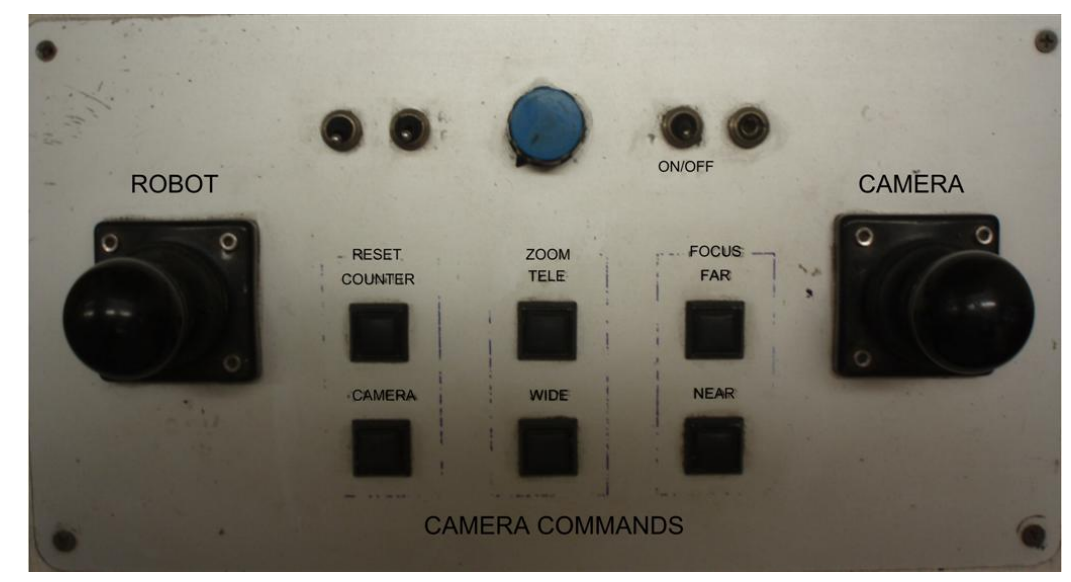

Figure 2.6: Joystick Peripheral

When the switch has been set by the user, the power box has to be turned off for 30 seconds before turning it back on. When the power has been restored, the light bulb located at the front should turn green which confirms that the power box is in working condition and there is a $240 \mathrm{~V}$ DC output to the robot. If the light bulb remains red this means a power box problem has been encountered and previous actions should be repeated or the manufacturer should be contacted to resolve the problem.

The joystick peripheral is able to control the camera and the robot simultaneously. The user can therefore control the camera lens with the zoom in and out buttons and use the focus buttons to adjust the camera lens. 


\subsubsection{Micro-controller}

The micro-controller found inside the robot on a small board is a 16-bit Toshiba TMP96PM40F. This contains $32 \mathrm{~KB}$ of ROM and $1 \mathrm{~KB}$ of RAM memory with a nominal operating voltage of $5 \mathrm{~V}$. The micro-controller also incorporates the following features:

- 10 bit Analogue to Digital Converter (ADC)

- 8-bit/16-bit Timer/Counter

- UART/SIO

- PWM Output

- Pattern Generator

The micro-controller uses dual channel UART to communicate with the camera on one channel and uses the other channel to communicate with the base station computer. However, the micro only possesses a dual channel PWM output port. This is not desirable because the robot contains three motors, therefore only two motors can be controlled. The microcontroller board also contains one PALCE16V8 programmable logic array (PAL) and two AM29F010 1 MB external memory chips as shown in Figure 2.7

\subsubsection{Video Imaging}

The camera located inside the robot head is a Sony EVI-370 as shown in Figure 2.8. The EVI-370 is a digital block camera and is powered by a $8 \mathrm{~V}$ power supply. The difference between the digital block camera and a normal digital camera is the physical size. A normal camera is contained inside a plastic case which provides the user control buttons and functions. A digital block camera is contained inside a small metal square which does not have buttons for the user to interact with but rather provides the capability to be controlled from a computer. The camera uses Video System Control Architecture (VISCA) to communicate with the computer. The camera also incorporates the following features: 


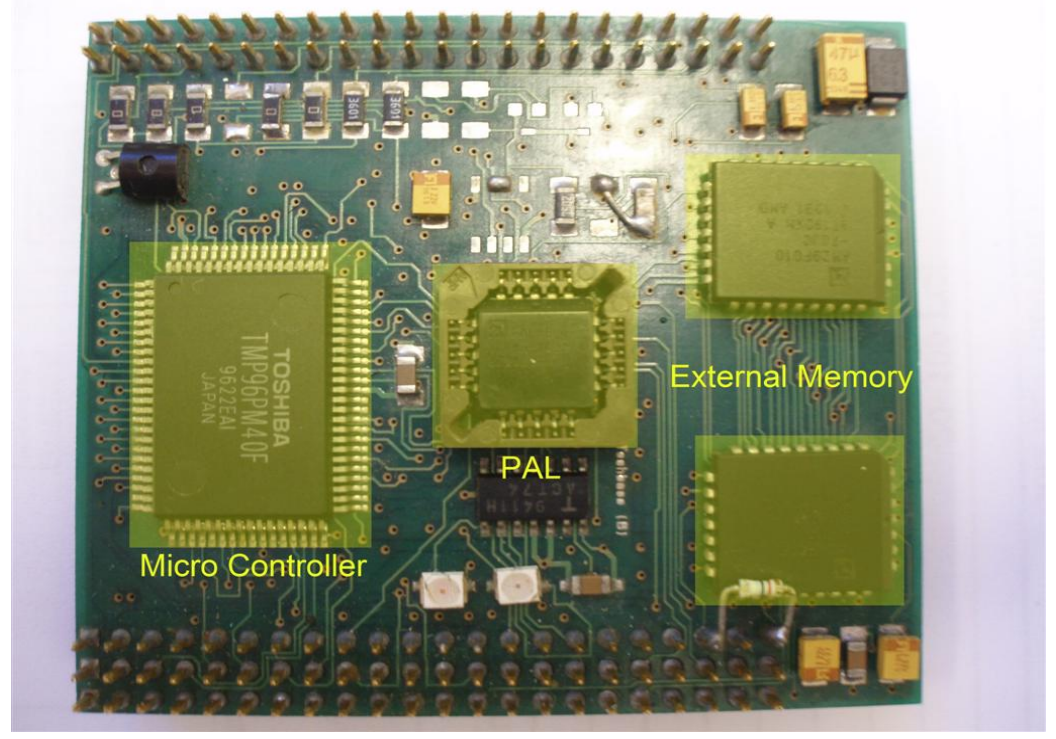

Figure 2.7: Old Micro-controller Board

- $12 \times$ Optical Zoom, High Speed Auto Focus Lens

- On-Screen Display

- Analogue Zoom Position Feedback

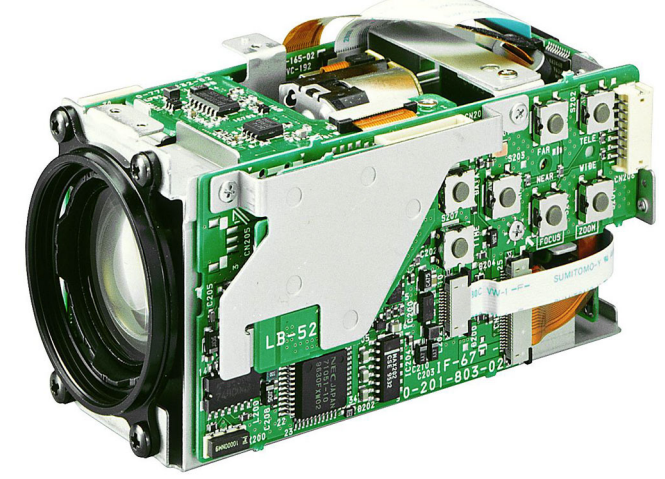

Figure 2.8: Camera EVI-370

The power delivered to the camera was via a camera board located in a compartment above the camera and away from the main circuitry. The camera board is powered by the DC to DC converter (Section 2.2.4) which 
is located within the robot. The converter delivers $12 \mathrm{~V}$ DC to the camera board and then it is stepped down to $8 \mathrm{~V} \mathrm{DC}$ with the use of a voltage regulator. The camera board is relatively small due to the room constraint and measures $65 \times 34 \mathrm{~mm}$. The board is attached to the camera head using two screws but the voltage regulator is attached to the outer walls of the camera head to dissipate more heat as shown in Figure 2.9.

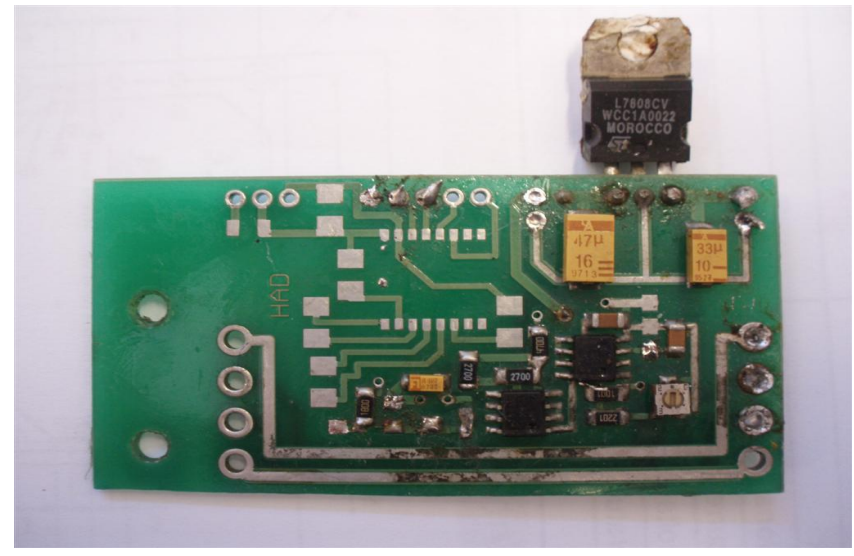

Figure 2.9: Old Camera Board

\subsubsection{Power Management \& Motors}

The on-board DC to DC converter is manufactured by ETA Electric Industry Co. Ltd [15]. The converter has an input voltage range between 220 and $400 \mathrm{~V}$ DC and an output voltage of $12 \mathrm{~V}$ DC. The converter connects to the power box via the connection cable which receives $240 \mathrm{~V}$ DC and produces $240 \mathrm{~W}$ of power. The maximum output current is $20 \mathrm{~A}$, which is above the required current for the robot. The converter is attached to a metal plate which is then attached to the robot's chassis to dissipate heat. The converter is small in size and measures $90 \times 60 \times 13 \mathrm{~mm}$.

The two 2225U012S motors are used to control the camera head. One motor rotates the head along the $\mathrm{X}$ axis and the other motor rotates the head along the $\mathrm{Y}$ axis. They require a $12 \mathrm{~V}$ input voltage and have a terminal resistance of $8.71 \Omega$ as specified in the datasheet. The third motor is a $3557 \mathrm{~K} 012 \mathrm{CR}$ and is used to drive the robot in the forward and reverse 
directions. The motor also uses a $12 \mathrm{~V}$ power supply, has a terminal resistance of $2.4 \Omega$ and has output power of $14.5 \mathrm{~W}$. This motor is larger in size and carries a larger load compared to the other two motors.

\subsubsection{Motor Drivers \& Motor Encoders}

The motor driver circuits used to control the main drive motors and camera motors are constructed using switching devices and bridge drivers. The main motor driver is constructed using the L6202 bridge driver and BUZ345 MOSFET. The camera motors are controlled using the HIP4081 bridge driver and IRLIZ44N MOSFET. The comparison between the bridge drivers and switching devices are detailed in Table 2.1 and 2.2 .

Table 2.1: Switching Devices

\begin{tabular}{|l|c|c|c|c|}
\hline & $\operatorname{Max} V_{D S}(V)$ & $\operatorname{Max} I_{D}(\mathrm{~A})$ & $R_{D S}(\Omega)$ & Gate Threshold $(\mathrm{V})$ \\
\hline BUZ345 & 100 & 41 & 0.045 & 3 \\
\hline IRLIZ44N & 55 & 30 & 0.022 & 1.5 \\
\hline
\end{tabular}

As seen in Table 2.1 the BUZ345 MOSFET has a higher drain to source voltage and current. This is because the BUZ345 is used to drive the main drive motor which has a higher payload compared to the IRLIZ44N. Similar comparisons are seen with bridge drivers as shown in Table 2.2. The L6202 bridge driver used to control the main motor has a higher supply voltage and output current, compared to the HIP4081 driver which has a lower payload.

Inside the robot there are three motor encoders which are discussed in detail in Section 3.5. The motor encoder used to count the camera head revolutions along the $\mathrm{Y}$ axis is constructed using a spur gearing configuration. The other two motor encoders are used to count the number of revolutions the camera rotates along the $X$ axis. The first motor encoder is 2 RM360 which is manufactured by SCANCON [16]. The 2RM360 uses single shot pulses to inform the micro-controller about the current position of the camera head. The second motor encoder is a single toggle switch 
Table 2.2: Bridge Drivers

\begin{tabular}{|l|c|c|c|c|}
\hline & Max $V_{C C}(\mathrm{~V})$ & $\begin{array}{c}\text { Logic I/O } \\
\text { Voltages (V) }\end{array}$ & $\begin{array}{c}\text { Output } \\
\text { Current }(\mathrm{mA})\end{array}$ & Input Channels \\
\hline HIP4081 & 16 & $V_{C C}+0.3$ & 0.9 & 1 \\
\hline L6202 & 48 & $2-7$ & 1.5 & 2 \\
\hline
\end{tabular}

which sends a high pulse to the micro-controller each time the rotating $X$ axis shaft has completed a single rotation.

\subsection{The New System Design}

The old implemented system had some good circuit designs which could be re-used inside the new one, but the opportunity was taken to determine if new devices might be more suitable.

\subsubsection{Micro-controller}

The micro-controller for the redesigned robot has to possess the following features: at least one RS-232 communication port, three PWM outputs and two external interrupts. Several micro-controllers were considered and analysed for functionality and these were ATMega128, AT91AM7X256 and AT89C51AC3. The ATMega128 and AT89C51AC3 were considered because of the author's previous experience in dealing with these devices and having the software library implemented and available on the computer. The AT91AM7X256 micro-controllers were left over from a previous project and therefore some experimental testing was done. All three micro-controllers are manufactured by Atmel Corporation [17] and their similarities and differences can be seen in Table 2.3 .

Initially the ATMega128 micro-controller was considered but problems were encountered with the software library and because of this it was disregarded. In the end the AT89C51AC3 micro-controller was chosen as 
Table 2.3: Micro-Controllers

\begin{tabular}{|l|c|c|c|}
\hline & ATMega128 & AT89C51AC3 & AT91AM7X256 \\
\hline $\begin{array}{l}\text { Number of PWM } \\
\text { channels }\end{array}$ & 8 & 5 & 4 \\
\hline $\begin{array}{l}\text { Number of USART } \\
\text { channels }\end{array}$ & 2 & 1 & 2 \\
\hline External Interrupts & Yes & Yes & Yes \\
\hline Flash Memory (Kb) & 128 & 64 & 256 \\
\hline Operating Voltages (V) & $2.7-5.5$ & $3-5.5$ & $3-3.6$ \\
\hline SPI Communication & Yes & Yes & Yes \\
\hline
\end{tabular}

shown in Figure 2.10. The device possessed all of the required features and the author had access to a fully working development board.

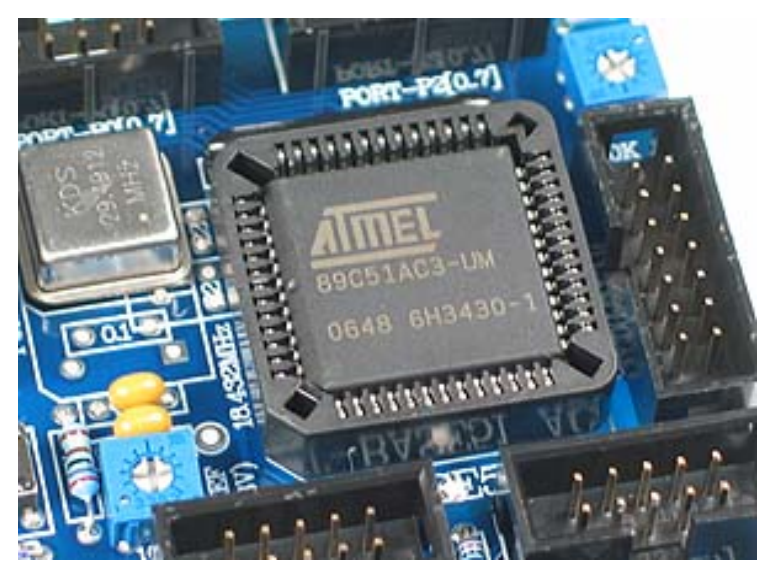

Figure 2.10: AT89C51AC3 Micro-controller

\subsubsection{Motor Drivers}

In this section the new motor drivers that are available to be purchased off-the-shelf are compared to the old components that are used and implemented in the previous design as the HIP4081. Our essential choices are either to purchase an H-bridge driver and a switching device to construct 
a custom motor driver board, or to purchase a commercially available motor driver which is fully built and tested.

Several $\mathrm{H}$-bridges were investigated. The main specifications that were considered were the supply voltage, current drawn and the number of signal inputs. Three H-bridge drivers that were considered for the project and their specifications are shown in Table 2.4 .

Table 2.4: H-Bridge Drivers

\begin{tabular}{|l|c|c|c|}
\hline & HIP4081 & TLE6284 & L6206D \\
\hline Supply Voltage (V) & $5-16$ & $7.5-60$ & $8-52$ \\
\hline Supply Current (A) & 2.5 & 0.85 & 2.8 \\
\hline Number of Signal Inputs & $1-4$ & 2 & $1-4$ \\
\hline Internal Switching Devices & No & No & Yes \\
\hline
\end{tabular}

The reason for choosing the HIP4081 chip is because other students have used it and recommended it as a reliable driver. The TLE6284 chip was a free sample sent from a distributor and it was considered after testing the device to see if it would integrate with the current system. The L6206D device was considered because it contains internal switching devices and therefore the physical space constraint would be less of an issue.

Initially the L6206 driver was chosen and implemented due to having internal switching devices, but was disregarded because a large amount of heat was being dissipated and this would decrease the life expectancy of the chip. The reason for disregarding the TLE6284 chip was because the supply current is rated at $0.85 \mathrm{~A}$ and the total current drawn by all three motors is around 1.6 A. In the end the HIP4081 bridge driver was chosen which could deliver $2.5 \mathrm{~A}$ of current and be controlled with one input signal.

The HIP4081 driver does not have internal switching devices and for this reason switching devices had to be selected, purchased and implemented. When selecting the switching devices such as IGBTs the collector to emitter voltage and current must be checked to make sure the motors will not exceed these values. If MOSFETs are considered the drain current 
and drain to source voltage should be monitored.

The switching devices that were considered for implementation were FGPF7N60 and PC40F IGBTs because of the similar voltage levels compared to the ones used in the previous design. The PC40F is designed to drive small motors such as the ones located inside the camera head. The device has a collector current of $7 \mathrm{~A}$ and a maximum collector to emitter voltage of $600 \mathrm{~V}$. The second device FGPF7N60 is more powerful and would be used to power the main motor which would need a higher current supply. This device is capable of delivering a constant current of $27 \mathrm{~A}$ at a voltage level of $600 \mathrm{~V}$. These devices become considerably hot and would have to be attached to the brass frame of the robot to dissipate the heat.

The supplier that was considered to purchase the off-the-shelf motor driver boards was Dimension Engineering [18], who produce boards of small dimensions that can handle a large current. The manufacturer has different boards available and the specifications are shown in Table 2.5 .

Table 2.5: Motor Driver Boards

\begin{tabular}{|l|c|c|c|c|}
\hline & SyRen10 & SynRen25 & $\begin{array}{c}\text { Sabertooth } \\
\text { 2X5 }\end{array}$ & $\begin{array}{c}\text { Sabertooth } \\
2 \text { X10 }\end{array}$ \\
\hline $\begin{array}{l}\text { Supply } \\
\text { Voltage (V) }\end{array}$ & $6-18$ & $6-24$ & $6-18$ & $6-18$ \\
\hline $\begin{array}{l}\text { Supply } \\
\text { Current (A) }\end{array}$ & 10 & 25 & 5 & 10 \\
\hline $\begin{array}{l}\text { Peak } \\
\text { Current (A) }\end{array}$ & 15 & 45 & 10 & 15 \\
\hline Weight (g) & 26 & 55 & 19 & 60 \\
\hline Size (mm) & $35 \times 57 \times 14$ & $61 \times 58 \times 21$ & $45 \times 40 \times 13$ & $59 \times 75 \times 17$ \\
\hline
\end{tabular}

All of the motor driver boards listed can be powered by $12 \mathrm{~V}$ DC. The amount of current that can be handled by the motor drivers exceeds the expected amount of current that will be drawn by our motors which is around 1.6 A and therefore the Sabertooth2X5 was considered. The Sabertooth $2 \mathrm{X} 5$ has the ability to drive two motors simultaneously while deliv- 
ering $5 \mathrm{~A}$ to each motor. The motor drivers can be used in different modes such as the R/C micro-controller which controls the motors using a PWM signal and packetised serial which controls the motor drivers via a serial connection.

\subsubsection{Power Supply}

One option for developing a new power supply was to introduce on-board rechargeable batteries which would provide the necessary power to the robot. The motors used inside the robot have a $12 \mathrm{~V}$ DC input and therefore the rechargeable batteries would have to have a $12 \mathrm{~V}$ DC output, be small enough to be placed inside the robot and have a high current capability. The rechargeable batteries were eliminated because the robot is connected to the power box via a umbilical cable which delivers $240 \mathrm{~V} \mathrm{DC}$ and therefore the old electrical power design is considered by using a DC to $\mathrm{DC}$ converter.

The DC to DC converter has to be small in size, have an output voltage of $12 \mathrm{~V} \mathrm{DC}$, input voltage of $240 \mathrm{~V}$ DC and provide enough current to power all of the electronics and motors. The supplier chosen for the DC to DC converter was Vicor Power [19]. In total five different types of converters were considered and these are the maxi, mini, micro, VI-200 and VI-J00 series. The comparison between different types and models can be seen in Table 2.6.

Table 2.6: DC to DC converters

\begin{tabular}{|l|c|c|c|c|}
\hline Model & $\begin{array}{c}\text { Input } \\
\text { Voltage (V) }\end{array}$ & $\begin{array}{c}\text { Output } \\
\text { Voltage (V) }\end{array}$ & $\begin{array}{c}\text { Output } \\
\text { Power (W) }\end{array}$ & Dimensions (mm) \\
\hline Maxi & $24-375$ & $2-54$ & $160-600$ & $117 \times 55.9 \times 12.7$ \\
\hline Mini & $24-375$ & $2-54$ & $100-300$ & $57.9 \times 55.9 \times 12.7$ \\
\hline Micro & $24-375$ & $2-54$ & $50-150$ & $57.9 \times 36.8 \times 12.7$ \\
\hline VI-200 & $10-400$ & $1-95$ & $50-200$ & $116.9 \times 61 \times 12.7$ \\
\hline VI-J00 & $10-400$ & $1-95$ & $25-100$ & $57.9 \times 61 \times 12.7$ \\
\hline
\end{tabular}

As seen in Table 2.6 all of the models have the ability to step down 
from $240 \mathrm{~V}$ DC down to $12 \mathrm{~V}$ DC. The difference between the models is the output power and size. The reason for not choosing the maxi and VI200 series is because the physical dimensions are too large to fit inside the robot and therefore the mini series was chosen because the output power is larger compared to the micro and VI-J00 series.

The mini series has similar electrical specifications as the one used in the previous design and therefore it was chosen and purchased as shown in Figure 2.11.

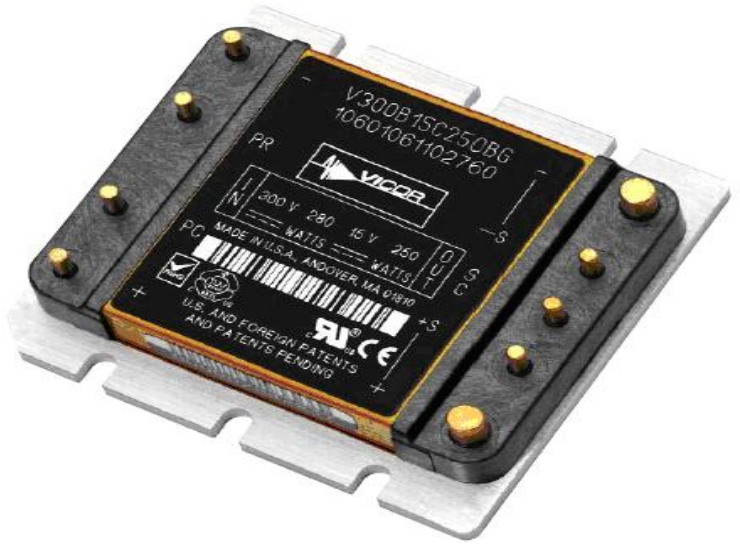

Figure 2.11: Mini DC to DC Converter

\subsubsection{Communication \& Control}

The communication between the base station and the robot must be reliable and the standards that were considered were:

- Wireless

- Universal Serial Bus (USB)

- FireWire

- Single-Ended Drivers (RS-232 \& RS423)

- Differential Drivers (RS-485 \& RS422) 
The wireless communication was eliminated due to signal waves travelling down a pipe would have signal reflections and thus resulting in noise being introduced into the circuit. The distance between the base station and the robot can reach up to $400 \mathrm{~m}$ and therefore the RS-232 was eliminated because the maximum distance that can be reached is $15 \mathrm{~m}$. For this reason FireWire and USB were also eliminated because the maximum distance is 4.5 and $5 \mathrm{~m}$ respectively. Therefore a differential driver had to be chosen and Table 2.7[20] lists different types that were considered.

Table 2.7: Differential Transceivers

\begin{tabular}{|c|c|c|c|}
\hline Specifications & RS-423 & RS-422 & RS-485 \\
\hline Maximum cable length & $1200 \mathrm{~m}$ & $1200 \mathrm{~m}$ & $1200 \mathrm{~m}$ \\
\hline $\begin{array}{l}\text { Receiver input voltage } \\
\text { range }\end{array}$ & $+/-12 \mathrm{~V}$ & $\begin{array}{l}-10 \mathrm{~V} \text { to } \\
+10 \mathrm{~V}\end{array}$ & $\begin{array}{l}-7 \mathrm{~V} \text { to } \\
+12 \mathrm{~V}\end{array}$ \\
\hline Receiver input sensitivity & $+/-200 \mathrm{mV}$ & $+/-200 \mathrm{mV}$ & $+/-200 \mathrm{mV}$ \\
\hline Maximum data rate & $100 \mathrm{~Kb} / \mathrm{s}$ & $\begin{array}{c}100 \mathrm{~Kb} / \mathrm{s}- \\
10 \mathrm{Mb} / \mathrm{s}\end{array}$ & $\begin{array}{c}100 \mathrm{~Kb} / \mathrm{s}- \\
10 \mathrm{Mb} / \mathrm{s}\end{array}$ \\
\hline $\begin{array}{l}\text { Maximum driver } \\
\text { output voltage }\end{array}$ & $+/-6 \mathrm{~V}$ & $\begin{array}{c}-0.25 \mathrm{~V} \text { to } \\
+6 \mathrm{~V}\end{array}$ & $\begin{array}{l}-7 \mathrm{~V} \text { to } \\
+12 \mathrm{~V} \\
\end{array}$ \\
\hline Driver load impedance $(\Omega)$ & $\geq 450$ & 100 & 120 \\
\hline
\end{tabular}

The difference between a differential transceiver and a single-ended transceiver such as the RS-232 is that the single-ended transceivers send data using a single wire with a common ground wire. The RS-485, RS423 and RS-422 use two wires to transmit the data signal differentially and therefore the larger voltage difference between the signals results in greater noise immunity and a larger reliable cable length. The differential signals are shared by the receive and transmit lines.

In the end the RS-485 transceiver was chosen as the communication device between the robot and the base station because the maximum cable length is over $400 \mathrm{~m}$ and the maximum data rate can reach $10 \mathrm{Mb} / \mathrm{s}$. The device chosen to communicate between the base station board and the computer is the RS-232 chip because the distance is less than $15 \mathrm{~m}$ and the computer has a built in serial connector which outputs RS-232 signal 
levels. The device configuration and signal processing is discussed in Section 4.2.3.

\subsubsection{Camera Head}

The camera located inside the head of the robot is in a working condition but there was no datasheet on how to control it, so therefore purchasing a new camera was considered. The cameras that were considered ranged from web cameras to high resolution digital cameras.

The web cameras are easy to implement and are relatively cheaper in price compared to the digital cameras. The biggest advantage that a web camera has over a digital camera is the size of the camera and the electronic components that come with it. The whole camera can be purchased on a board $40 \times 40 \mathrm{~mm}$. This is a good feature due to a tight room constraint that is inside the head of the robot and therefore providing extra room for other electronics. The problem that is encountered with a web camera is that there is no digital or optical zoom available and therefore the user would not be able to detect very small cracks inside the pipe.

For these reasons a block camera with digital or optical zoom had to be acquired. The camera that was chosen was a FCB-EX1010 from Total Turnkey Solutions [21] as shown in Figure 2.12. The camera has $12 \times$ digital zoom and $36 \times$ optical zoom. The dimensions of the camera are $50 \times$ $57.5 \times 87 \mathrm{~mm}$. The camera is much larger compared to the web camera but small enough to fit inside the robot's head.

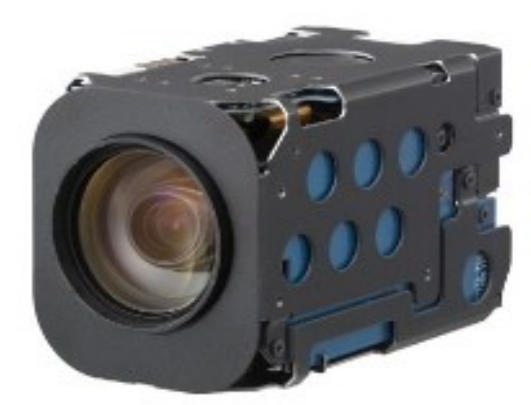

Figure 2.12: FCB-EX1010 Camera 
The FCB-EX1010 was not purchased because the minimum order quantity is 10 and we only required 1 for this project. Therefore it was decided to go with the old EVI-370 camera that was located inside the robot and try to reverse engineer the power and communication lines. 


\section{Chapter 3}

\section{The Chassis}

\subsection{Camera Housing}

The camera housing compartment measures $50 \times 96 \times 45 \mathrm{~mm}$. The camera (EVI-370) is enclosed inside a metal frame and when inserted inside the compartment there is less than $1 \mathrm{~mm}$ of free space around each edge. This creates a very tight fit around the camera minimising damage that might occur from vibrations or shakes. The external measurements of the camera head are $90 \times 129 \times 55 \mathrm{~mm}$. The front of the camera contains 4 LEDs and a glass lens. The top of the camera is sealed with a metal plate using seven screws. The electrical wires for power and communication are passed through a pivot hole located at the top of the camera housing as shown in Figure 3.1 .

If any of the LEDs are damaged, they can easily be replaced by unscrewing the front cover and pulling them out. Special care has to be taken when new LEDs are placed, making sure the polarity of the LED is correct, otherwise they could suffer permanent damage.

The camera pivot is a circular piece of metal. The hole is $15 \mathrm{~mm}$ in diameter and the bottom part of the pivot measures $38 \mathrm{~mm}$ in diameter. The pivot is attached to the top of the camera head using four screws. To minimise wear and tear, the pivot has to be well oiled at all times.

The camera housing is attached to the bottom part of the head using four screws along with a metal plate which also attaches the rotating gears located underneath. The description of the gears and their operation is 

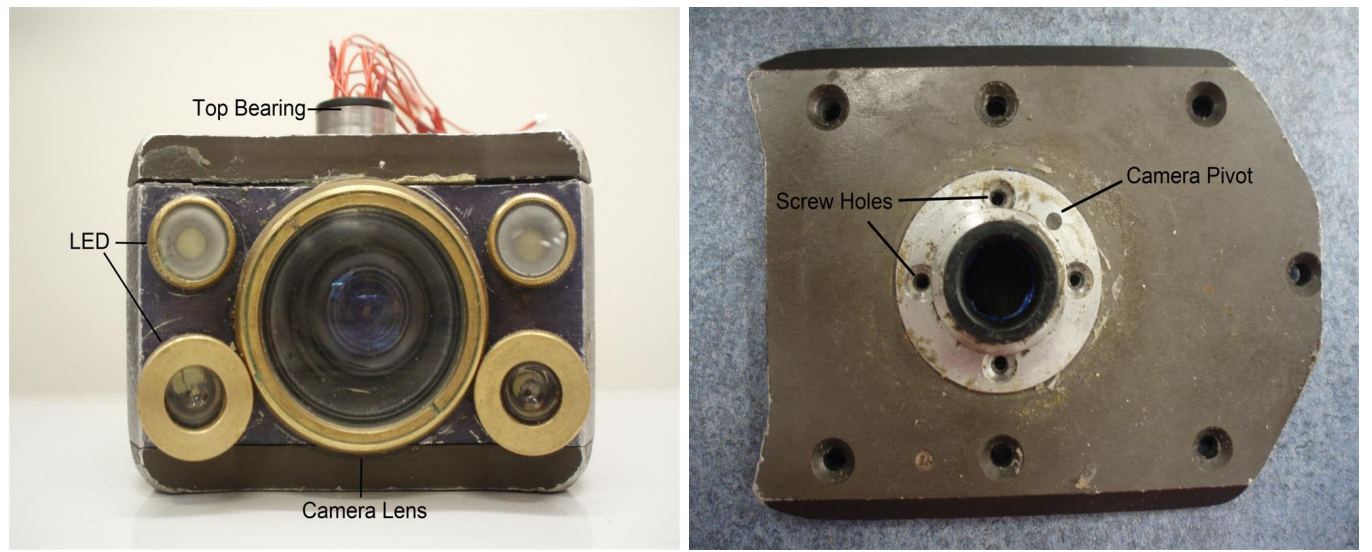

Figure 3.1: Left: Camera Housing Right: Camera Top

discussed in Section 3.4. Both top and bottom compartments are sealed off using twelve screws and thus constructing the whole camera head as seen in Figure 3.2 .
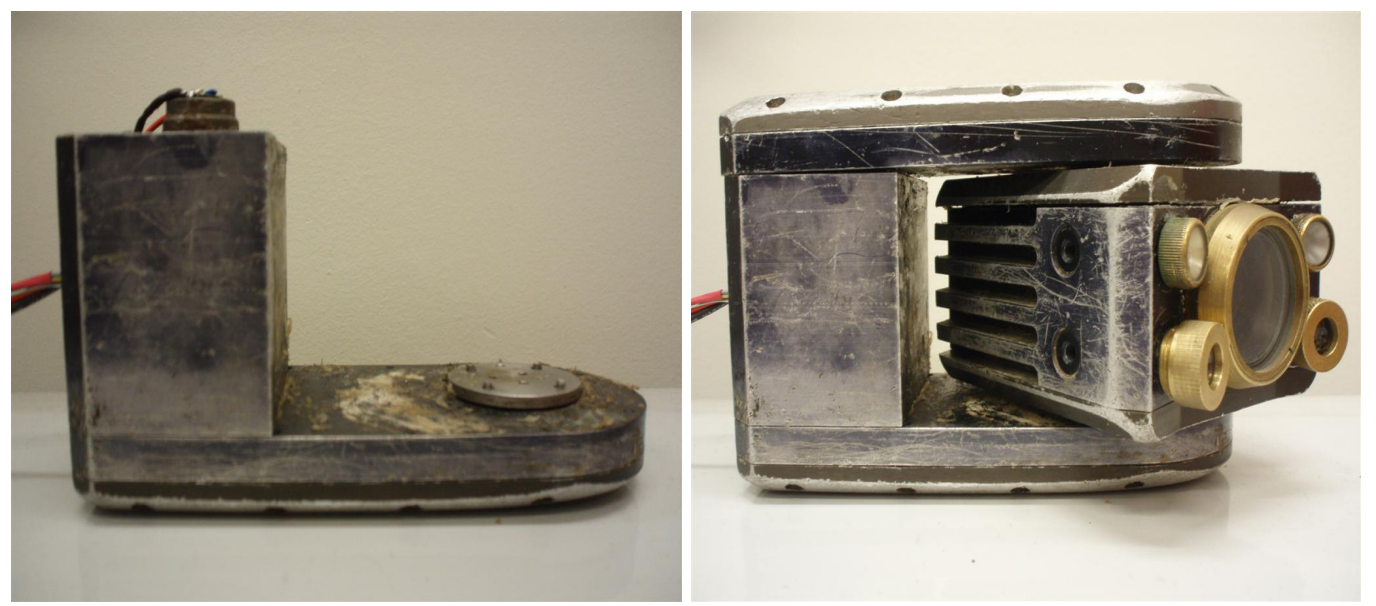

Figure 3.2: Left: Rotating Pivot Right: Complete Camera

The camera head is then attached to the body of the robot using a long rotating shaft. The rotating shaft is attached to the body of the robot with four screws on either side. The rotating shaft measures $132 \mathrm{~mm}$ in length. The exposed camera end measures $48 \mathrm{~mm}$ in diameter and the part that is attached to the rotating gears measures $17 \mathrm{~mm}$ in diameter. The rotating shaft can be seen in Figure 3.3 . 


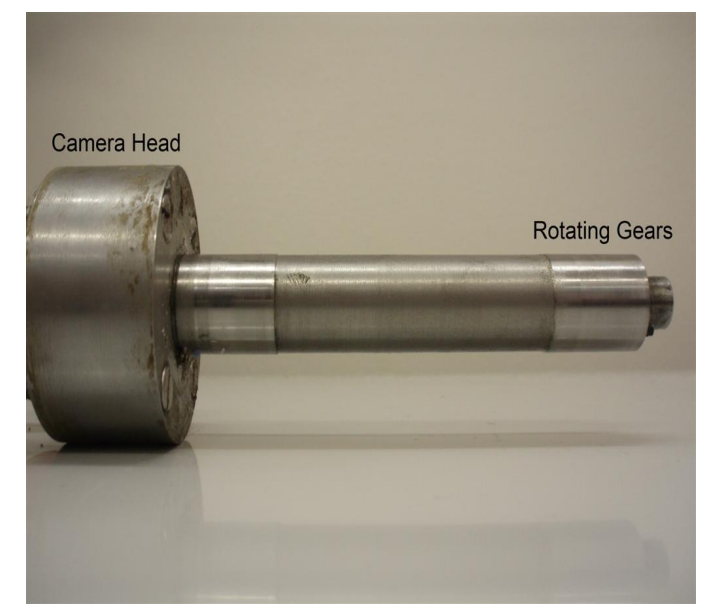

Figure 3.3: Rotating Camera Shaft

\subsection{Body \& Connection Cable}

The brass chassis structure is $10 \mathrm{~mm}$ thick and it measures $320 \mathrm{~mm}$ in length. The front and back of the robot measures $55 \mathrm{~mm}$ in width while the middle of the robot measures $70 \mathrm{~mm}$ as seen in Figure 3.4. The depth measures $70 \mathrm{~mm}$ and the total internal volume of the body is $1379 \mathrm{~cm}^{3}$. The internal compartment contains two motors, two motor encoders, two motor driver circuits, a DC/DC converter, a main electronics board and a drive chain. The original set-up of all of the components inside the body are shown in Appendix A.1-A.4.

Located at the rear of the body is a 10 pin connector which is used to connect the robot with the base station via a cable. The header directly connects to the DC/DC converter, electronics boards, communication lines and the camera. The connection between the components and the main board is passed through a series of small compartments. The compartments are used to strengthen the connection between the connecting cable and the robot. These compartments connect directly to the chassis using four screws as displayed in Figure 3.5. The compartments are $40 \mathrm{~mm}$ in length, $23 \mathrm{~mm}$ wide and $45 \mathrm{~mm}$ deep. The free space available inside the compartment is very small and therefore no electronic equipment can be placed inside.

The wheels are attached to two independent drive shafts. The torque 


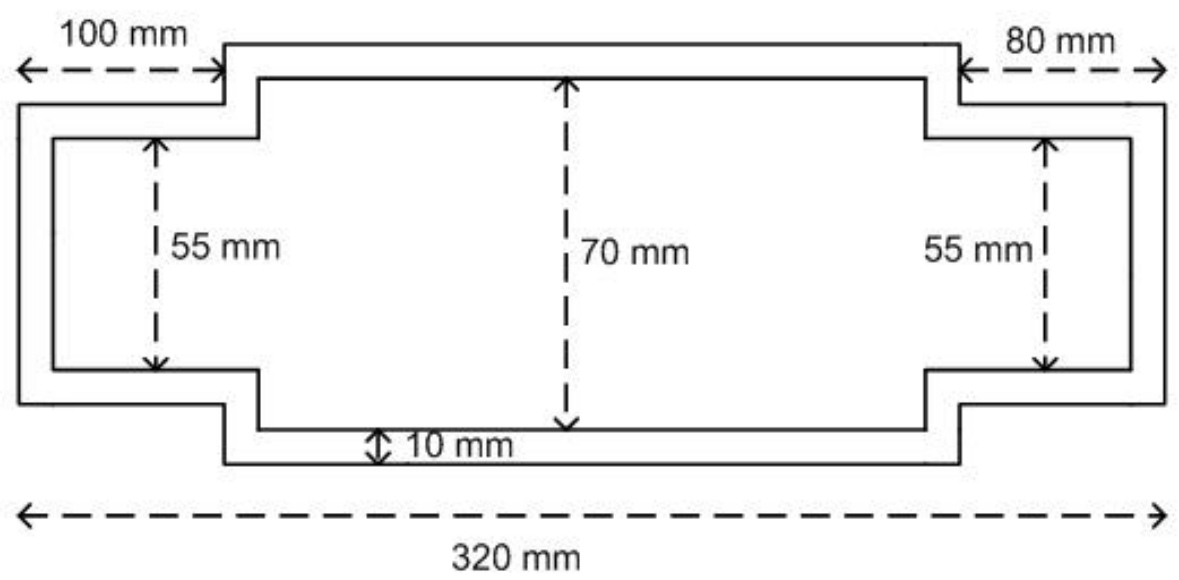

Figure 3.4: Robot Brass Body

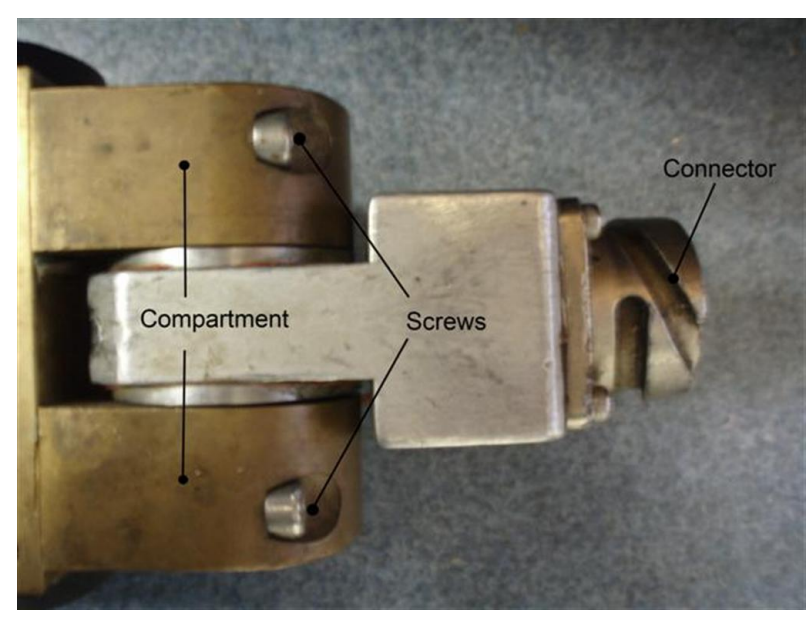

Figure 3.5: Back End of the Robot 
initially produced by the main motor is transferred to the back and front wheels using a pair of gears and a drive chain discussed later in Section 3.4. The wheels measure $60 \mathrm{~mm}$ in diameter and are held in place by two screws. These screws make it possible for the wheels to be replaced if at any time the user wishes to achieve higher clearance or if damage occurs.

\subsubsection{Connection Cable}

The connection cable between the base station and the robot measures $180 \mathrm{~m}$ in length. The cable is constructed using 10 wires that can be used for power, communication, video imagining or anything else the operator might need in the future. The configuration on the pins that attach to the robot connector can be seen in Figure 3.6

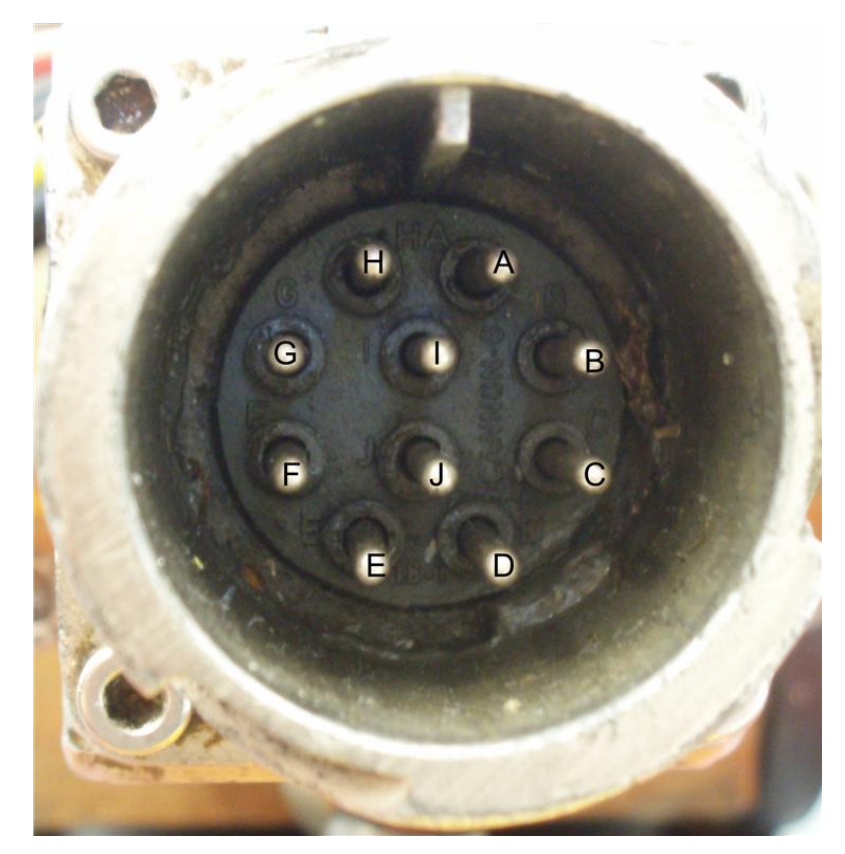

Figure 3.6: Cable Pin Out

In total four lines $\mathrm{A}, \mathrm{B}, \mathrm{C}$ and $\mathrm{H}$ are used to deliver $240 \mathrm{~V}$ DC. This is done to increase the total current that can be delivered when the robot travels longer distances because the robot will get heavier when the cable length increases. The camera requires two wires ( $G \& F)$ which are used 
for the video image and the ground signals. The last four wires (I, J, D $\&$ E) are configured as twisted pair communication lines which improve the efficiency of the transmitted differential signals. This is a restriction on the number of communication lines available. The communication devices have to be carefully chosen and designed not to exceed the maximum number of lines available.

\subsection{Motors}

In total the robot contains three $12 \mathrm{~V} \mathrm{DC}$ motors with gear heads attached. There are two small motors which are used to rotate the camera along the $\mathrm{X}$ and $\mathrm{Y}$ axes and one larger motor which is used to move the robot in forward and reverse directions. All of the motors and gear heads which are used inside the robot are no longer manufactured by the Faulhaber Group and because of this some datasheets for the components are no longer available. These differences are described in the next section.

The two smaller motors used for the head rotations are 2225012SR series. The specifications sheets for these motors are no longer available and therefore the closest available match is the 2224012SR series. The difference between the two motors is the overall length. The 2225012SR measures $25.2 \mathrm{~mm}$ in length while the 2224012SR measures $24.2 \mathrm{~mm}$. The larger motor used to move the robot in forwards and backwards directions is $3557 \mathrm{KO} 12 \mathrm{CR}$. The specifications for this motor are still available and the differences between the two motors can be seen in Table 3.1.

Looking at Table 3.1. the motor used to drive the robot forwards and backwards has a power rating three times higher than the smaller motors. This is necessary due to the higher load being moved. These positive aspects come at a cost with the efficiency, no-load speed and back-EMF constants being larger than the smaller motors. The characteristics listed in Table 3.1 are all a compromise for one advantage over another and no single motor can be perfect for the job. These motors were chosen and implemented because they were used in the previous system and they were in working order.

The gear heads attached to the 2225012SR motors are the 23/1 series 
Table 3.1: Motors Characteristics

\begin{tabular}{|l|c|c|}
\hline & 2224012SR & 3557K012CR \\
\hline Nominal Voltage $(\mathrm{V})$ & 12 & 12 \\
\hline Terminal Resistance $(\Omega)$ & 8.71 & 2.4 \\
\hline Output Power $(\mathrm{W})$ & 4.05 & 14.5 \\
\hline Efficiency $(\%)$ & 82 & 76 \\
\hline No-Load Speed (rpm) & 7800 & 4800 \\
\hline Stall Torque $(\mathrm{mNm})$ & 19.8 & 115 \\
\hline Back-EMF Constant $(\mathrm{mV} / \mathrm{rpm})$ & 1520 & 2450 \\
\hline Weight $(\mathrm{g})$ & 46 & 275 \\
\hline
\end{tabular}

and the gear head attached to the $3557 \mathrm{KO} 12 \mathrm{CR}$ motor is the $30 / 1$ series. Looking at Table 3.2 it is shown that the $30 / 1$ series is designed to handle more torque and have a higher efficiency because it is used to move the robot forwards and backwards. The $23 / 1$ series is used to rotate lighter loads, in this case the camera head, and therefore the operational torque does not need to be as high.

Table 3.2: Motor Gear Head Characteristics

\begin{tabular}{|l|c|c|}
\hline & $\mathbf{2 3 / 1}$ & $\mathbf{3 0 / 1}$ \\
\hline Maximum Input Speed (rpm) & 4000 & 4000 \\
\hline Reduction ratio (nominal) & $592: 1$ & $66: 1$ \\
\hline Gear Weight (g) & 110 & 171 \\
\hline Intermittent Operation Torque (mNm) & 1000 & 2400 \\
\hline Continuous Operation Torque (mNm) & 700 & 1800 \\
\hline Efficiency (\%) & 55 & 70 \\
\hline
\end{tabular}

When the motors and gear heads were being tested a problem was encountered with the $3557 \mathrm{KO} 12 \mathrm{CR}$ motor which could not leave the stall mode unless the user intervened and turned it. Upon closer examination it was noticed that the motor had to be replaced due to a faulty coil. 
To purchase a new motor would take a long time and therefore another 3557KO12CR motor was acquired from a non working pipe inspection robot which had a different gear head attached to it. This was a problem because the gear head was too big and could not fit into the current space that is available and therefore the gear heads had to be swapped. Upon closer examination of the gear heads it was noticed that a planetary gear head system is used to transfer torque from the motor to the main gear. This gear system is a good choice because it is able to handle high torque and the space inside the robot is limited, but it is less efficient, more expensive and noisier compared to the spur-type.

\subsection{Gears \& Chain Drive}

The advantage of using gears and chains in the transmission of rotational forces from one device to another is that the forces can be transmitted between devices at different speeds and torques. Inside the body of the robot there are two different sets of rotating gears and one drive chain. The first set of rotating spur gears are used to rotate the camera head along the $X$ axis. The second set is used to rotate the rear wheels using a bevel gear configuration. The drive chain is used to transmit power from the back wheels to the front so that all four wheels are powered. The front camera head contains one set of spur gears which is used to rotate the camera head along the $\mathrm{Y}$ axis.

\subsubsection{Gears}

There are four different sized gears that are used to rotate the camera head along the $\mathrm{X}$ axis. The first gear head which is attached to the motor has 25 teeth, the second has 20, the third has 60 and the fourth has 37, with the gears meshing in that order as shown in Figure 3.7.

The third gear is directly connected to the rotating drive shaft which rotates the camera head. The fourth gear is attached to the front of the third gear with a single screw. The fourth gear transmits torque to the encoder gear which generates voltage pulses as discussed in Section 3.5 . 


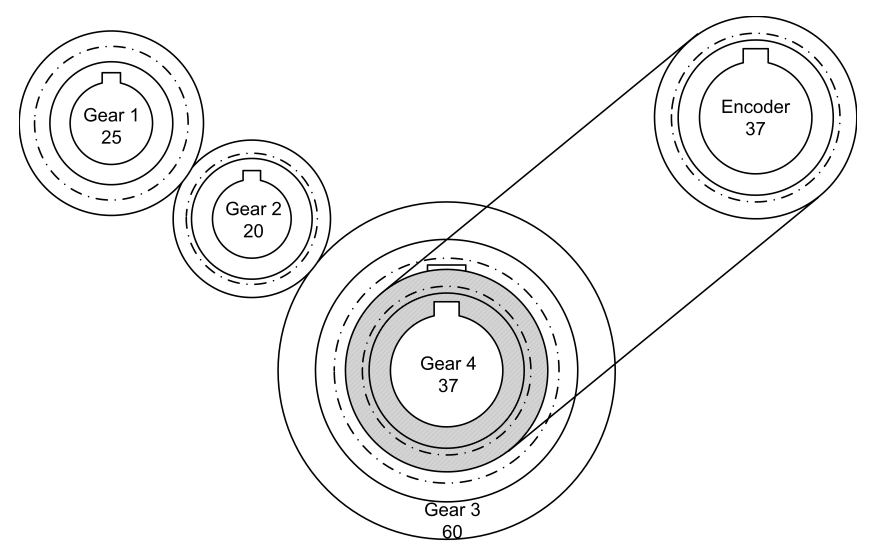

Figure 3.7: Motor Encoder Configuration

The gear train configuration is used to transfer torque $(\tau)$ and angular velocity $(\omega)$ from the first gear to the third gear. Knowing the number of teeth $(\mathrm{N})$ on each gear head the gear ratio can be calculated. The equation relating the torque and angular velocity for a series of 3 gears is shown in Equation 3.1 and 3.2 .

$$
\begin{array}{cc}
\omega_{1} N 1=\omega_{2} N 2=\omega_{3} N 3 & \omega_{1} \tau_{1}=\omega_{2} \tau_{2}=\omega_{3} \tau_{3} \\
\omega_{3}=\omega_{1} \frac{N 1}{N 2} \frac{N 2}{N 3} & \tau_{3}=\tau_{1} \frac{N 2}{N 1} \frac{N 3}{N 2}
\end{array}
$$

When gear values are substituted, Equation 3.3 is produced. The torque is increased by a factor of $\frac{12}{5}$ and the angular velocity has been decreased by $\frac{5}{12}$.

$$
\omega_{3}=\omega_{1} \frac{5}{12} \quad \tau_{3}=\tau_{1} \frac{12}{5}
$$

The second set of spur gears located inside the camera head are used to rotate the camera head along the $\mathrm{Y}$ axis. The same gear train configuration inside the body of the robot is used to transfer angular velocity and torque from the motor to the camera head. The first gear has 80 teeth which is then meshed with the second gear with 100 teeth which then meshes with the third gear which has 90 teeth. The overall gear configuration can be seen in Figure 3.8 


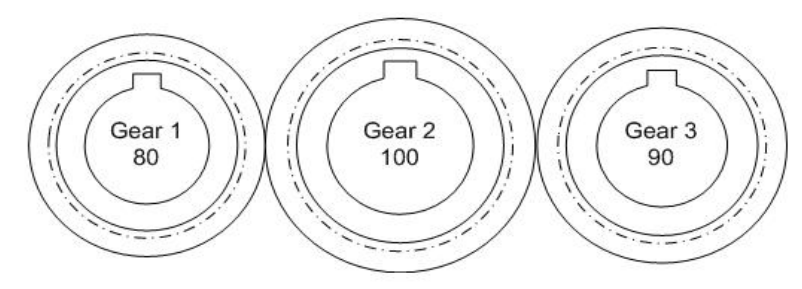

Figure 3.8: Camera Head Y Axis Configuration

Substituting those values into Equation 3.2 the angular velocity is decreased by a factor of $\frac{8}{9}$ and the torque is increased by $\frac{9}{8}$ as shown in Equation 3.4 .

$$
\omega_{3}=\omega_{1} \frac{8}{9} \quad \tau_{3}=\tau_{1} \frac{9}{8}
$$

The set of gears located inside the camera head do not have a major increase in torque or decrease in angular velocity compared to the previous set of gears. This is because the total load being rotated along the $Y$ axis includes the camera and casing, but the $\mathrm{X}$ axis load is higher due to the camera, casing and the rotating shaft.

The Bevel gear configuration is useful in changing the rotation direction of a shaft and therefore it is used to transfer energy from the main motor to the back wheels of the robot. Transferring the energy from the back wheels to the front wheels is achieved using a chain drive discussed in Section 3.4.2.

The smaller gear head containing 15 teeth is attached to the motor which rotates and transfers energy to a second gear head which has 30 teeth. Using Equation 3.2 it is calculated that the torque is increased by a factor of 2 and rotational velocity is decreased by $\frac{1}{2}$ as shown in Equation 3.5 ,

$$
\omega_{2}=\omega_{1} \frac{15}{30} \quad \tau_{2}=\tau_{1} \frac{30}{15}
$$




\subsubsection{Chain Drive}

The chain drive and timing belts are positive drive mechanisms. The chain drives are constructed out of metal which makes them very robust and strong, but at the same time they are heavy and the energy needed to move the whole system is increased.

The chain drive located inside the robot has 95 links and it is used to transfer energy from the rear wheels to the front wheels. The back and front sprockets have 14 teeth each and are attached to the drive shafts. There is a adjustable metal pivot located in the middle of the chain drive set up which is used to increase or decrease the tension in the chain. The pivot can be completely removed if the chain needed to be replaced. The sprockets mesh with the chain, transfering the energy from the back wheels to the front as shown in Figure 3.9 .

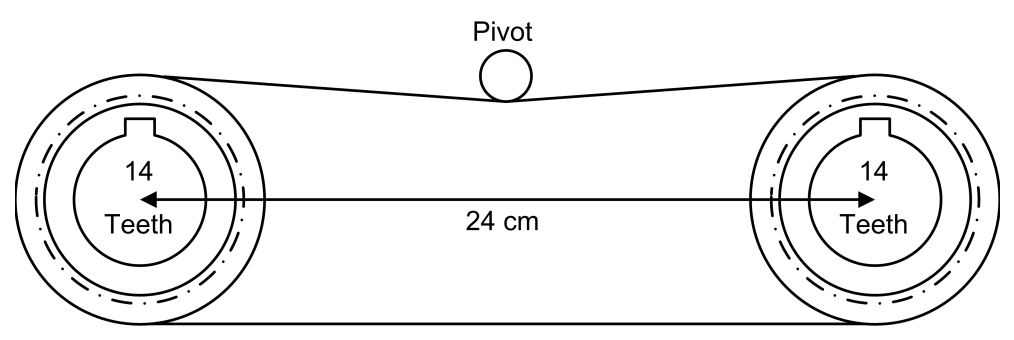

Figure 3.9: Chain Drive Configuration

\subsection{Motor Encoders}

The motor encoder used to measure the number of rotations along the $X$ axis is 2RM360 (Figure 3.10) which is manufactured by SCANCON.

The motor encoder is attached to a fourth 37 tooth gear using a plastic timing belt as shown in Figure 3.7. The encoder produces 360 pulses per revolution and the required operating voltage is between 5 and $24 \mathrm{~V}$ DC. In total there are three output signals (A, B \& Z). The A and B signals are used to count the number of pulses rotated by the encoder and the $Z$ signal is set high if a full 360 degree rotation has been completed. The A and $\mathrm{B}$ signals are also used calculate the direction the encoder is rotating 


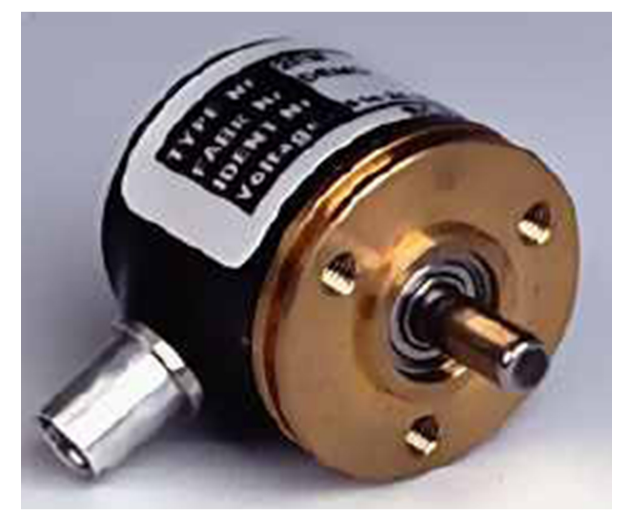

Figure 3.10: 2RM360 Encoder

in. Figure 3.11 demonstrates how a clockwise $(\mathrm{CW})$ rotation outputs a sequence for $\mathrm{A}$ and $\mathrm{B}$ signals. An anti-clockwise (ACW) rotation produces a reversed sequence.

\footnotetext{
Rotation: Clockwise (CW) from shaftside

(inv $=$ inverted channels)
}

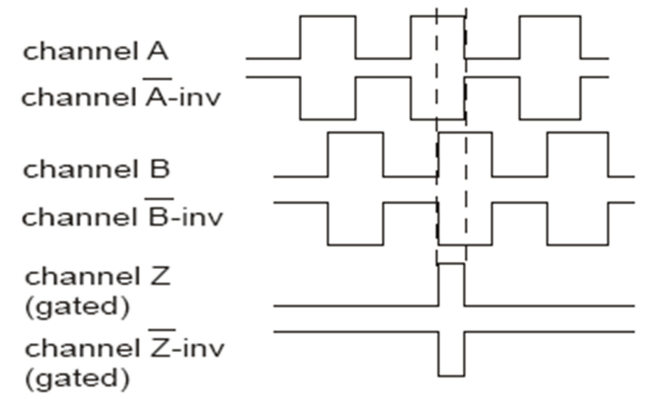

Figure 3.11: 2RM360 Output Signals

A second motor encoder was installed in the original robot design. This motor encoder is constructed using a toggle switch and a small screw which is attached to the end of a rotating shaft shown in Figure 3.12

When the rotating shaft completes a single rotation the screw comes into contact with the switch and a high pulse is generated. This second motor encoder is not utilised in the robot redesign due to 2RM360 having a higher resolution and therefore it is made redundant. 


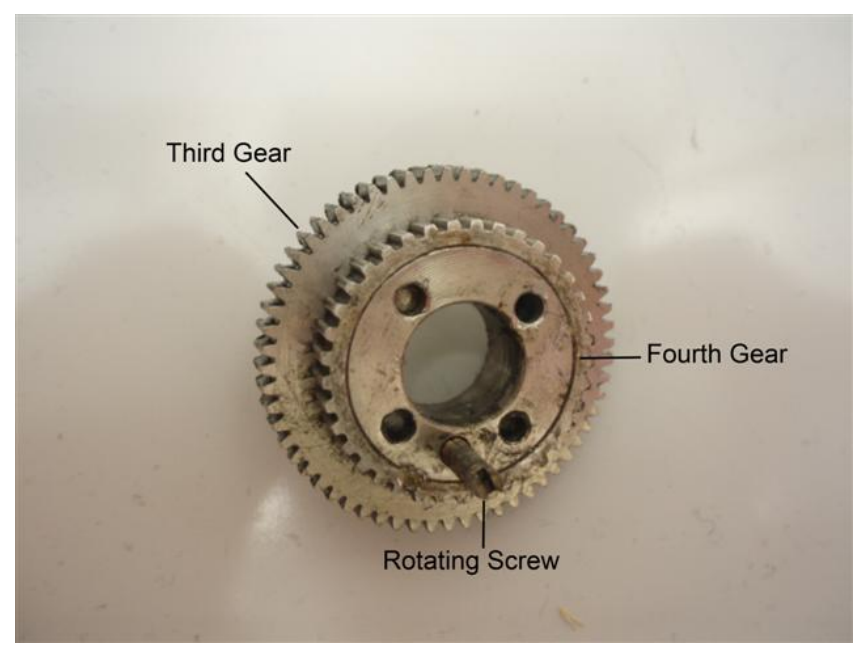

Figure 3.12: Second Motor Encoder Configuration

The third motor encoder is used to count the number of revolutions along the $\mathrm{Y}$ axis. The encoder is constructed by attaching a small metal brush and two metal contacts at the bottom of the second gear. The encoder configuration can be seen in Figure 3.13 .
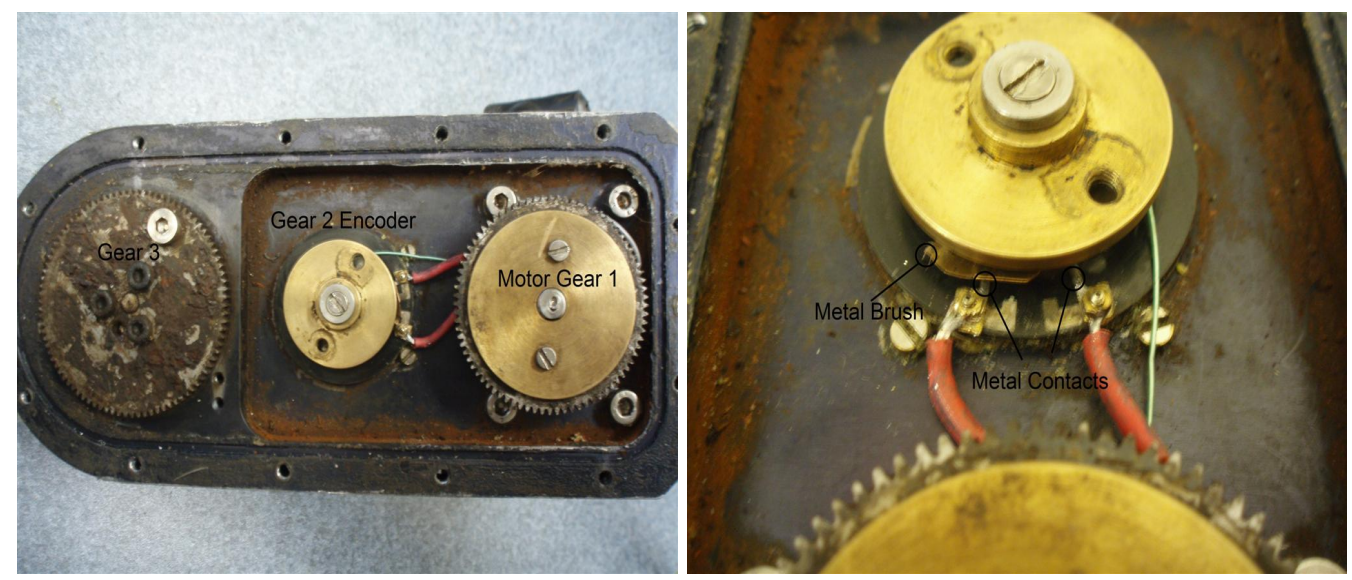

Figure 3.13: Camera Head Y Axis Gear Configuration

Each time the gear head rotates, the metal brush will come in contact with one of the metal plates, generating a high pulse. By counting the number of high pulses, the micro-controller can calculate how far the camera has rotated. The actual encoder design is not very reliable due 
to the metal brush not generating a consistent electrical contact. This is a problem which could result in some pulses being missed by the microcontroller, resulting in damage being caused to the camera head by coming into contact with the external casing. One possible solution would be to completely ignore the $\mathrm{X}$ axis encoder design and only allow the camera head to rotate along the $\mathrm{Y}$ axis. The second solution would be to calibrate the micro-controller to rotate the motor in a particular direction for a length of time and then rotate the camera for the same length of time back to the starting point.

The first solution suggested is not desirable due to the need of having the ability to rotate on both axes and therefore the second option would have to be implemented. The optimal solution would be to replace the whole encoder with a new, more reliable one but because of the time factor the solution is implemented by software which will keep track of the time that the motor turns as discussed in Section 5, At the same time the encoder is configured to send a high pulse if a full rotation is complete and the timer is implemented just in case the encoder fails to send a high pulse. 


\section{Chapter 4}

\section{Hardware}

\subsection{Power Source}

The power source used inside the robot is a Mini V300B12C250BL DC to DC converter purchased from Vicor Power. The specifications of the converter are discussed in Section 2.3.3 and the pinout of the converter is shown in Figure 4.1 .

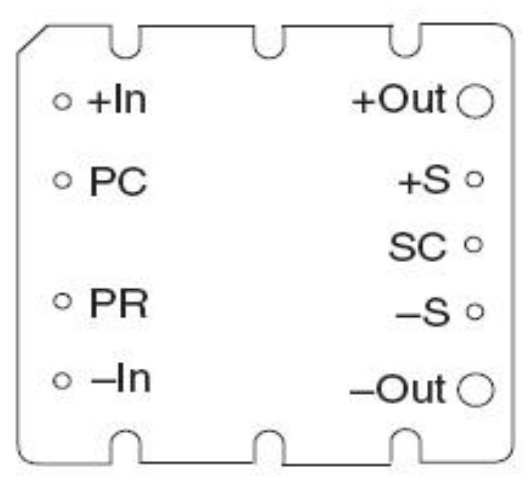

Figure 4.1: V300B12C250BL DC to DC Converter

The -In and +In pins connect to the main voltage of $240 \mathrm{~V}$ DC. The module contains internal "watchdog" circuitry which monitors input voltage, operating temperature and internal operating parameters. In the event that any of these parameters are outside of their allowable operating range, e.g, temperature greater than $100^{\circ} \mathrm{C}$, the module will shut down and the 
PC pin will go low. The PR pin is used for parallel operation to increase the amount of current that can be delivered on the \pm Out pins. To achieve this the modules must have the same input voltage, output voltage and power level. The module has two interface architectures which include DC coupled and AC coupled single-wire shown in Figure 4.2 .
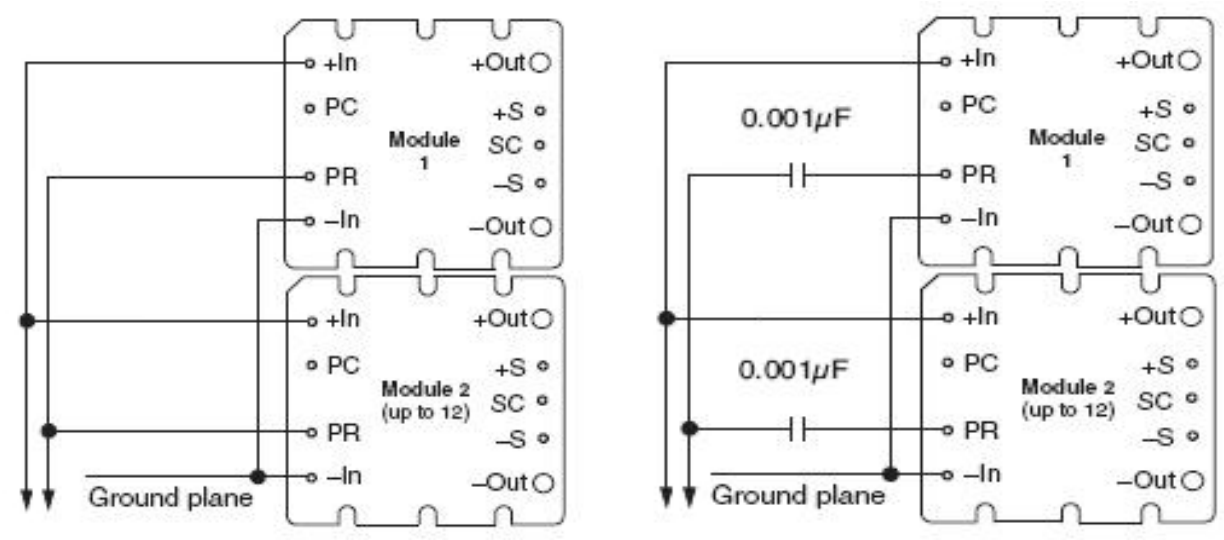

Figure 4.2: Left: DC Coupled Interface Right: AC Coupled Interface

The +Out and -Out pins output $12 \mathrm{~V}$ DC for the operating circuitry. The output voltage of the module can be adjusted via external trim down and trim up resistors. The trim down resistor is used to connect the SC, $-\mathrm{S}$ and -Out pins in a series connection. This circuitry configuration is used if the output voltage needs to be lower than $12 \mathrm{~V}$. The trim up resistor connects $\mathrm{SC},+\mathrm{S}$ and +Out pins in a series connection if the output voltage needs to be higher than $12 \mathrm{~V}$. Both circuit configurations can be seen in Figure 4.3 Equations 4.1 and 4.2 show how to calculate the value of the trim up, $R_{U}$, or trim down, $R_{D}$, resistors for a required output voltage $V_{\text {out }}$, where $V_{\text {nom }}$ is the nominal output voltage of $12 \mathrm{~V}$. Special care is needed when the trim up resistor is used to make sure that the maximum rated power is not exceeded.

$$
\begin{gathered}
R_{D}(\Omega)=\frac{1000 \times V_{\text {out }}}{V_{\text {nom }}-V_{\text {out }}} \\
R_{U}(\Omega)=\frac{1000\left(V_{\text {out }}-1.23\right) V_{\text {nom }}}{1.23\left(V_{\text {out }}-V_{\text {nom }}\right)}-1000
\end{gathered}
$$




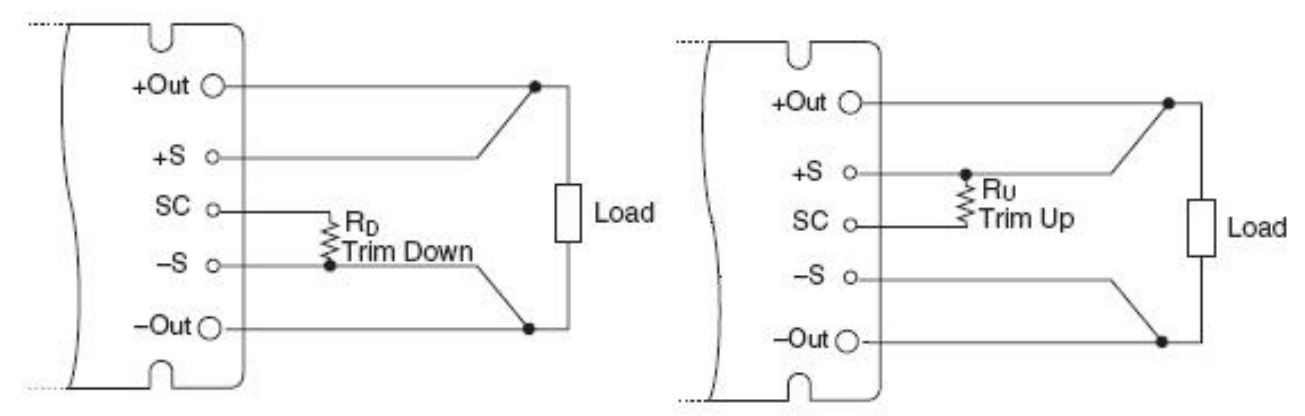

Figure 4.3: Left: Trim Down Right: Trim Up

The configuration of the DC to DC converter does not use trim up or down resistors because the robot electronics require a $12 \mathrm{~V}$ DC power source. To eliminate any short circuit that might occur all of the terminals are covered in glue which prevents any loose wire coming into contact with the terminals. A $2.2 \mu$ F bypass capacitor is inserted between the + In and -In terminals to reduce noise along the power lines. The module is attached to a brass top plate with four screws as shown in Figure 4.4 and before attaching the plates together a heat sinking compound is inserted between the surfaces.

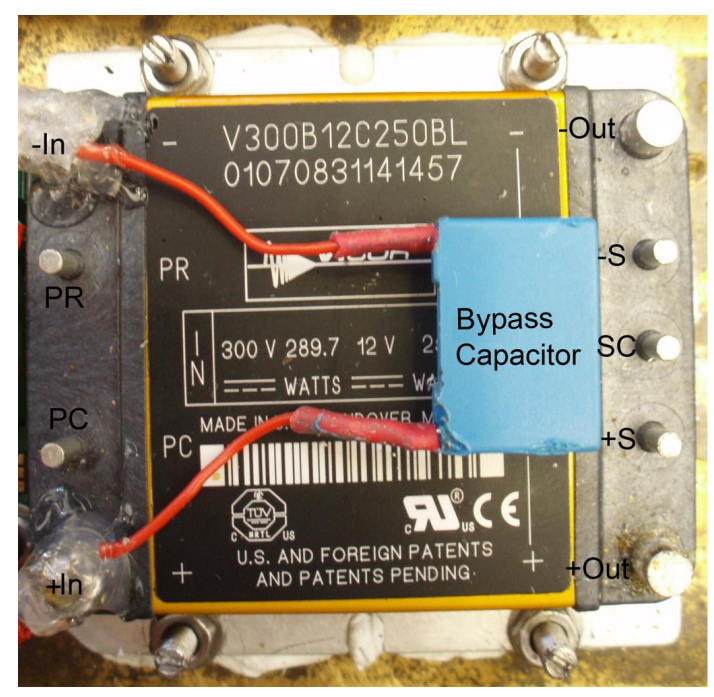

Figure 4.4: DC to DC Converter Pin Configuration 


\subsection{Motor Controller Board}

The motor controller board contains two motor drivers, a micro-controller, $5 \mathrm{~V}$ regulator, RS-485 and RS-232 transceivers. The micro-controller employed is an AT89C51AC3 which has the ability to produce five Pulse Width Modulated (PWM) outputs, UART or Serial Peripheral Interface (SPI) communication and can record any external interrupts using a Programmable Counter Array (PCA). The RS-485 transceiver is used to send and receive data from the base station using a 180 metre cable. The motor drivers are a Sabertooth 5 A dual output supplied by Dimension Engineering.

\subsubsection{Sabertooth 5 A Dual Motor Driver}

The motor driver boards chosen to control the motors are Sabertooth Dual 5 A shown in Figure 4.5 [22]. The main reason for choosing this motor driver is the ability to drive two independent motors with two independent PWM signals. Nominal input voltage is between 6 and $18 \mathrm{~V}$ DC and
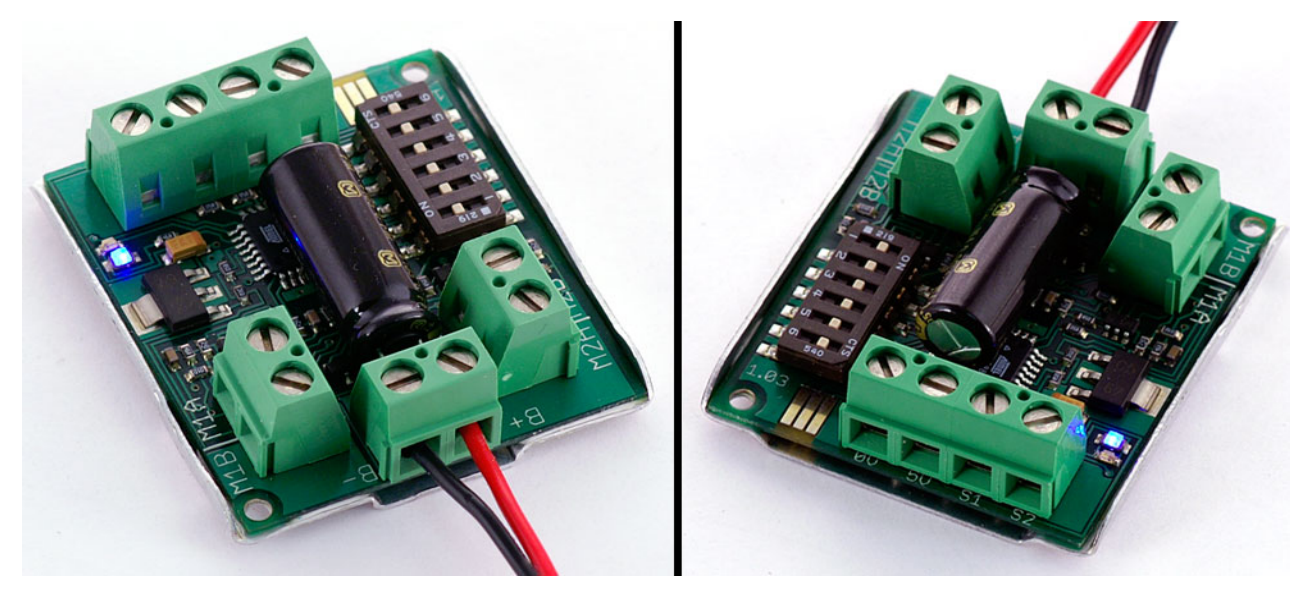

Figure 4.5: Sabertooth 5 A Dual Motor Driver

the absolute maximum is $20 \mathrm{~V}$ DC. Each channel can handle 5 A continuous current and a peak current of $10 \mathrm{~A}$. The dimensions of a single board are $40 \times 45 \times 13 \mathrm{~mm}$ and weighs $19 \mathrm{~g}$.

The motor drivers have different operating modes which include analogue input, R/C micro-controller, simplified serial and packetised serial. 
Each one of the modes can be selected by using six switches located on the motor driver board. The first two switches select the mode required and the last four switches select the different operations that the mode can achieve.

The analogue input mode was not implemented due to the need of physical interaction between the user and the variable resistor, which is not possible due to the robot being inside a pipe. The simplified and packetised serial were considered for implementation but were discarded due to the limited number of communication lines available along the connection cable between the robot and the base station as discussed in Section 3.2.1. The best approach is to use the $\mathrm{R} / \mathrm{C}$ micro-controller mode which receives a PWM signal from the micro-controller to control the speed and direction of the motor. The advantage of using this mode is the ability to program the micro-controller to receive user commands and change the pulse width of the PWM signal accordingly.

To enable operation on the motor driver using PWM signals an R/C micro-controller mode is selected by setting switch 1 to low, 2 to high and 6 to low. The other three switches can enable or disable the mixing mode, exponential response and a lithium cut off point. If the mixing mode switch is set to high, two motors can be controlled with one PWM signal. This switch must be set low because all of the motors are independent and each PWM signal will control one motor. If the robot required a fast turning speed and a fast stop response the exponential response switch would be set to low, but because the robot is relatively slow the switch is set in a high state resulting in a linear response. The lithium cut off switch is set low if the power source uses lithium cells. This is done to prevent any damage to lithium batteries. The power source used is a DC to DC converter and therefore the switch is set to high.

The motors used to control the camera head share the same motor driver board. The motor used to control the camera head along the $\mathrm{X}$ axis is attached to M1+ and M1-, whilst the Y axis motor is attached to the M2+ and M2- terminals as shown in Figure 4.6. The PWM signals used to control the motors are attached to the S1 and S2 connectors respectively. The power source for the motors is attached to the main board via the $\mathrm{B}+$ and B- terminals. The main motor is attached to a second motor driver board 
with the same configuration as the previous motor driver board.

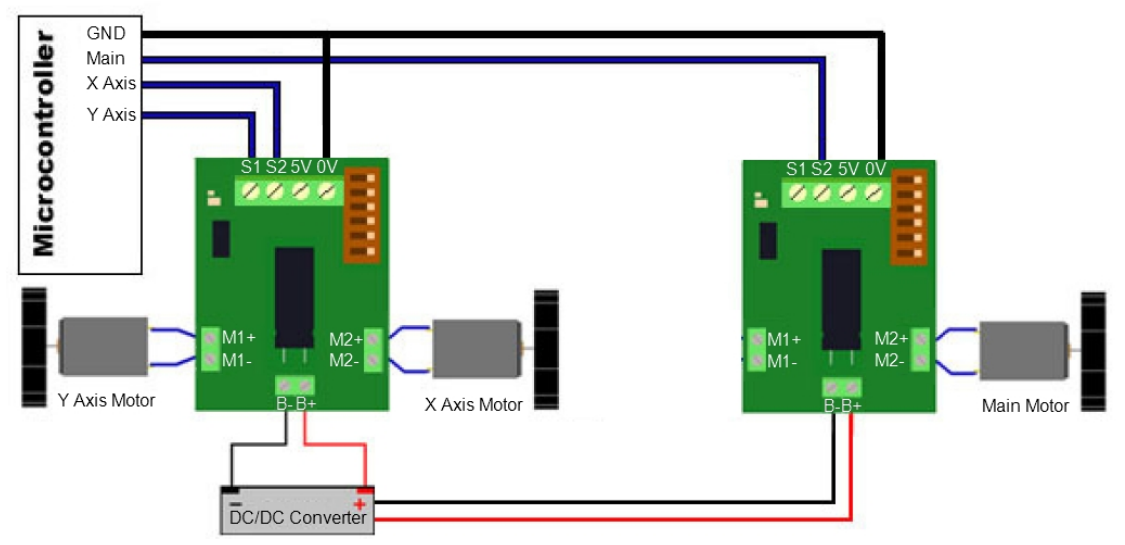

Figure 4.6: Sabertooth Motor Configuration

To achieve forwards and backwards movement on a single motor the PWM signal width has to be between $1 \mathrm{~ms}$ and $2 \mathrm{~ms}$. A pulse width of $1 \mathrm{~ms}$ would result in full speed in a forward direction of the motor and a 2 ms pulse would result in full speed in the opposite direction. The motor would stop if the pulse width is $1.5 \mathrm{~ms}$ as shown in Figure 4.7. To avoid any damage to the motor drivers, a steady increase in the pulse width is needed and any sudden large changes should be avoided at all times.

The motor drivers have built in status and error LEDs. The blue LED will be turned on if the motor driver is in a working condition with no errors. The red LED will turn on if the motor driver has detected an internal problem and has shut down due to overheating or the maximum current being exceeded. To prevent exceeding the maximum current, two $2.5 \mathrm{~A}$ resettable fuses and two zener diodes are inserted as shown in Figure 4.8 .

If the applied voltage exceeds $12 \mathrm{~V}$ the zener diode will break down and a current path made to ground. The excess current will blow the fuses and both of the motor drivers will turn off. To turn on the motor drivers the micro-controller sends a high signal from pin 2 port 2 to the base of the transistor. Turning the transistor on allows the current to pass through, turning on the relay switch which connects the motor drivers to the $12 \mathrm{~V}$ power supply. The voltage comparator LM339AD is used to check if the $12 \mathrm{~V}$ reference voltage matches the voltage of the motors. If the voltages 

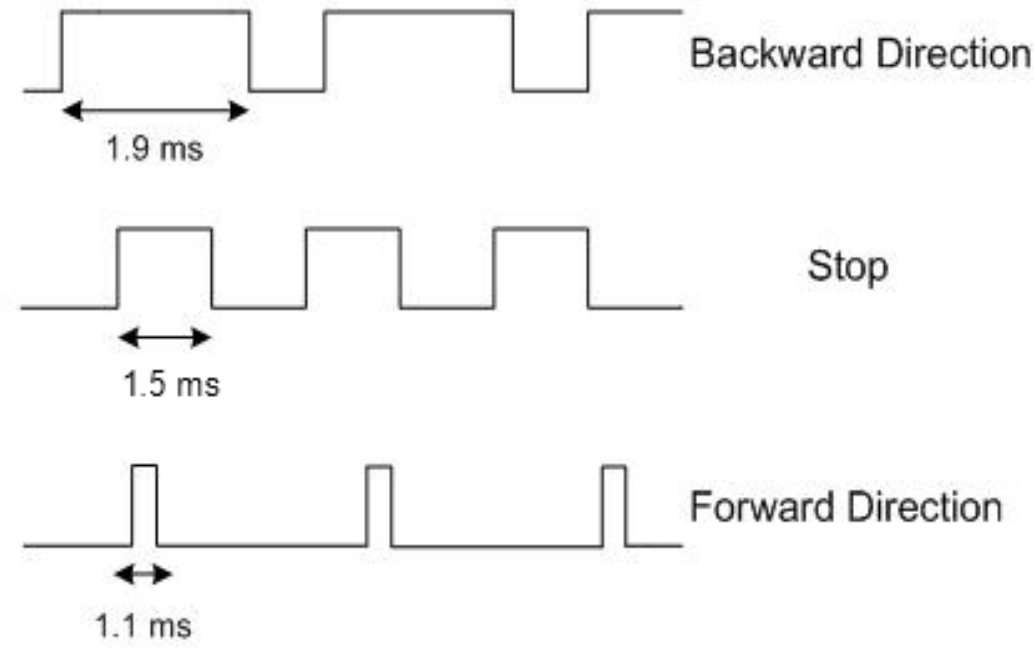

Figure 4.7: Motor PWM Signals

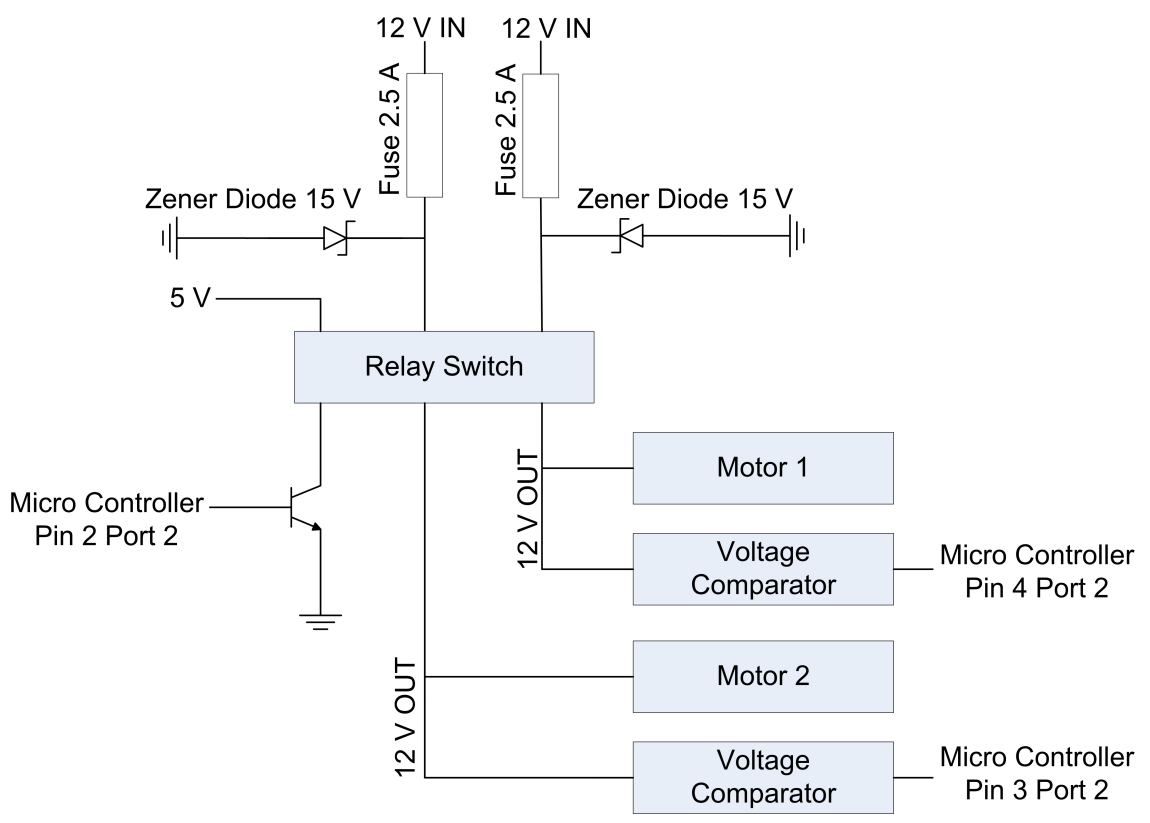

Figure 4.8: Motor Circuit Protection 
do not match, a low signal is sent to the micro-controller to shut down the motor drivers and notify the user about the problem. The micro-controller code implemented to turn the motor drivers on and off is discussed in Section 5.3.1. To prevent over heating, the motor drivers are attached to a top plate of the chassis using four screws as shown in Figure 4.9. Before the motor drivers were attached to the brass chassis a heat sinking compound was added between the plates.

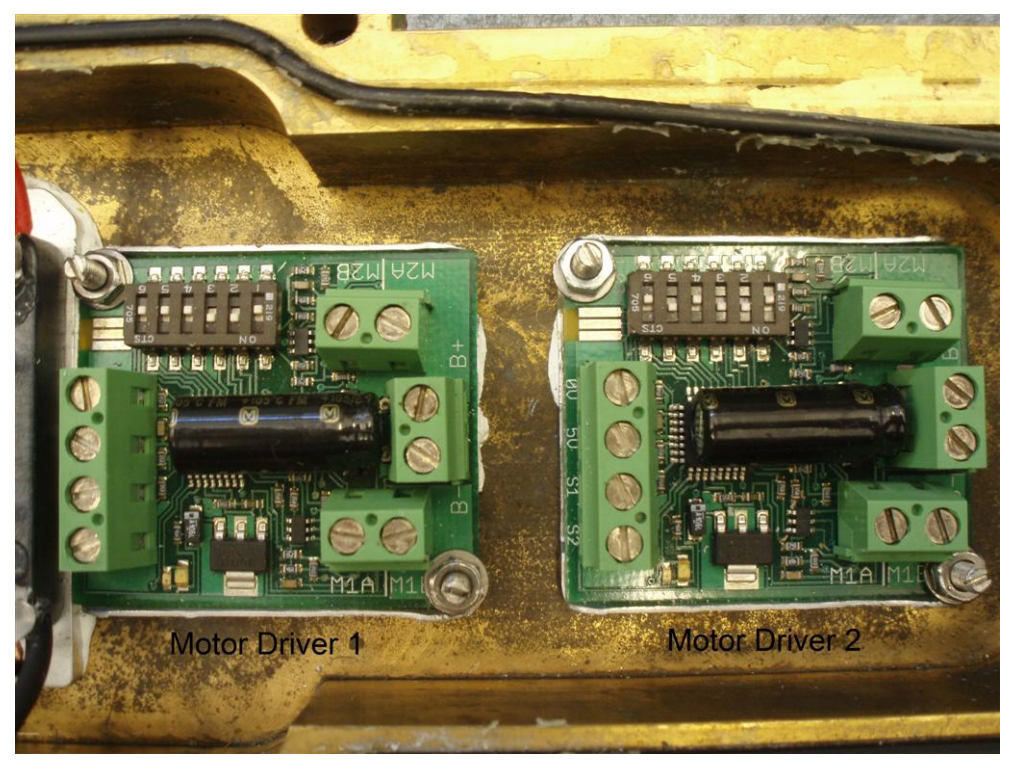

Figure 4.9: Motor Drivers $1 \& 2$

\subsubsection{Micro-controller AT89C51AC3}

The AT89C51AC3 micro-controller is a 8-bit, 80C51 core architecture with $64 \mathrm{~KB}$ of flash memory. The micro-controller can be powered by either a $5 \mathrm{~V}$ or a $3.3 \mathrm{~V}$ regulator, the former being arbitrarily selected. The Programmable Counter Array (PCA) module provides more timing capabilities with less CPU intervention than the standard timers/counters. Its advantages include reduced software overhead and improved accuracy. The PCA pins can be configured either as a PWM signal output or as an external interrupt input. The external interrupt method is used to trigger an event inside the micro-controller each time a high or a low pulse is detected. This feature is used to calculate the current position of the camera 
head using the motor encoders. The $20 \mathrm{MHz}$ crystal oscillator provides a high accuracy PCA clock which results in accurate PWM signals and external interrupts with an error margin of $0.0005 \%$. The PWM signal output is designed to have a minimum width of $1 \mathrm{~ms}$ and a maximum of $2 \mathrm{~ms}$ as discussed in Section 4.2.1,

The serial communication between the micro-controller and the base station uses receive (RX) and transmit (TX) lines located on port 3. These lines are used to communicate via the RS-485 or SP3238 (Section 4.2.4) communication chips. The RS-485 chip is used as a communication device between the micro-controller and the base station when the robot is out in the field and the distance exceeds $15 \mathrm{~m}$. The SP3238 chip is used for the communication when the robot is being serviced and it has to be reprogrammed. This design approach allows the robot to be flexible if any future developments were to be added or removed. For this reason external connectors are added to port 1 and 4 to make them easily accessible and therefore removing the need for a new redesign of the main board.

The initial design had the micro-controller connected to both communication devices at the same time. This design approach introduced a bus contention problem, as the RS-485 chip would pull the line high but at the same time the SP3238 chip would pull it low. This would result in no communication being established between the home base and the microcontroller. To avoid this problem a double toggle switch has been inserted as shown in Figure 4.10 .

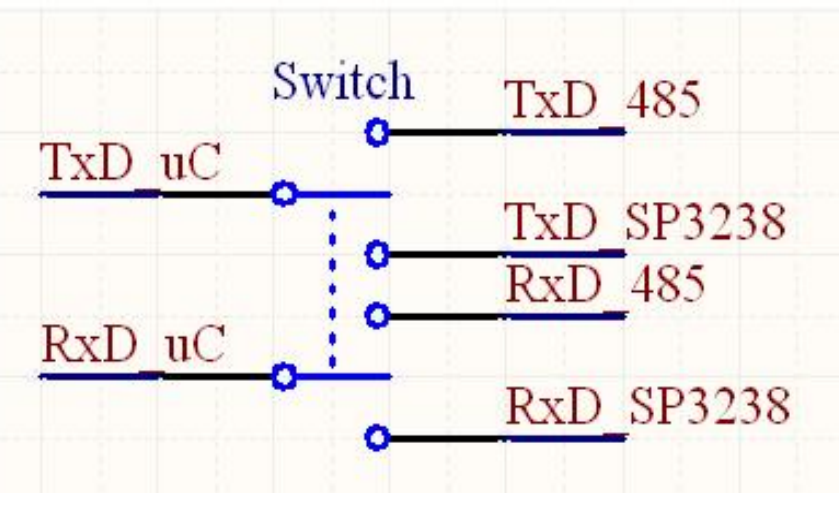

Figure 4.10: RX and TX Double Switch 
When the switch is selected in the RS-485 direction the RX and TX lines latch on to the RS-485 chip, and in the opposite direction the microcontroller connects to the SP3238. The software used for the communication by the micro-controller is discussed in Section 5.1 .

\subsubsection{Main Board RS-485 Transceiver}

The RS-485 LTC1387 chip transceiver is chosen because of the capability to drive a signal over $1200 \mathrm{~m}$ without any data loss. The reason for choosing this chip is the capability of having either two RS-232 or one RS-485 transceiver and being able to communicate between multiple transceivers using the same RX and TX lines. Having the ability to communicate between multiple transceivers eliminates the need for extra data lines between the camera board and the base station board. The transceiver operates from a single $5 \mathrm{~V}$ supply regulator and has a low supply current of $7 \mathrm{~mA}$.

The drivers and receivers on the LTC1387 chip can be controlled independently. This is achieved by the micro-controller changing the receive (RXEN) and transmit (DXEN) enable lines to either a high or a low. If the device has to be turned off at any time the micro-controller sends a low signal to the control $(\mathrm{ON})$ pin. The pin configuration of the chip can be seen in Figure 4.11 .

To establish a connection between two RS-485 transceivers, both receive $(\mathrm{A} \& \mathrm{~B})$ and transmit $(\mathrm{Y} \& \mathrm{Z}$ ) terminals have to be connected to each other and therefore extra care is needed when setting up the main, camera and base station boards. This is due to the differential set up of the receiver and transceiver. The main, camera and the base station boards would receive the same data that they were trying to transmit. This problem is solved by hardware discussed in Section 4.4 .2 and by software in Section 5.1.5.

\subsubsection{SP3238 Transceiver}

The initial connection between the micro-controller and the computer is made using a SP3238 RS-232 transceiver. The chip is powered by a $5 \mathrm{~V}$ 


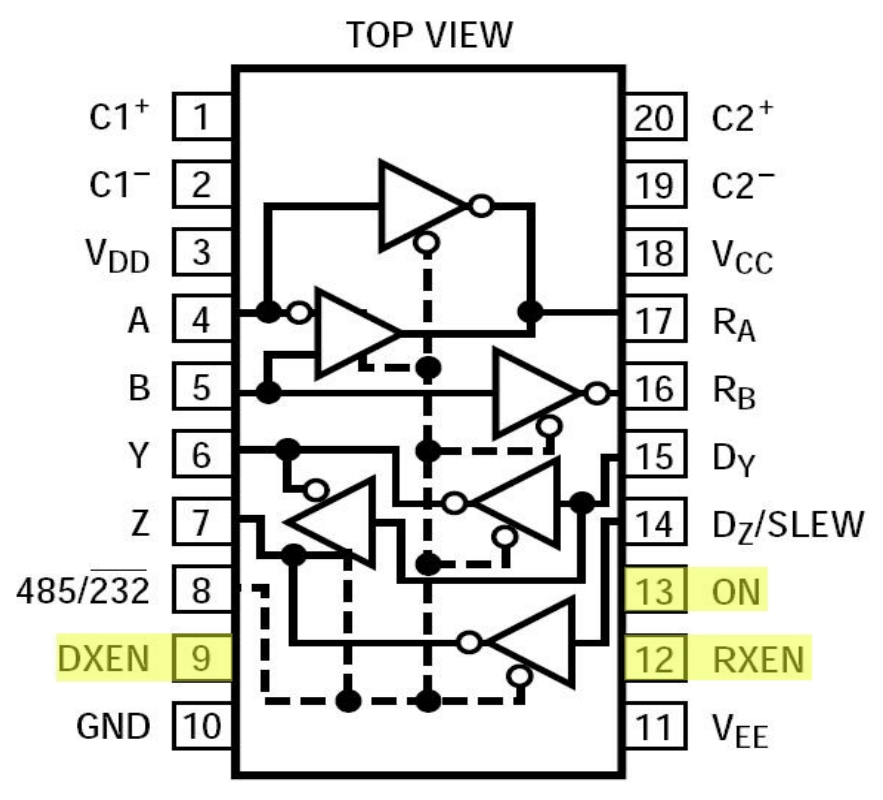

Figure 4.11: LTC1387 Pin Configuration

regulator though $3 \mathrm{~V}$ operation is also possible. The chip comes in a 28 pin package and internally there are 3 receivers and 5 transmitters.

The programming of the micro-controller required the program enable and reset switches to be pressed as described in Section 4.2.2. To eliminate this step, a circuit has been designed which uses the Request To Send (RTS) and Data Terminal Ready (DTR) data lines. Using these lines along with RX and TX, the computer can connect to the micro-controller automatically with no user interaction.

\subsection{Camera EVI-370 \& Camera Board}

At the start of the project a new camera was going to be purchased and integrated, but the supply of a single camera was difficult to obtain as discussed in Section 2.3.5, and therefore the existing SONY EVI-370 camera was employed. The specifications of the camera are listed in Section 2.2.3.

The board located inside the camera head measures $34 \times 61 \mathrm{~mm}$. The board has been designed to be small in size due to the room constraint inside the camera head. The board contains $8 \mathrm{~V}$ and $5 \mathrm{~V}$ regulators along 
with RS-485 and MAX202 transceiver chips. The $8 \mathrm{~V}$ regulator is used to power the camera and the $5 \mathrm{~V}$ regulator provides power to the RS- 485 and MAX202 transceivers. The video signal received from the camera is transmitted to a coax cable to conserve image quality.

\subsubsection{Camera EVI-370}

The features of the camera are listed in the Section 2.2.3. The camera uses a VISCA control architecture which has been designed to interface to a wide variety of video equipment with a computer. When using VISCA, up to 7 cameras can be connected to a single RS-232 communication device. Located inside the camera is a MAX232 transceiver which is to establish communication between the camera and the computer. The communication is established using a RS-232 connector, setting the baud rate to 9600 and selecting a 8-bit data format, 1 start bit, 1 stop bit and no parity.

The on screen display allows GEN-lock, date and time, and camera ID to be overlayed on the video image. The only display that is needed is time and date, which will help when images are saved and later analysed. The different command lines used to control the camera are discussed in Section 5.1.1.

\subsubsection{Camera Board}

The data packets that are sent from the base station to the camera board are in RS-485 differential signal level format. The RS-485 transceiver located on the camera board converts the incoming differential signals to TTL logic. A problem occurs inside the camera because there is a MAX232 transceiver which requires RS-232 signal levels. To solve this problem a MAX202 chip is added to the camera board which has a TTL logic input and a RS-232 signal output. The overall configuration between the base station and the camera is shown in Figure 4.12 .

The current drawn by the camera is $0.4 \mathrm{~A}$ with camera zoom motors off and $0.48 \mathrm{~A}$ with motors active. To prevent any damage to the on-board circuits some protection has been implemented. The circuit has been constructed using a relay switch and a transistor. The base of the transistor is 


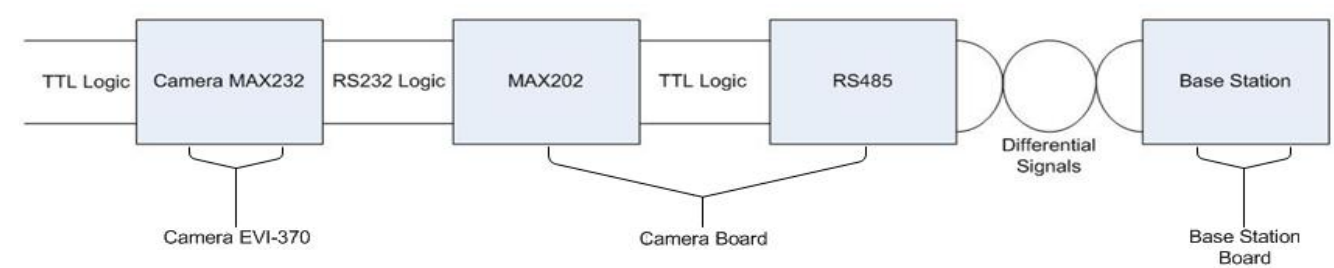

Figure 4.12: Camera Board Setup

connected to pin 1 port 2 of the micro-controller. The user sends a command packet to the micro-controller requesting that the camera be turned on. The micro-controller then sets the pin to high which turns the transistor on and allows current to pass through the relay switch. The relay switch then applies positive voltage to the camera as shown in Figure 4.13 which is similar to the circuit shown in Figure 4.8 .

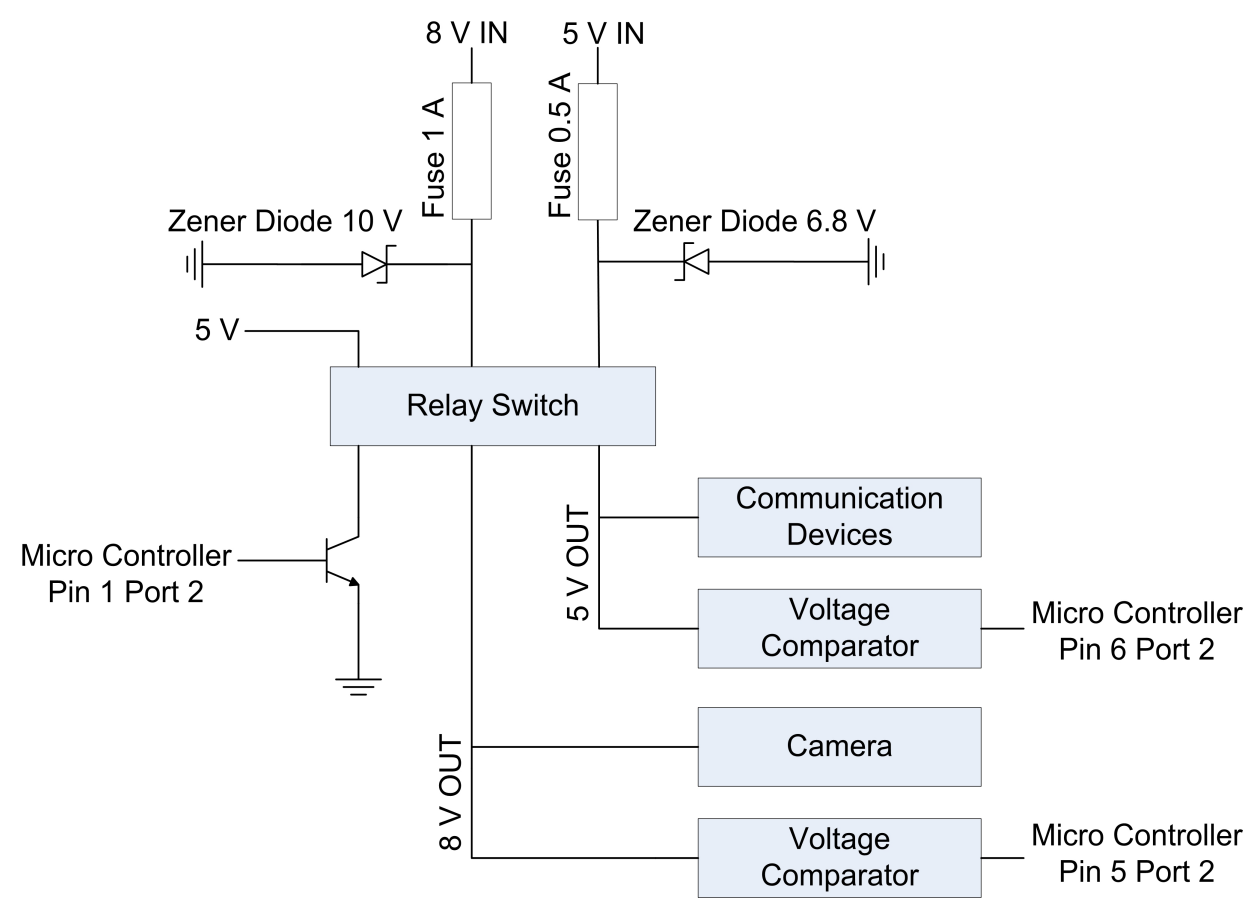

Figure 4.13: Camera Circuit Protection

To prevent any damage from excess voltage, resettable fuses and zener diodes are inserted similar to the earlier circuit described in Section 4.2.1. 
The voltage comparator is used to compare voltage levels between the $8 \mathrm{~V}$ and $5 \mathrm{~V}$ reference voltage with the power levels of the camera and communication devices. The output signal of the voltage comparator is sent to pin 5 port 2 of the micro-controller and the output of the second comparator is sent to pin 6 port 2. If the voltage levels of the camera and communication devices do not match with the reference voltage levels the comparator outputs a low signal to the micro-controller. A low signal means there is a problem inside the circuit and the micro-controller shuts down this part of the circuit to avoid damage and notifies the user about the problem. The micro-controller code that is implemented to turn the camera board on and off is discussed in Section 5.3.1

\subsubsection{Light Emitting Diodes}

The environment that the robot operates in is dark and therefore an illumination source is required. The light bulbs used in the previous robot drew an excessive amount of current. To reduce this current draw, light emitting diodes (LEDs) were installed. The four LEDs chosen had to provide sufficient illumination, yet be small in size to fit inside the camera head's compartment.

The LEDs chosen were TLCW5100 from Vishay Electronics [23]. The maximum operating voltage $\left(V_{\text {drop }}\right)$ per LED is $4.5 \mathrm{~V} \mathrm{DC}$ and the maximum continuous current $\left(I_{\text {cont }}\right)$ is $30 \mathrm{~mA}$, thus the total current drawn is $120 \mathrm{~mA}$. To avoid damage to the devices from overcurrent, a small resistor was calculated using Equation 4.3 and placed between the $8 \mathrm{~V}$ regulator $\left(V_{\text {reg }}\right)$ and the LED's.

$$
\begin{aligned}
& \text { Resistor }=\frac{V_{\text {reg }}-V_{\text {drop }}}{I_{\text {cont }}} \\
& \text { Resistor }=\frac{8-4.5}{0.03} \\
& \text { Resistor }=116.67 \Omega
\end{aligned}
$$

The resistor placed inside the circuit has a slightly higher value of $120 \Omega$ providing an added margin for safety. 


\subsection{Base Station Board}

The base station board is used to communicate between the computer, main control board and the camera board. The board contains a RS-485 transceiver, a FT-232BQ USB chip and an OR gate. A power source problem was encountered because all of the chips required a $5 \mathrm{~V}$ power supply. This was a problem because out in the field only the main power voltage levels will be available and converting $240 \mathrm{~V}$ AC to $5 \mathrm{~V}$ DC would be impractical. To fix this problem the USB chip has been chosen which can deliver $5 \mathrm{~V}$ DC from the computer and supply a maximum current of $0.1 \mathrm{~A}$. The chips used on the board are low power and this maximum current would not be exceeded.

\subsubsection{FT232BQ USB UART}

The FT232BQ USB chip is used to connect between the computer and the RS-485 chip and it has the following features:

- Single chip USB $\leftrightarrow$ Asynchronous serial data

- Auto transmit buffer for RS-485

- Integrated 3.3 V regulator for USB IO

- Support for high power USB Bus powered devices

The chip uses an external $6 \mathrm{MHz}$ crystal oscillator to generate a high frequency internal clock. This crystal uses a $\times 8$ clock multiplier to generate a $48 \mathrm{MHz}$ reference clock for the USB and Baud Rate Generator blocks. The chip uses USB Data Signal Plus (USBDP) and Minus (USBDM) data lines to establish communication with the computer. The USBDP and US$\mathrm{BDM}$ require external $27 \Omega$ resistors to limit the input current to the chip as shown in Figure 4.14 .

The communication between the chip and the RS-485 transceiver is achieved using RXD and TXD lines. The computer can provide enough current to establish communication and to power two LEDs which are turned on each time the RXD and TXD lines are pulled high. This is a great feature which is used to help debugging during the implementation. 


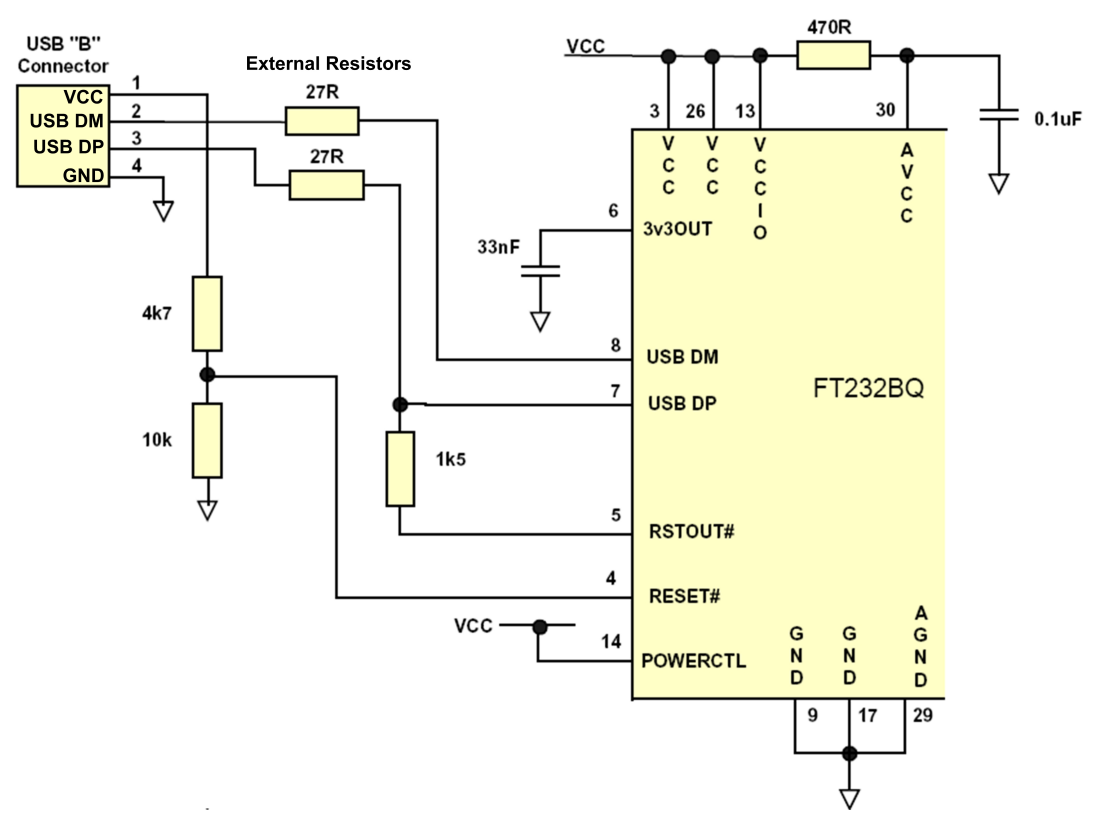

Figure 4.14: FT232BQ Configuration

\subsubsection{Base Station RS-485 Transceiver}

The same LTC1387 RS-485 transceiver is used at the base station as on the motor controller board. Figure 4.15 shows the connection between two LTC1387 chips. To enable communication between the chips the receive and transmit drivers have to be connected together. This is done because the RS-485 transceiver works as a differential driver instead of a single common ground driver. The connected drivers introduce a problem because pin seven is connected to pin five and pin six is connected to pin four. This means when a data packet is transmitted from the base station pins six and seven to the micro-controller pins four and five the same data will be received on the base station pins four and five.

In this section the problem is solved using a hardware configuration as shown in Figure 4.16, but in Section 5.3.1 a similar problem is encountered which can not be solved using hardware and therefore a software approach had to be used.

The problem is solved using the TXDEN output pin on the FT232BQ chip which is set high each time a data packet is transmitted. The OR gate has two inputs which come from the RS-485 receive and TXDEN lines. 


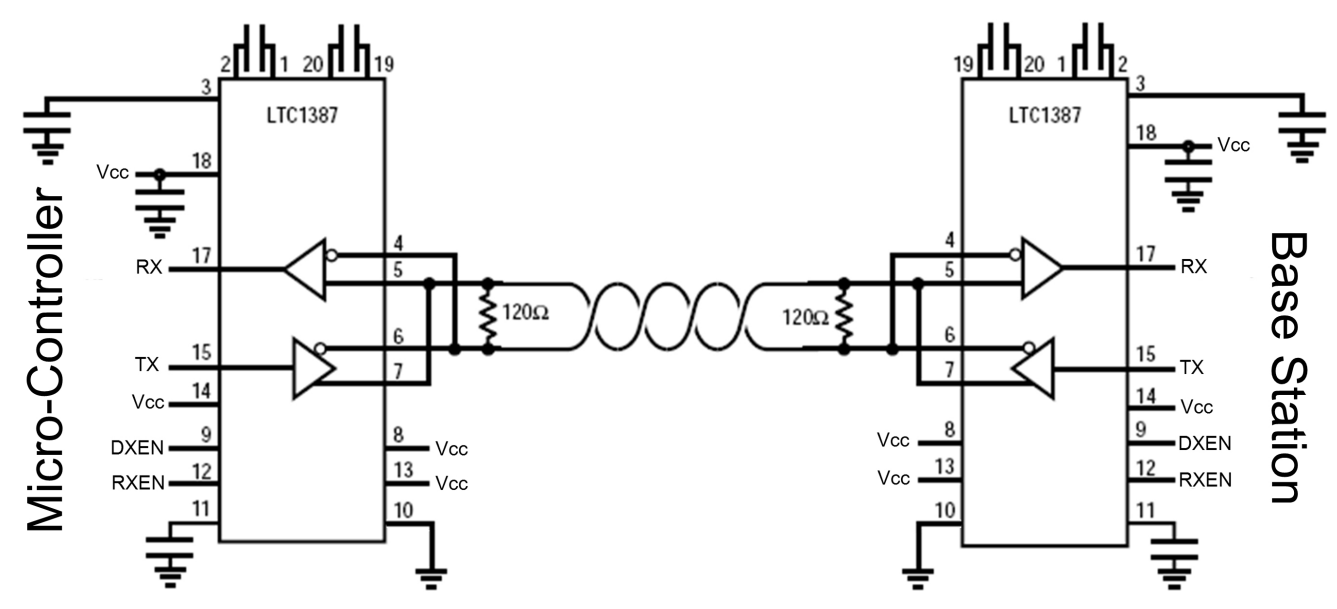

Figure 4.15: RS-485 Configuration

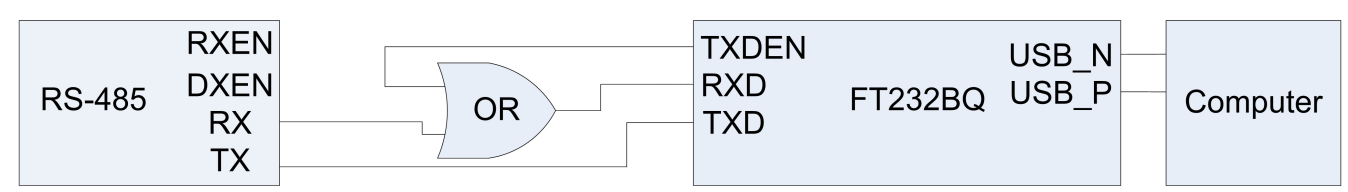

Figure 4.16: FT232BQ and RS-485 Configuration 
The output of the OR gate is connected to the receive line of the FT232BQ chip. When the FT232BQ chip transmits a data packet on the TXD line, the TXDEN signal is pulled high and the output on the OR gate becomes permanently high. The FT232BQ chip will not receive any data on the RXD pin which will remain high regardless of data on the RX line. If data are received on the RS-485 chip this means the TXDEN pin is set low and therefore the data received will be transmitted to the FT232BQ chip. Figure 4.17 shows the TXDEN line pulled high on one input and at the same time data packet reflections being received on the second input. The output of the OR gate never changes and therefore the computer does not receive any returned data signals.

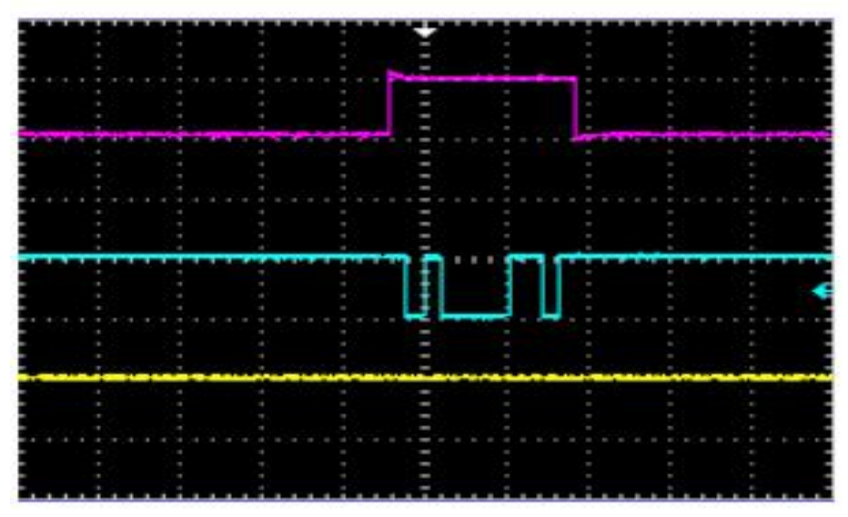

Figure 4.17: Purple: TXEND Blue: RS-485 RX Yellow: FT232BQ RXD

\subsubsection{Video Capture Card}

The video image that is transmitted back to the computer is a composite video signal. This video signal has to be displayed on the remote computer for the user to determine if any problems were encountered inside the pipe. One possibility was to purchase a desktop video capture card which would provide a high resolution image, but it would be impractical because in the operating environment the user would require a light and portable laptop. For this reason the video capture card that was purchased and implemented is a USB pluggable device which results in good portability and image quality. The video capture card was purchased from 
AVerMedia [24] and is displayed in Figure 4.18. The capture card has a USB 2.0 interface between the laptop and the camera and provides support for MPEG 1/2/4 formats.

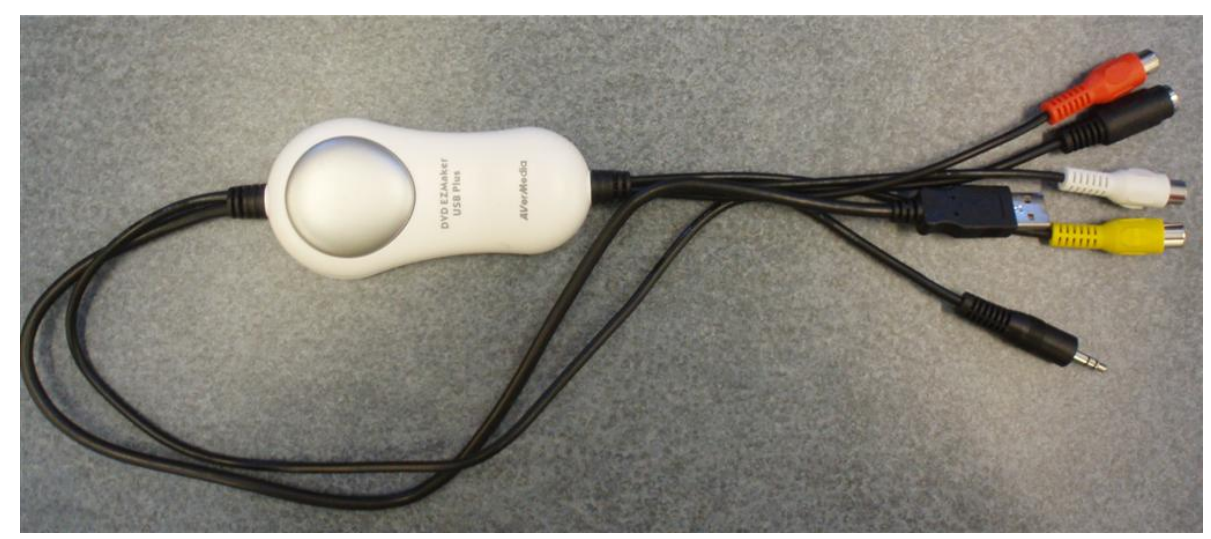

Figure 4.18: AVerMedia USB Video Capture Card

The video signal that is transmitted back from the robot connects to the capture card on the yellow connector. This image is then converted to a USB signal and displayed to the user on the software interface. The remaining connectors can be used to capture video or audio signals in different modes, but neither of these connectors are required and thus are not used. 


\section{Chapter 5}

\section{Software}

Software was designed and tested to receive user selected information from the base station computer and perform the necessary tasks. LABVIEW is used to implement the Graphical User Interface (GUI) between the robot and the user, as the author has previous experience with this software package. A GUI prototype has been implemented and is illustrated in Figure 5.1. Each component is discussed in sections that follow.

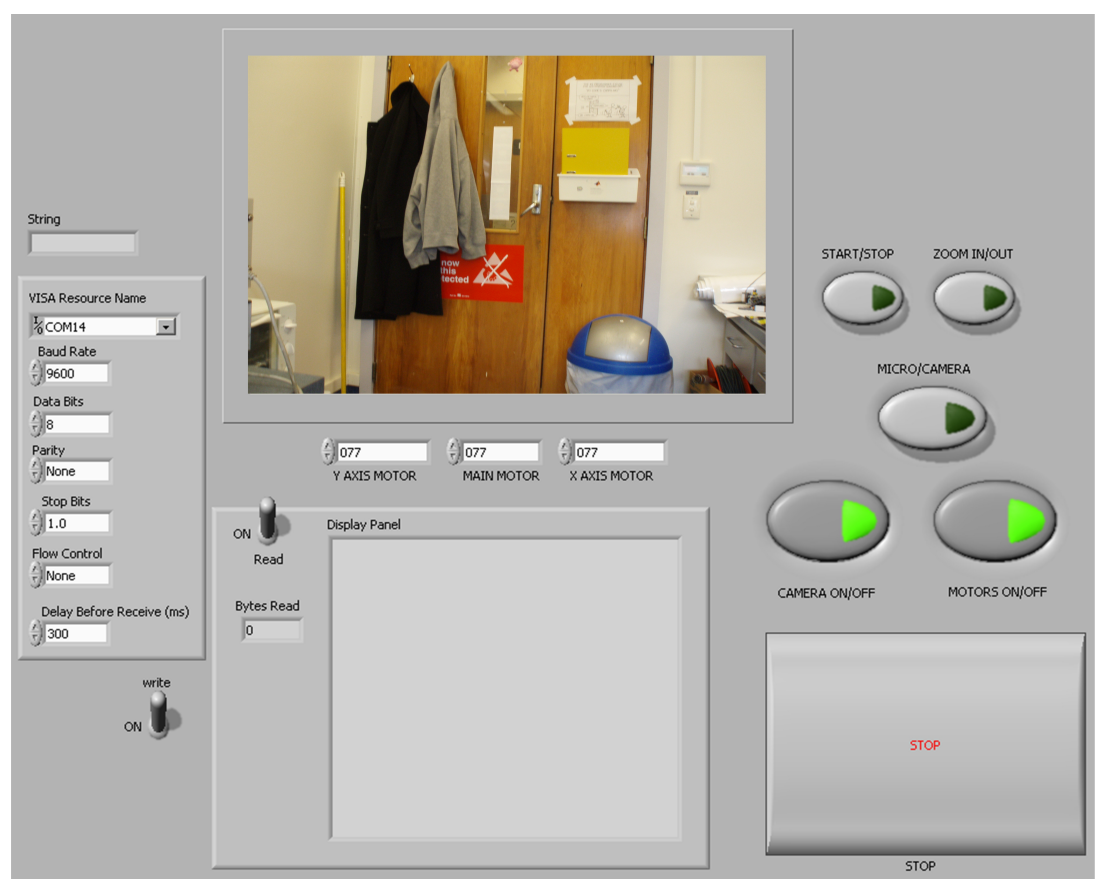

Figure 5.1: LabVIEW GUI Design 
The block diagram shown in Figure 5.2 describes the software code used for implementation and execution of the GUI interface. The diagram is never seen or modified by the user but it contains the necessary applications to enable the GUI interface to achieve expected results.

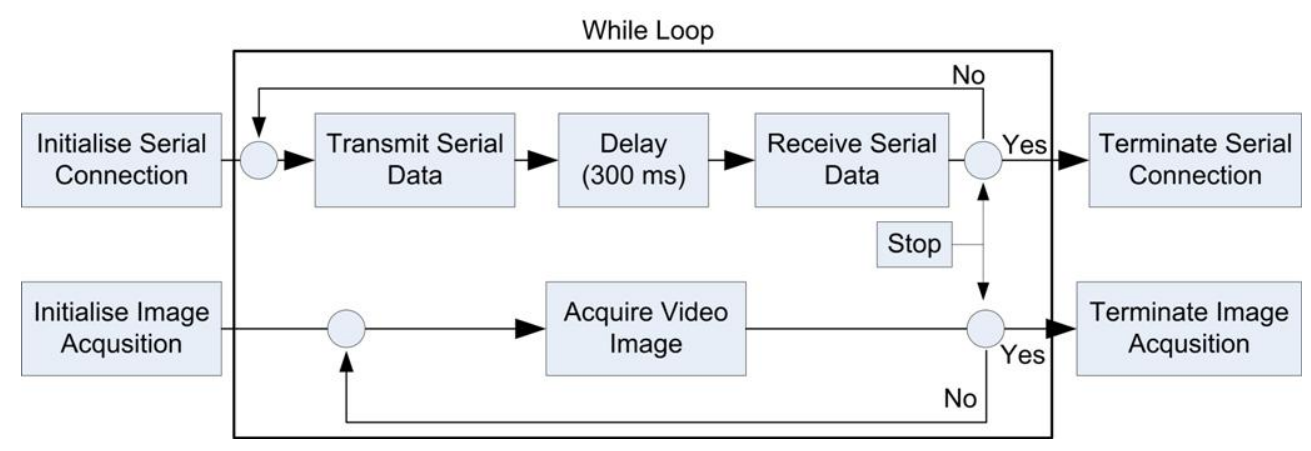

Figure 5.2: LabVIEW GUI Block Diagram

LABVIEW communicates with the micro-controller and the camera using a serial connection. The micro-controller is configured with automatic address recognition (AAR) as discussed in Section 5.1.3. The AAR enables LABVIEW to communicate with multiple devices using two data lines. This is a major improvement to the previous design which required four lines (Section 3.2.1). The AAR is a great advantage because it minimises the necessary number of communication lines between the computer, camera and the micro-controller. The complete micro-controller program is shown in Appendix B.1-B.10.

\subsection{Serial Communication}

To establish communication between the computer and the robot, a Virtual Instrument Software Architecture (VISA) application is used which is written and implemented by National Instruments. Communication is established by initialising the VISA application with the correct USB port, setting the baud rate to 9600,8 data bits, no parity and 1 stop bit. If at any stage serial communications can not be established an error message will inform the user about the problem. The initialisation code shown in 
the block diagram is executed once to establish a connection as shown in Figure 5.3 .

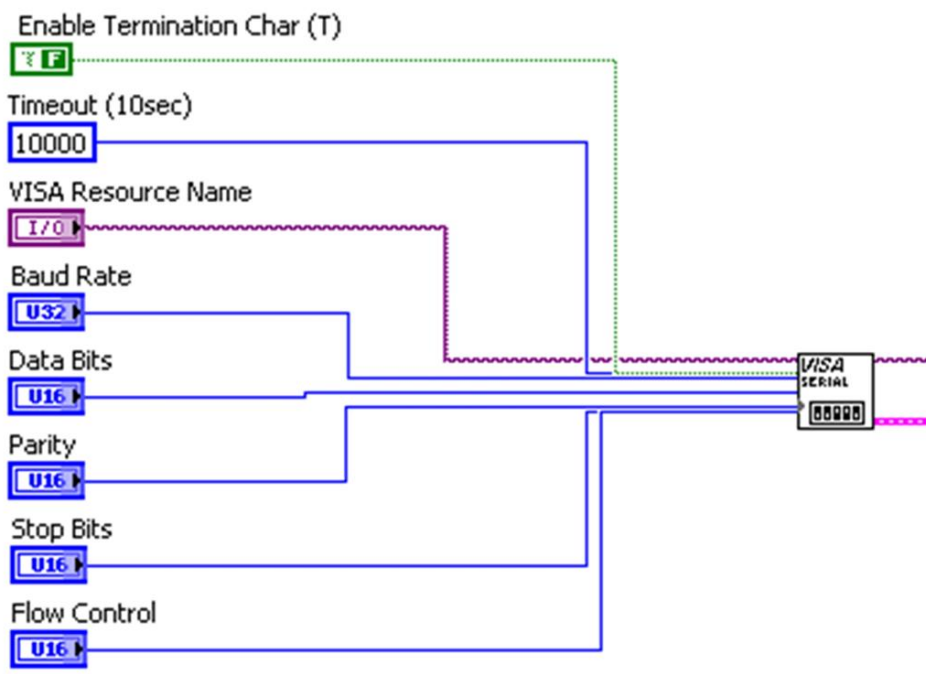

Figure 5.3: VISA Block Diagram Initialisation

The micro-controller receives the data packet and extracts the information one bit at a time. The information received is analysed and the necessary code and subroutines are executed. The code executed is stored inside the micro-controller's storage area known as a register. A register implemented inside the AT89C51AC3 micro-controller is 8-bits, bit 0 being the least significant bit (LSB).

Each bit located inside the register can be set high or low depending on the functionality of the register and purpose. In the next few sections these registers are discussed in detail to achieve desired outputs.

\subsubsection{LabVIEW Serial Transmit}

When the serial connection has been established, a while loop is repeatedly executed, transmitting data packets to the robot. A data packet transmitted from the computer to the micro-controller contains information about the selected communication device (address), target speed for all three motors and to either turn on/off the camera and the motor drivers as shown in Figure 5.4 . 


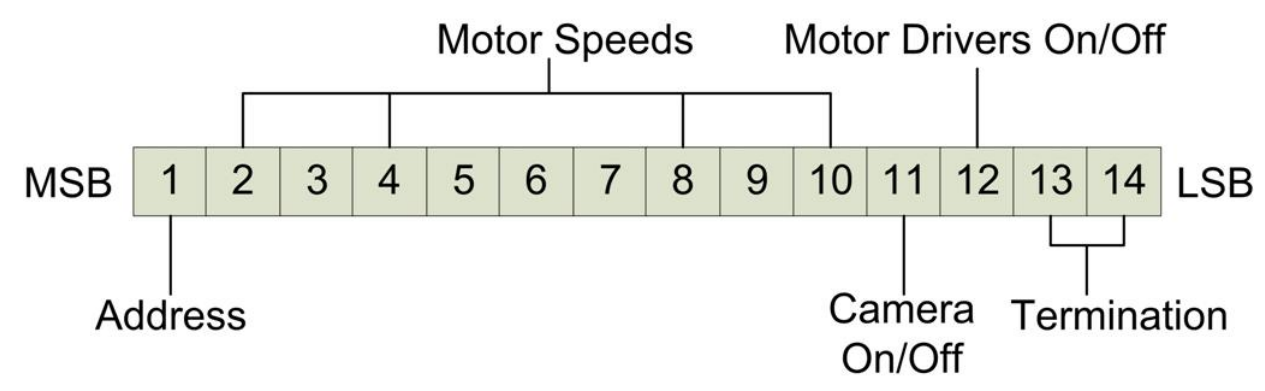

Figure 5.4: Data Packet Structure

Before the data packet is transmitted, the required information must be gathered and stored inside a string array. This is necessary because some data bits are set as integers and doubles by default but have to be converted into a string to allow the serial buffer to transmit them to the robot. The first byte to be stored inside an array is the address byte. Figure 5.5 shows motor speed values for all three motors which are stored inside the array after the address byte.

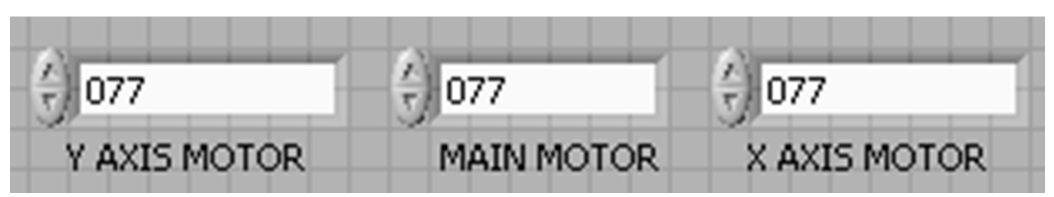

Figure 5.5: Motor Speed Values

The main motor value is stored first which is then followed by the $X$ and $\mathrm{Y}$ motor values. The micro-controller has been configured to receive three bytes for each motor value, meaning all motor values have to be padded with zeros if the value exceeds 100 . The default value for all three motors is set to 077 as discussed in Section 5.3.2.

The MICRO/CAMERA button selects the device the user wishes to communicate with. If the button is set to true, the array address byte is set to 0x61 and therefore the user has the ability to control the microcontroller, i.e. the forward and backward movement of all three motors. If the button is set to false, the array address byte is set to $0 \times 81$ and therefore the user controls the camera, i.e. the zoom in and out motors. If the camera has been selected by the user, the data packet values stored inside the 
array are pre-determined by the manufacturer as shown in Table 5.1 .

Table 5.1: Camera Commands and Packets

\begin{tabular}{|l|c|c|}
\hline Command Set & Command & VISA Packet \\
\hline \multirow{2}{*}{ Camera Power } & On & $8101040002 \mathrm{FF}$ \\
\cline { 2 - 3 } & Off & $8101040003 \mathrm{FF}$ \\
\hline \multirow{3}{*}{ Camera Zoom } & Stop & $8101040700 \mathrm{FF}$ \\
\cline { 2 - 3 } & Tele & $8101040702 \mathrm{FF}$ \\
\cline { 2 - 3 } Camera Date Display & Wide & $8101040703 \mathrm{FF}$ \\
\cline { 2 - 3 } & On & $8101072 \mathrm{a} 02 \mathrm{FF}$ \\
\hline Camera Time Display & Off & $8101072 \mathrm{a} 03 \mathrm{FF}$ \\
\cline { 2 - 3 } & On & $8101072 \mathrm{~b} 02 \mathrm{FF}$ \\
\hline
\end{tabular}

If the user wanted to execute any of the commands available, the corresponding VISA packet would have to be transmitted to the camera. The user selects which data packet to transmit by setting the START/STOP and ZOOM IN/OUT buttons to a high or a low state. If the ZOOM IN/OUT button is set to high, the camera will zoom out (Wide), but if the button is set to low, the camera will zoom in (Tele). Both commands are executed when the START/STOP button is set to true, otherwise the transmitted data would inform the camera to disengage all zoom motors. Figure 5.6 shows the ZOOM IN/OUT, START/STOP, MICRO/CAMERA, CAMERA ON/OFF and MOTORS ON/OFF buttons.

The last two bytes to be stored inside the array are the termination characters which have a value of $0 \times 66$. The reason for setting this value is because $0 \times 66$ will never be transmitted inside the data packet but if problems are encountered the value can easily be changed. The termination characters are used to inform the micro-controller that the received data packet has reached the last byte. When the information has been gathered and stored inside the array it is then placed inside the LabVIEW transmit buffer. The buffer then transmits the whole array one byte at a time to the micro-controller. Figure 5.7 shows the block diagram that is used to transmit the data array to the micro-controller. 


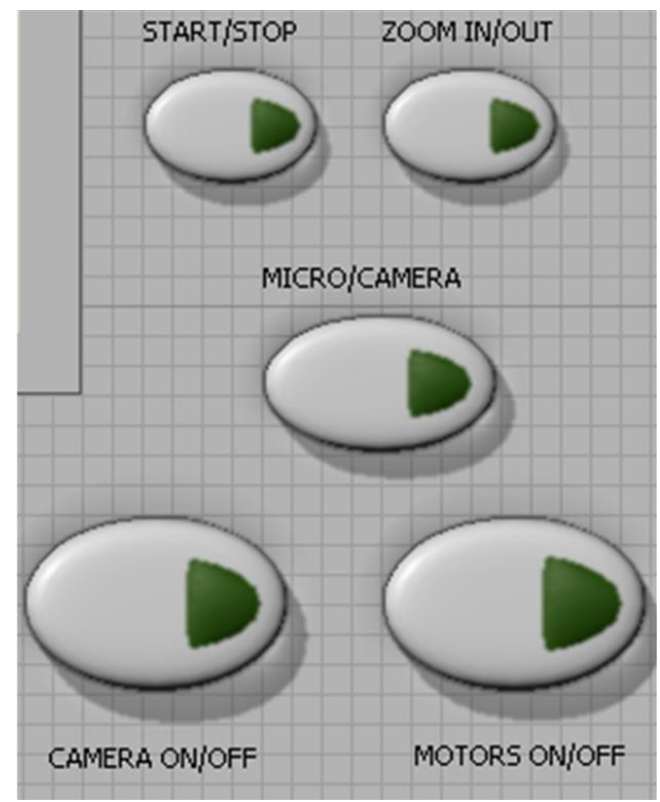

Figure 5.6: Camera \& Micro-controller Button Panel

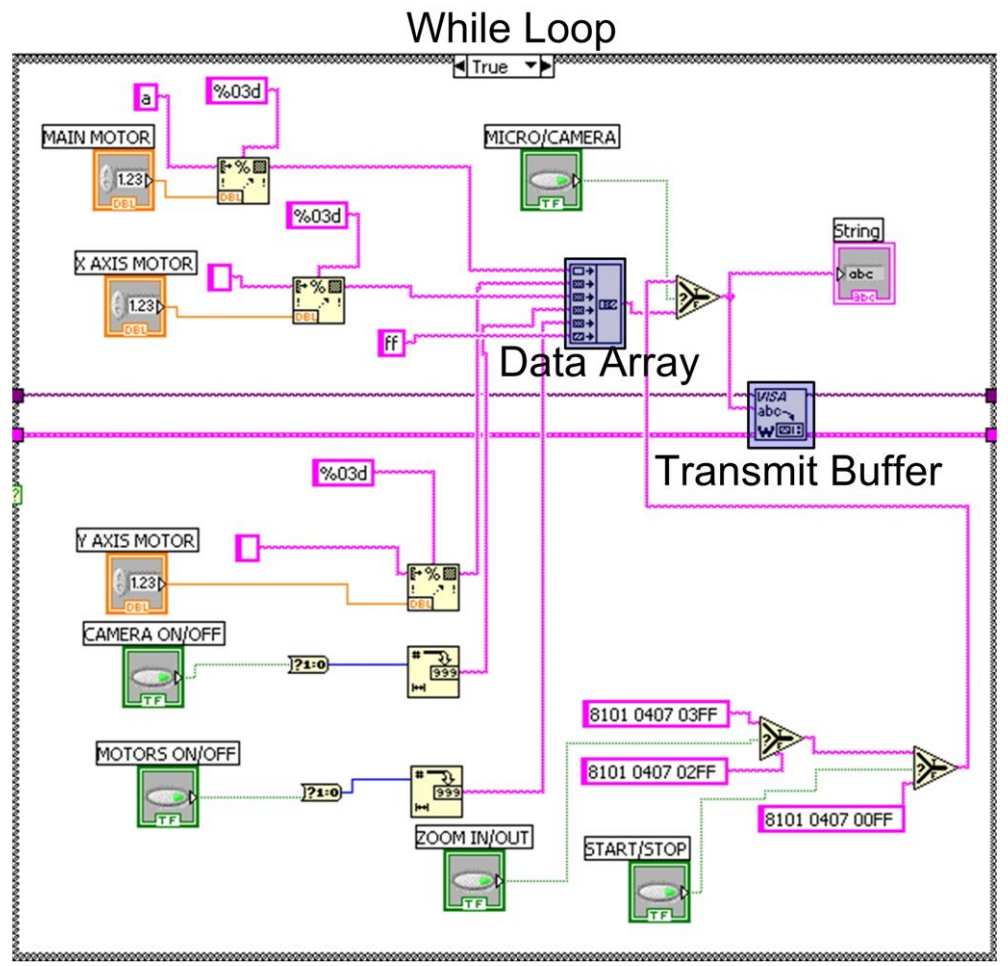

Figure 5.7: Transmit Data Block Diagram 


\subsubsection{Micro-controller Serial Initialisation}

Before the micro-controller can receive the transmitted data the serial registers have to be initialised. The micro-controller uses the RX line located on pin 0 port 3 to receive the transmitted data packet. To establish serial communication with the base station the SCON register is utilised as shown in Figure 5.8 .

\begin{tabular}{c|c|c|c|c|c|c|c|c|}
\multicolumn{1}{c}{} & 0 & 1 & 2 & 3 & 4 & 5 & 6 & 7 \\
\cline { 2 - 8 } LSB & RI & TI & RB8 & TB8 & REN & SM2 & SM1 & SM0/FE \\
\cline { 2 - 7 }
\end{tabular}

Figure 5.8: SCON Register

The SCON register configures the micro-controller in 8-bit serial transmission mode by setting the SM1 and SMO /FE bits to 1 and 0 respectively. The SCON register is configured in 8-bit mode because the LabVIEW software package is also configured in 8-bit. The SM2 is set high enabling AAR which is discussed in detail in Section 5.1.3. The REN bit is set high which enables the serial communication. The receive (RI) and transmit (TI) interrupt flags are set by hardware to notify the micro-controller when the 8th bit has been received or transmitted respectively and therefore they are initialised to 0 . All other bits inside the SCON register are set to 0 hence a value of $0 \times 07$ is assigned to the register.

To establish serial communication the SCON register requires an internal clock source to generate the baudrate. This is achieved by utilising the timer/counter mode control (TMOD) register shown in Figure 5.9.

\begin{tabular}{|c|c|c|c|c|c|c|c|c|}
\hline & 0 & 1 & 2 & 3 & 4 & 5 & 6 & 7 \\
\hline SB & M00 & M10 & C/TO\# & GATEO & M01 & M11 & C/T1\# & GATE1 \\
\hline
\end{tabular}

Figure 5.9: TMOD Register

The TMOD register can be used to configure Timer 0 (M00, M10, C/T0\#, GATE0) or Timer 1 (M01, M11, C/T1\#, GATE1) as the internal clock source. There are no advantages or disadvantages between the two timers and therefore Timer 1 was selected arbitrarily to be used for serial communication and Timer 0 was selected for the PCA modules which are discussed in 
Section 5.3.1. Timer 0 and 1 are configured in an 8-bit auto-reload mode by setting the MO1 and MOO bits to 0 and M11 and M10 bits to 1 . The reason for choosing the 8-bit auto-reload timer over a 16-bit timer is because with the auto-reload mode the timer reset is done automatically when a value of 255 is reached. The reset for the 16-bit timer would have to be handled by software and therefore unnecessary software implementation is removed, but at a cost of loosing a high accuracy clock. The remaining bits inside the TMOD register are not used and therefore a value of $0 \times 22$ is assigned to the register.

The user selected baud rate between the micro-controller and the computer is determined by the TH1 register. The baud can be calculated using Equation 5.1.

$$
\text { Baud }=\frac{F_{\text {osc }}}{192 \times(256-T H 1)}
$$

The oscillator frequency $\left(F_{\text {osc }}\right)$ is $20 \mathrm{MHz}$ and the baud rate selected is 9600. This baud rate was chosen because the camera uses the same rate and it can not be changed. Rearranging Equation 5.1 the TH1 value is calculated to be 245.149 as shown in Equation 5.2 .

$$
\begin{aligned}
& T H 1=256-\frac{20000000}{9600 \times 192} \\
& T H 1=245.149
\end{aligned}
$$

The TH1 value must be rounded because it is an 8-bit integer. If the value is rounded to 246 the baud rate is calculated to be 10416.67 using Equation 5.2 and if it is rounded to 245 the baud rate is calculated to be 9469.697 Using the value of 246 the error is calculated to be $8.5 \%$ and with the value of 245 the error is $1.36 \%$. The value of 245 provides a smaller error and therefore a value of $0 x F 5$ is assigned to the TH1 register.

The power configuration ( $\mathrm{PCON}$ ) register shown in Figure 5.10 is used to power down the micro-controller if the user wanted to save power. The SMOD 1 and SMODO bits are used to select the serial mode that the microcontroller is set in and because the mode selected is 8-bit serial the values assigned are 1 and 0 respectively. The current project does not contain a power source problem and therefore the PCON register is given a value of 0x80. 


\begin{tabular}{|c|c|c|c|c|c|c|c|c|}
\hline & 0 & 1 & 2 & 3 & 4 & 5 & 6 & 7 \\
\hline LSB & IDL & PD & GF0 & GF1 & POF & - & SMODO & SMOD1 \\
\hline
\end{tabular}

Figure 5.10: PCON Register

The values that have been assigned to the SCON, TMOD, TH1 and PCON registers are shown in Figure 5.11 .

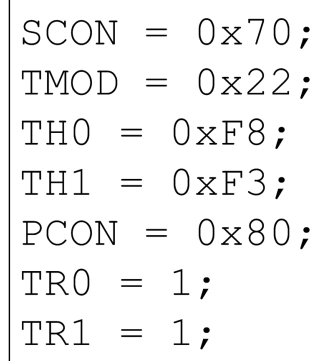

Figure 5.11: Serial Connection Initialisation

The TR 1 and TR 0 bits are used to turn timers 1 and 0 on respectively. The THO register and the value assigned to it is discussed in Section 5.3.1.

\subsubsection{Micro-controller Automatic Address Recognition}

The cable used between the robot and the base station has 10 wires. This is a problem because the number of transmission lines required exceeds the number of lines available as discussed in Section 3.2.1. To solve this problem, the micro-controller has been configured to perform AAR inside the SCON register which results in only two communication lines being required. This means the micro-controller and the camera serial ports do not examine a data packet unless its own address is recognised. The camera used inside the robot has automatic address recognition already established by the manufacturer and the default address is set to $0 \times 81$.

Inside the SCON register the SM2 is set to high when AAR is on and set to low when it is turned off. Looking at Figure 5.12 the micro-controller address is set inside the SADDR register, meaning each time a data packet is transmitted by LabVIEW the address byte transmitted must match the value stored inside the SADDR register which is arbitrarily set to $0 \times 61$. 
$\mathrm{SADDR}=0 \times 61 ;$

SADEN $=0 \times F F$;

Figure 5.12: Automatic Address Recognition

If any other byte is transmitted by LabVIEW, the micro-controller will ignore the data packet following it. The mask address inside the SADEN register provides the flexibility to address one or more devices simultaneously, but since this feature is never used a 0xFF value is assigned to SADEN.

\subsubsection{Micro-controller Serial Receive}

When a byte is received on port 3 the micro-controller uses the 8-bit serial buffer (SBUF) register to store individual bits. The same register is used to transmit individual bytes back to LabVIEW. The micro-controller has the ability to flag receive (RI) interrupt each time a byte is received and a transmit (TI) interrupt when the micro-controller has finished transmitting an individual byte. This is advantageous because the micro-controller can calculate and process data and does not need to constantly poll the receive or transmit lines.

The first byte received and stored inside the SBUF register is the SADDR address assigned to the micro-controller. The next 9 bytes received are the user values for setting the speed of all three motors which is followed by two bytes informing the micro-controller if the user wishes to engage or disengage power to the camera and motor drivers. The last two bytes received are termination bytes and are used to inform the micro-controller that the end of the data packet has been reached.

When either RI or TI flags have been set high the micro-controller must know the name of the function where the serial interrupt routine is executed. Looking at Figure 5.13 the ROM_VECTOR is used to assign the function name (Serial ()) to the serial interrupt address (0x23), meaning each time a receive or transmit interrupts are triggered the Serial () function is executed.

When the interrupt routine has been triggered, the TI flag is checked 


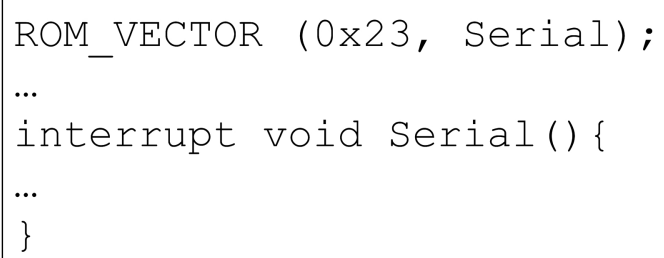

Figure 5.13: Serial Communication Initilisation

if it has been set high as shown in Figure 5.14. The reason for checking the TI flag first is because the routine executed is shorter in length compared to the RI routine and therefore the time spent inside the routine will be shorter as well. In this case the routine is short because the TI flag is reset to 0 .

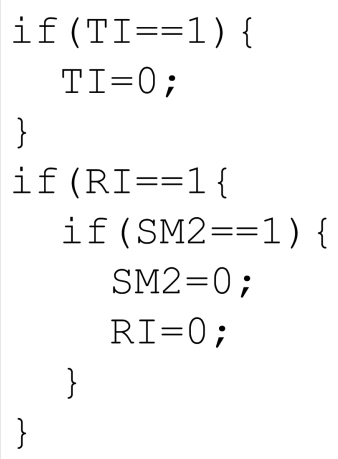

Figure 5.14: Serial Interrupt Routine Part 1

The RI flag is the second flag to be checked. If the RI flag is set high this means the first byte received inside the SBUF register matches the micro-controller's address and therefore the SM2 and RI bits can be reset to 0 . This is done because the SM2 bit turns off the automatic address recognition and therefore the remaining bytes inside the data packet can be received. The RI bit notifies the micro-controller that the information received inside the SBUF register has been acknowledged and therefore the interrupt routine can exit.

The next 9 data bytes received contain motor speed information. The first byte received is stored inside the buffer array (buffer [i]) global variable as shown in Figure 5.15 .

The buffer array is used to store the bytes received and then later anal- 


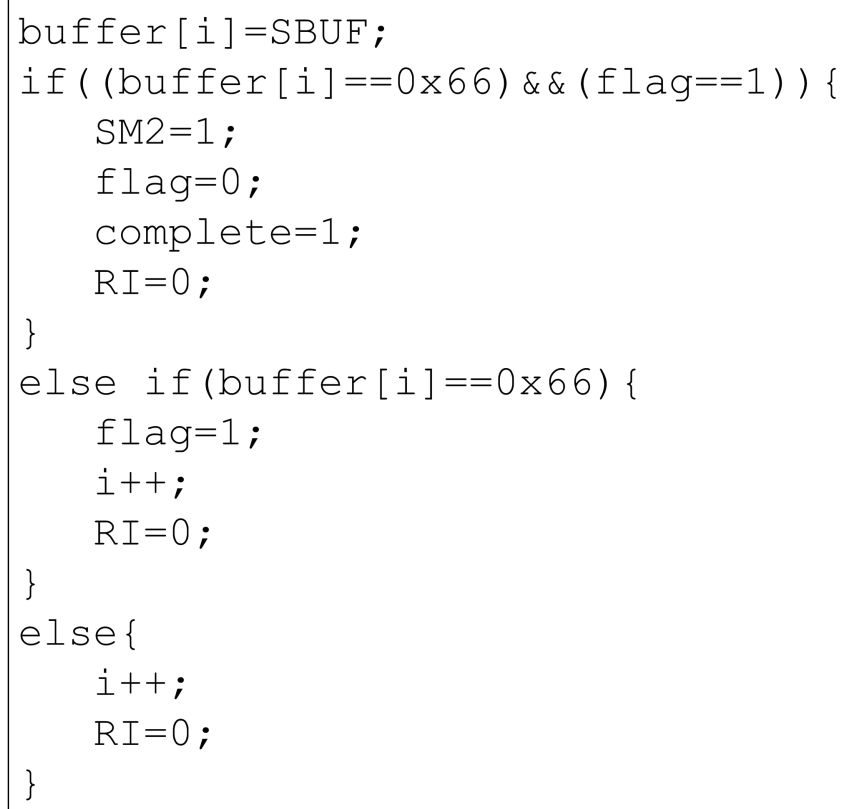

Figure 5.15: Serial Interrupt Routine Part 2

ysed when termination values have been reached. The $i$ value located inside the buffer is used as a global counter which is incremented each time a new byte is received. The counter increment is necessary to avoid old bytes from being overwritten with the new ones. Each time a byte is stored inside the array it is checked against the termination byte value of $0 x 66$. If the values match, a global receive flag ( $\mathrm{flag}$ ) is set to high notifying the micro-controller that the first termination byte has been received and the next byte received should also be a termination byte.

When both termination characters have been received the SM2 bit is reset back to high turning the AAR back on. The flag is reset to 0 and the global complete flag (complete) is set to high notifying the microcontroller that the whole data packet has been received and it can be analysed outside the interrupt routine.

\subsubsection{Micro-controller Serial Transmit}

To eliminate any software problems that might occur, the micro-controller is configured to transmit data back to the LabVIEW panel display. The micro-controller uses the TX line located on pin 1 port 3 to transmit data 
back. These data contain information about the current status of the motor drivers and the camera, but this can be changed if the user needs more or different information to be displayed. Before data are transmitted the information is gathered and placed inside the character array.

Problems were encountered when the micro-controller would try and communicate with the computer via the RS-485 transceiver, similar to the problem discussed in Section 4.4.2. In the previous section the problem was solved using a hardware configuration but the main board does not contain a FT232BQ chip and therefore a software approach had to be employed.

The main board was designed to enable the micro-controller to engage and disengage the RS-485 transmit and receive drivers independently. As discussed in Section 4.2.3 the drivers are controlled by setting the RXEN and DXEN pins on the RS-485 transceiver. The micro-controller pins 0 and 1 on port 0 are directly connected to the RXEN and DXEN pins respectively as shown in Figure 5.16 .

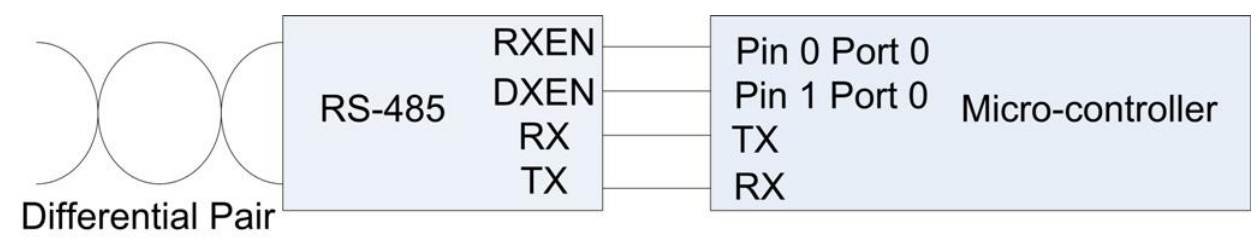

Figure 5.16: RS-485 Configuration

Inside the micro-controller pin 0 on port 0 is assigned a variable name RXEN and pin 1 is assigned DXEN. Initially the RXEN and DXEN variables are set to 1 and 0 respectively. This is done because the micro-controller will receive data first from LabVIEW before transmitting information back. To eliminate the data reflection problem discussed in Section 4.4.2 the RXEN bit is set to 0 (turning the receive driver off) which is then followed by the DXEN bit being set to 1 (turning the transmit driver on) as shown in Figure 5.17

This sequence is necessary because the RXEN pin on the RS-485 chip has to be shut down first before the DXEN pin is enabled, resulting in no transmitted data being returned on the received line. The character 
$\mathrm{RXEN}=0$;

$\mathrm{DXEN}=1$;

WriteLn (output);

$\mathrm{DXEN}=0$;

$\mathrm{RXEN}=1$;

Figure 5.17: RS-485 Transmitting Data

array (output) containing the information is then transmitted using the WriteLn function and the DXEN and RXEN variables are reset back to their default values.

Figure 5.18 shows the WriteLn ( ) function receiving an array of characters to be transmitted. The array is then broken into individual characters to be transmitted to LabVIEW.

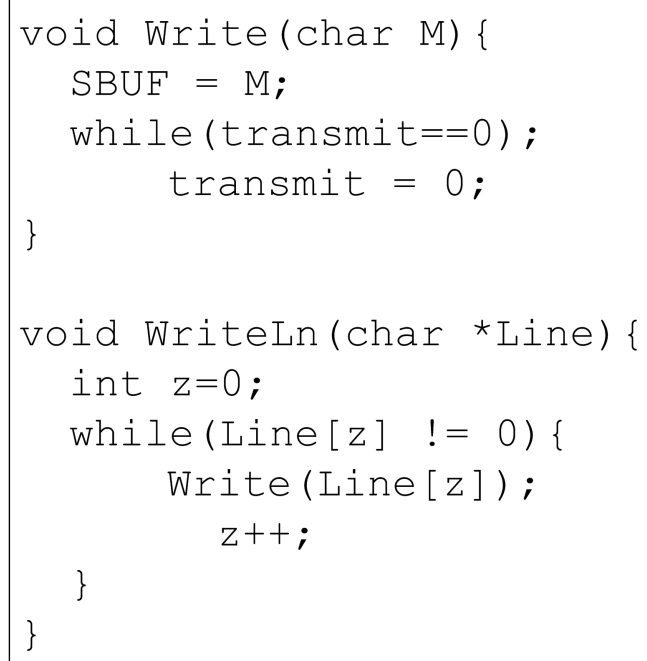

Figure 5.18: Data Transmit Routine

The Writeln () function contains a while loop which checks individual characters inside the array for a null value. If a 0 character has not been reached the WriteLn() calls the Write() function to transmit a character to the computer using the SBUF register. The z counter index is incremented to retrieve the next character inside the array. If a null character has been reached the while loop and the Writeln () function are terminated. 


\subsubsection{LabVIEW Serial Receive}

As seen in Figure 5.2 there is a 300 ms time delay between transmitting and receiving the serial data. This time delay is necessary because it allows LabVIEW to finish transmitting the whole data packet to the robot before the receive routine is executed. If there is no delay between the routines the data packet stored inside the transmit buffer would be overwritten by the received data packet. When the time delay has expired the transmitted data from the micro-controller are shown to the user on the display panel as shown in Figure 5.19 .

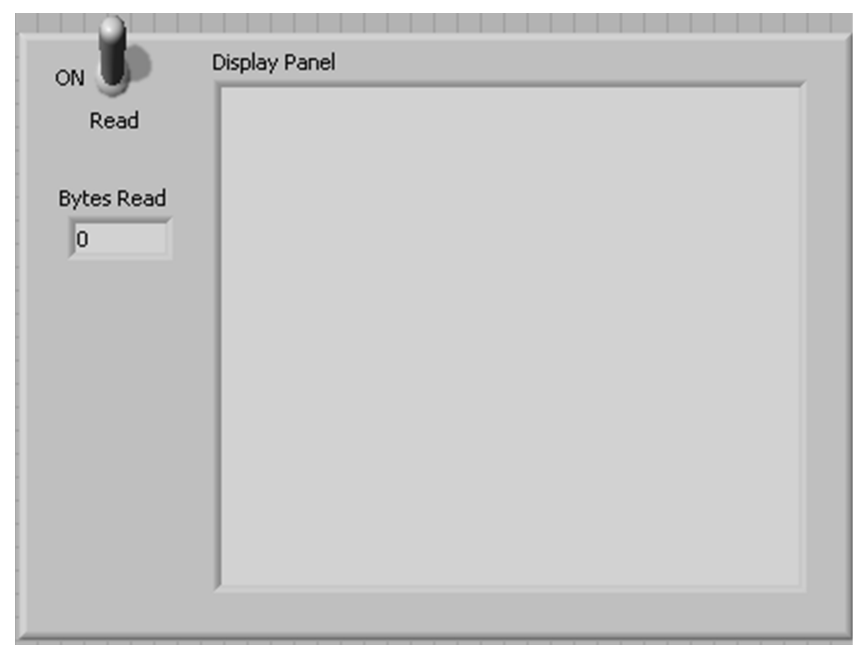

Figure 5.19: LabVIEW Display Panel

The display panel also contains a read (Read) switch which is used to turn the receive buffer on or off. This switch is used to isolate a serial communication problem that might occur by turning the receive switch off. The user also has the information about the number of bytes that are received. This feature is useful if the number of bytes being received is the same on every transmission, but because the number of bytes received changes constantly this feature is not utilised. Initially the data received are stored inside a VISA buffer which is then shown on the display panel.

When all of the data have been received from the micro-controller the serial process will restart from the start. The process executes until the stop (STOP) button has been pressed. 
When the stop button has been pressed the LabVIEW software terminates the serial connection that was established and allows other devices to use the USB port. The block diagram used and implemented for the time delay, receive buffer and the serial connection termination is shown in Figure 5.20

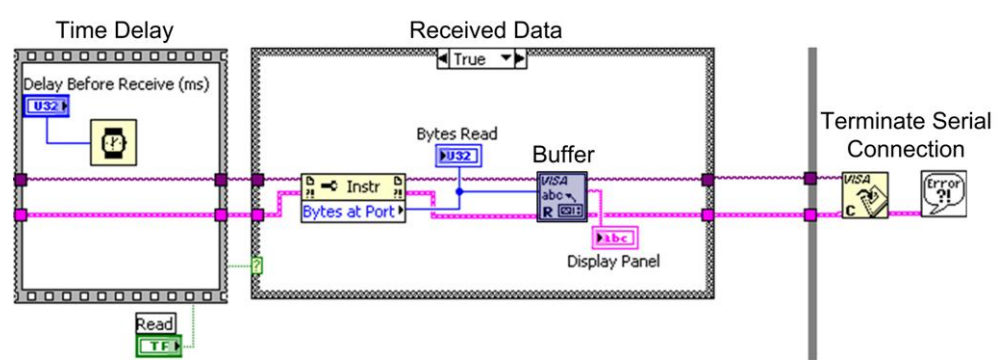

Figure 5.20: Receive Data Block Diagram

\subsection{LabVIEW Image Acquisition}

Figure 5.2 shows how the image acquisition is initialised from the robot's camera in parallel with the serial connection. The initialisation is done separately using a image acquisition application which is written by National Instruments. For the image to be detected by the computer, a video capture card is used as discussed in Section 4.4.3. The video capture card is connected to the USB port which connects to the output of the video camera. The video image is displayed until the user presses the stop button. When the button has been pressed the image acquisition connection is terminated and LabVIEW releases the USB connection for other devices to use as shown in Figure 5.21 .

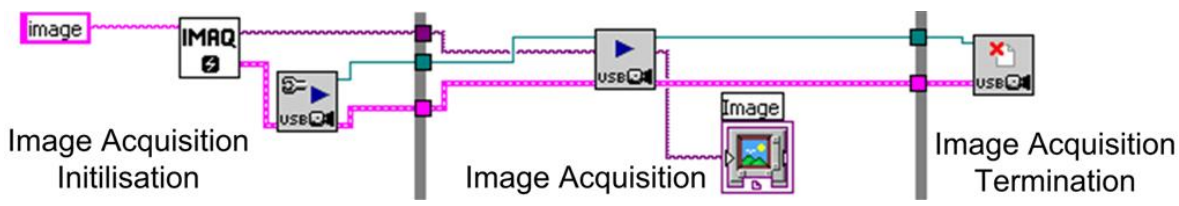

Figure 5.21: Block Diagram Video Acquistion 
The while loop is repeatedly executed which results in a constant video image stream being displayed to the user on the video panel as shown in Figure 5.1 .

\subsection{Micro-controller Motor Control}

The motor speed information contained inside the received data packet must be analysed and the appropriate registers must be utilised to achieve a correct PWM signal. As indicated in Figure 4.6 the motor drivers require a PWM signal input on the S1 and S2 terminals in order to output a voltage difference across motor terminals M1 and M2. The micro-controller has five PCA modules and each module can be configured either as a software timer, high speed output, rising and/or falling edge capture or a pulse width modulator.

\subsubsection{PCA Initialisation}

To configure the PCA module as a pulse width modulator the CCAPM register as shown in Figure 5.22 is utilised.

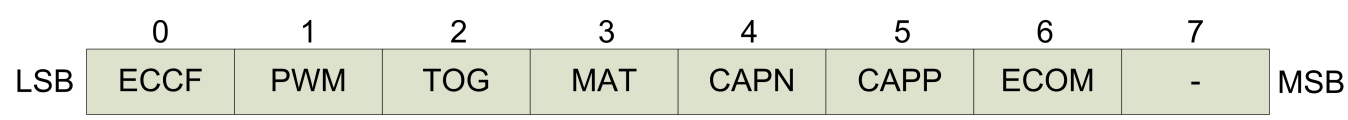

Figure 5.22: CCAPM Register

The pulse width ( $\mathrm{PWM})$ and enable compare (ECOM) bits must be set high to enable the PWM module. The CAPP, CAPN, MAT, TOG and ECCF bits are set low because they are not currently used and therefore a value of $0 \times 42$ is assigned to the CCAPM register. The output for PCA module 2 is located on pin 6 of port 1 and it controls the main motor. PCA modules 3 and 4 are located on pins 7 and 8 of port 1 and are used to control the $X$ and $Y$ motor axes respectively. The Interrupt Enable Register (IENO) shown in Figure 5.23 is used to turn on the serial (ES) and PCA (EC) interrupts.

The EA bit must be set to high to enable all interrupts, meaning each interrupt can now be individually enabled or disabled. The remaining bits 


\begin{tabular}{|c|c|c|c|c|c|c|c|c|}
\hline & 0 & 1 & 2 & 3 & 4 & 5 & 6 & 7 \\
\hline SB & EXO & ETO & EX1 & ET1 & ES & ET2 & EC & EA \\
\hline
\end{tabular}

Figure 5.23: IEN0 Register

inside the register are used to enable or disable the external (EX0, EX1) and timer (ET $, E T 1, E T 2)$ interrupts. The PWM module does not require these bits to be set high and therefore the IENO register is set to $0 \mathrm{xD} 0$.

The PCA counter mode register (CMOD) which is shown in Figure 5.24 configures the clock frequency that the PCA module will run at.

\begin{tabular}{|c|c|c|c|c|c|c|c|c|}
\hline & 0 & 1 & 2 & 3 & 4 & 5 & 6 & 7 \\
\hline SB & ECF & CPSO & CPS1 & - & - & - & WDTE & CIDL \\
\hline
\end{tabular}

Figure 5.24: CMOD Register

The PCA counter can be configured to either use a clock that runs at $\frac{F_{\text {osc }}}{2}, \frac{F_{\text {osc }}}{6}$, Timer 0 overflow or an external clock source by setting the CP S 0 and CPS 1 bits. Initially the CPSO and CPS1 bits were set to 1 and 0 respectively which selected the $\frac{F_{\text {osc }}}{2}$ clock source. The reason for choosing the $\frac{F_{\text {osc }}}{2}$ clock source is because this gives a $19.53 \mathrm{kHz}$ frequency PWM wave as shown in Equation 5.3. This clock source produces pulse widths between 0.05 and $13.10 \mathrm{~ms}$.

$$
\begin{aligned}
\text { Freq } & =\frac{F_{\text {osc }}}{4 \times 256} \\
\text { Freq } & =19.5312 \mathrm{kHz}
\end{aligned}
$$

Problems were encountered when using the $\frac{F_{\text {osc }}}{2}$ clock source because the motor drivers require a PWM pulse width between 1 and $2 \mathrm{~ms}$, i.e., a maximum frequency of $500 \mathrm{~Hz}$. This is solved by selecting Timer 0 overflow as the clock source which is configured by setting CPSO and CPS1 bits to 0 and 1 respectively and therefore assigning a value of $0 x 05$ to the $\mathrm{CMOD}$ register. The values assigned to $\mathrm{CCAPM}, \mathrm{CMOD}$ and IENO registers are 
shown in Figure 5.25 along with the PCA timer control bit (CR) which is set to 1 to enable the counter.

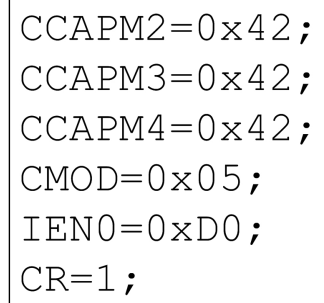

Figure 5.25: PCA Initialisation

Timer 0 can be configured to have a frequency of $500 \mathrm{~Hz}$ by setting the THO register to 242.9792 using Equation 5.4 .

$$
\begin{aligned}
& \text { Freq }=\frac{F_{\text {osc }}}{12 \times 256 \times(256-\text { TH0) }} \\
& T H 0=256-\frac{20000000}{12 \times 256 \times 500} \\
& T H 0=242.9792
\end{aligned}
$$

The THO register value must be rounded up or down because it is an 8bit integer. If the value is rounded up to 243 the frequency is calculated to be $500.8 \mathrm{~Hz}$ which exceeds the maximum frequency of $500 \mathrm{~Hz}$ and because of this the value must be rounded down to 242 , or 0xF2 in hexadecimal.

Configuring Timer 0 as an 8-bit timer in auto-reload requires setting the $\mathrm{MO} 0$ and $\mathrm{MO} 1$ bits to 0 and 1 respectively as discussed in Section 5.1.4 and therefore assigning a value of $0 \times 22$ to the TMOD register. The THO value can be seen in Figure 5.26 along with the TRO bit which is set to 1, turning timer 0 on.

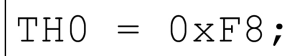

Figure 5.26: PCA Timer 0 Configuration 


\subsubsection{PWM Output \& Control}

When the data packet has been received and stored inside the character array it is then analysed inside the main while loop (while (TRUE)). Before the information can be analysed the serial (setup ()) and the PCA (PCASetup ()) initialisations have to be executed. The control transmit line (DXEN), camera power (CAMERA) and motor driver power (MOTORS) are assigned to a default value of 0 , and the receive control line (RXEN) is set to 1 as shown in Figure 5.27 .

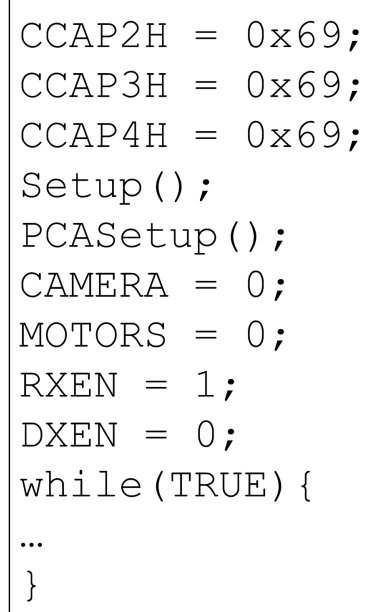

Figure 5.27: Motors, Camera, Serial and PCA Initialisation

The PCA high byte compare/capture ( $\mathrm{CCAPnH}$ ) registers are used to set the motor PWM pulse width. The CCAP $2 \mathrm{H}, \mathrm{CCAP} 3 \mathrm{H}$ and $\mathrm{CCAP} 4 \mathrm{H}$ registers are used to set the pulse widths for the main, $\mathrm{X}$ axis and $\mathrm{Y}$ axis motors respectively. The PWM pulse width must be between 1 and $2 \mathrm{~ms}$ as discussed in Section 4.2.1. To avoid exceeding the minimum and maximum pulse widths the CCAPnH values are calculated using Equation 5.5 .

$$
\begin{aligned}
& P W M_{\text {width }}=256-C C A P n H \frac{12 \times(256-T H 0)}{F_{\text {osc }}} \\
& P W M_{\text {width }}=256-C C A P n H \frac{12 \times(256-242)}{20000000}
\end{aligned}
$$

Substituting a value of 242 for the THO register the CCAPnH value is calculated to have a value of 18 for a 2 ms pulse width and a value of 137 
for a $1 \mathrm{~ms}$ pulse width. The stop mode for the motor drivers requires a pulse width of $1.5 \mathrm{~ms}$ and therefore the CCAPnH registers are calculated to be 77.43. The CCAPnH is an 8-bit register and therefore the values had to be rounded to either 77 or 78 . In the end either of the values could be chosen because it would not make a difference in the motor driver signal outputs as discussed in Section 6.1.4. A value of 77 was arbitrarily chosen.

The program enters the while(TRUE) loop when the initialisation functions have been executed. The data stored inside the array are ASCII characters and therefore they need to be converted to hexadecimal values. This is solved by creating a look up ASCII table inside the micro-controller program to convert each character value into a hexadecimal.

There are three motor speed values stored inside the array which are converted. Each value is divided into three numbers representing the hundreds, tens and ones. The CCAPnH register is calculated by multiplying each value by the appropriate constant, i.e. the hundreds are multiplied by 100 and the tens are multiplied by 10 . The multiplied constants are added together and set to the $\mathrm{CCAP} 2 \mathrm{H}, \mathrm{CCAP} 3 \mathrm{H}$ and $\mathrm{CCAP} 4 \mathrm{H}$ registers as shown in Figure 5.28. The different pulse widths that can be achieved with different values are discussed in Section 6.1.4.

$\mathrm{CCAP} 2 \mathrm{H}=((100 *$ hundreds 2$)+(10 *$ tens 2$)+$ ones 2$) ;$

$\mathrm{CCAP} 3 \mathrm{H}=((100 *$ hundreds 3$)+(10 *$ tens 3$)+$ ones 3$) ;$

$\operatorname{CCAP} 4 \mathrm{H}=((100 *$ hundreds 4$)+(10 *$ tens 4$)+$ ones 4$)$;

Figure 5.28: Motor Speed Calculation \& Output

The motor driver circuit protection that is designed inside the circuit board and discussed in Section 4.2.1 is used to send a feedback to the micro-controller about the current state of the main motor (MOT1) and X \& $\mathrm{Y}$ axes motors (МОт2) as shown in Figure 5.29.

If the user has requested for the motor drivers to be turned on, the MOT1 and MOT2 signals are checked to see if either of the bits are set low, indicating a problem. If a problem has occurred an error message (ERROR MOTORS) will be displayed to the user. In this case the user should shut down the robot and solve the problem before turning the robot back on. 


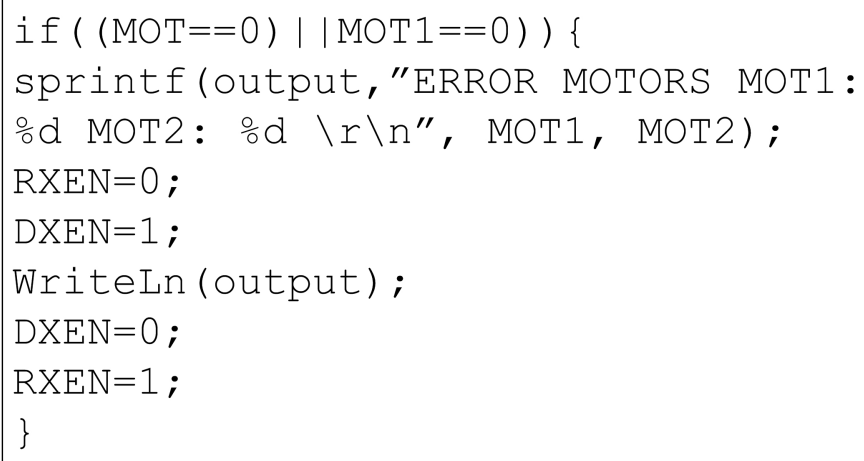

Figure 5.29: Motor Drivers Error Message

A similar circuit configuration has been implemented for the camera board as discussed in Section 4.3.2. The voltage comparator output for the camera power (CAMPOWER) is connected to pin 6 on port 2 and the voltage output for the power to the communication devices (CAMCOMM) is connected to pin 5 on port 2. The same logic is applied here as for the motor drivers. If the user turns on the camera power and communication devices a high signal should be noticed on both pins and therefore both bits would be set high, but if a low signal is received on either of the pins an error message ( $E R R O R \quad C A M E R A)$ is displayed to the user. The error code implemented is show in Figure 5.30.

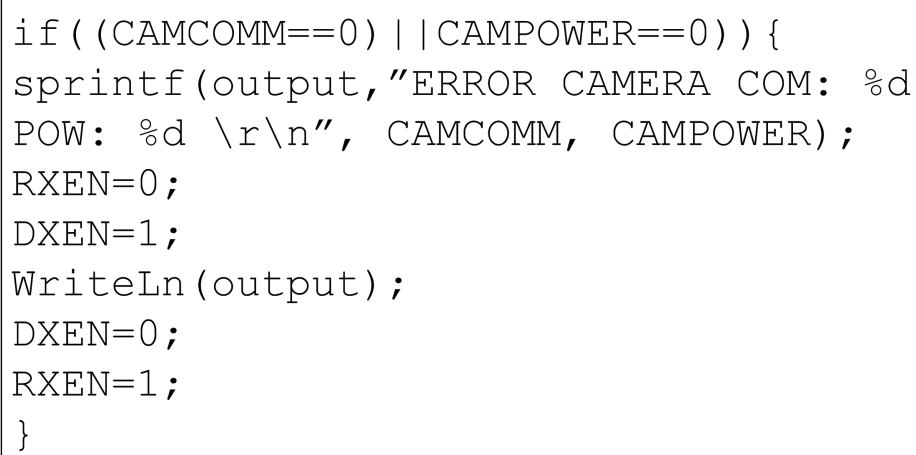

Figure 5.30: Camera Power \& Communication Error Message 


\section{Chapter 6}

\section{Results}

\subsection{Printed Circuit Boards}

The manufactured printed circuit boards (PCB) have been through three design iterations. The first redesign required a larger board in order to add larger components. The second redesign required a new and different approach towards the motor drivers and the need for additional boards because the original concept encountered design flaws. The third redesign focused on solving the communication issues that have been encountered and introduced necessary circuit protection for the main and camera boards as discussed in Sections 4.2.1 and 4.3.2.

\subsubsection{Version One}

Version one of the main board concentrated on the design and implementation of a single chip which had internal full bridge drivers and switching devices. The previous working robot had a similar configuration implemented and therefore the same approach was taken. A design consideration was to the number of electrical signals required to achieve robot control.

The first motor board constructed used the L6206D motor driver chip and the specifications are discussed in detail in Section 2.3.2. In the first circuit design no considerations were taken regarding the circuit protection due to assumptions being made that the motor operating voltages 
would not be exceeded. The first motor driver board that was designed is shown in Figure 6.1 and measured $30 \times 40 \mathrm{~mm}$.

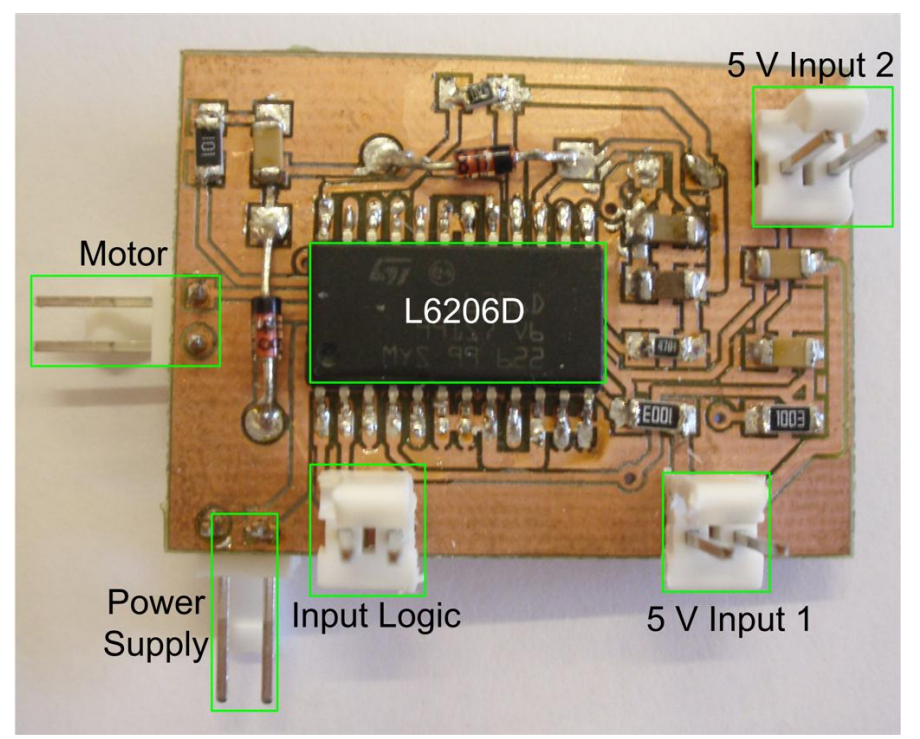

Figure 6.1: Motor Driver Board Version One

This met the room constraint inside the robot's internal enclosure. The input logic terminals are connected to pins 1 and 2 of the motor driver chip and they are used to set the rotational direction of the motor. To achieve forward or backward movement the input logic levels have to be different to each other, i.e. if pin 1 is set high pin 2 must be set low. If at any stage both pins are set at the same logic levels the motor is set in stall mode. The design intent was to set the logic inputs using a micro-controller.

Looking at Figure 6.2 the " $5 \mathrm{~V}$ Input 1" terminal is connected to pins 9 and 14 on the motor driver chip and the " $5 \mathrm{~V}$ Input 2" terminal is connected to pins 4 and 23. Each terminal is responsible for controlling an independent motor driver. If the pins are set to high the corresponding motor driver is enabled, otherwise it is disabled. The reason for designing and implementing these terminals is to provide the user with the ability to independently control each motor.

When the motor driver board was populated and turned on, a large amount of heat was being dissipated by the chip. The excess heat was removed by attaching a heat sink on top of the chip. This solution was 


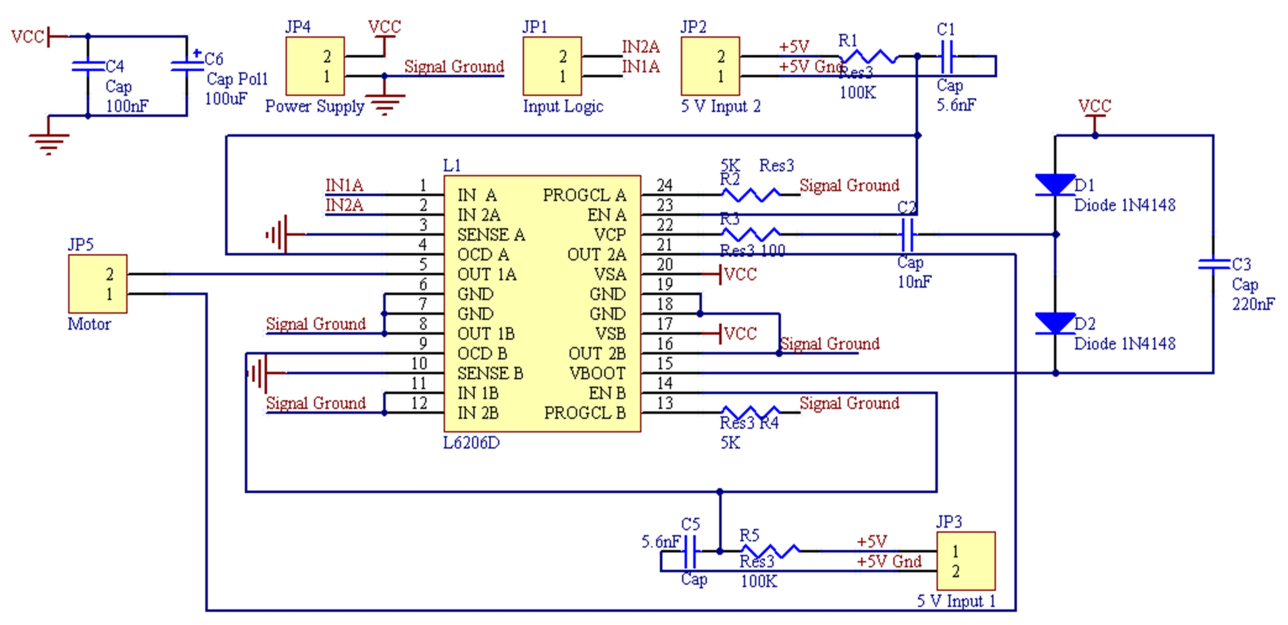

Figure 6.2: Motor Driver Board Schematic Version One

successful but was impractical because the excess heat would shorten the life expectancy of the chip and it would have to be replaced in the near future. A redesign of the main board was therefore undertaken to eliminate this problem.

\subsubsection{Version Two}

Version two of the design concentrated on implementing a motor driver circuit using independent switching devices and full bridge drivers. This was done because the full bridge drivers do not generate a large amount of heat and the switching devices are designed to handle a large current. Another advantage with the switching devices is the ability to attach them to any metal frame using a screw and a heat sinking paste to dissipate the heat generated.

The motor driver board that was designed and constructed used the HIP4081 full bridge driver chip (discussed in Section 2.2.5) in conjunction with four FGA90N30D IGBTs which are rated at $300 \mathrm{~V}$ and a continuous current of $90 \mathrm{~A}$. This design of the motor driver board also allowed for each motor to be controlled using one PWM signal therefore minimising the number of control signals that would be needed. The second revised version of the motor driver board is shown in Figure 6.3 .

The board design includes two resettable fuses to protect the switching 


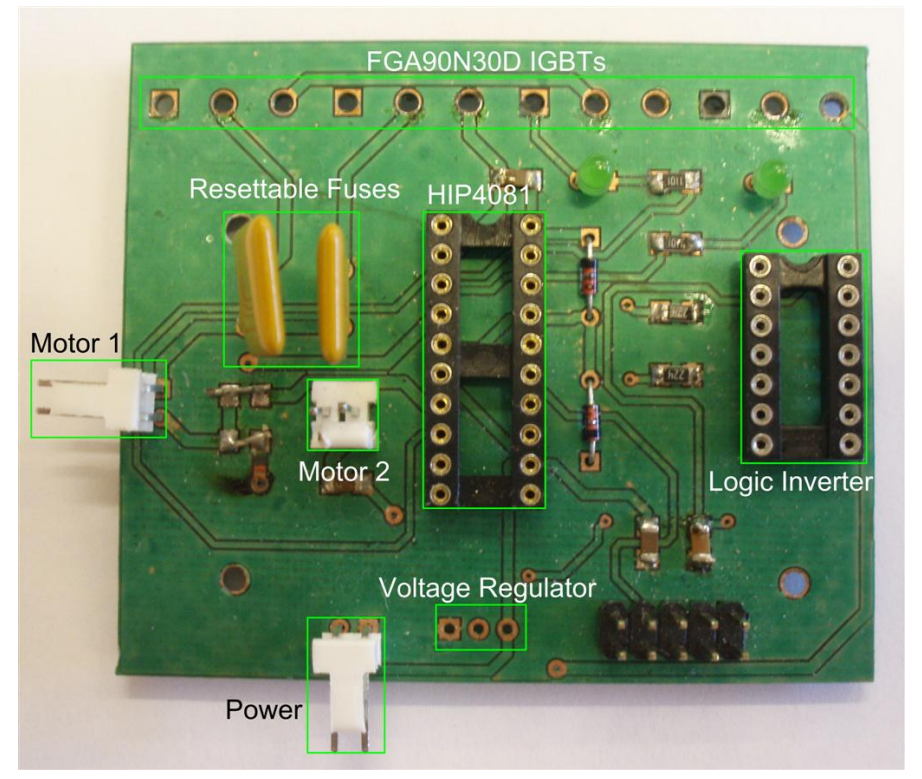

Figure 6.3: Motor Driver Board Version Two

devices from excess current being applied to the pins. The fuses used are rated at $2.5 \mathrm{~A}$, i.e. if the current drawn exceeds the limit the fuses will turn off until the power source is reset. The logic inverter located on the board is needed because the bridge driver requires an inverted input signal from the micro-controller. The rails used to deliver power to the IGBTs measure $0.6 \mathrm{~mm}$ in width instead of $0.2 \mathrm{~mm}$ due to an error in the previous design where some rails were destroyed due to high current. The schematic of the motor driver board version two can be seen in Figure 6.4.

When the board was attached to a $12 \mathrm{~V}$ DC power supply it did not function properly, with motors rotating in one direction but not the other. Subsequent to the submission of this thesis it was realised that the possible problem were the LEDs that were located between the motor driver and the switching devices. The possible solution would be to either add extra resistors after the LED and connect them directly to ground or remove the LEDs all together. 


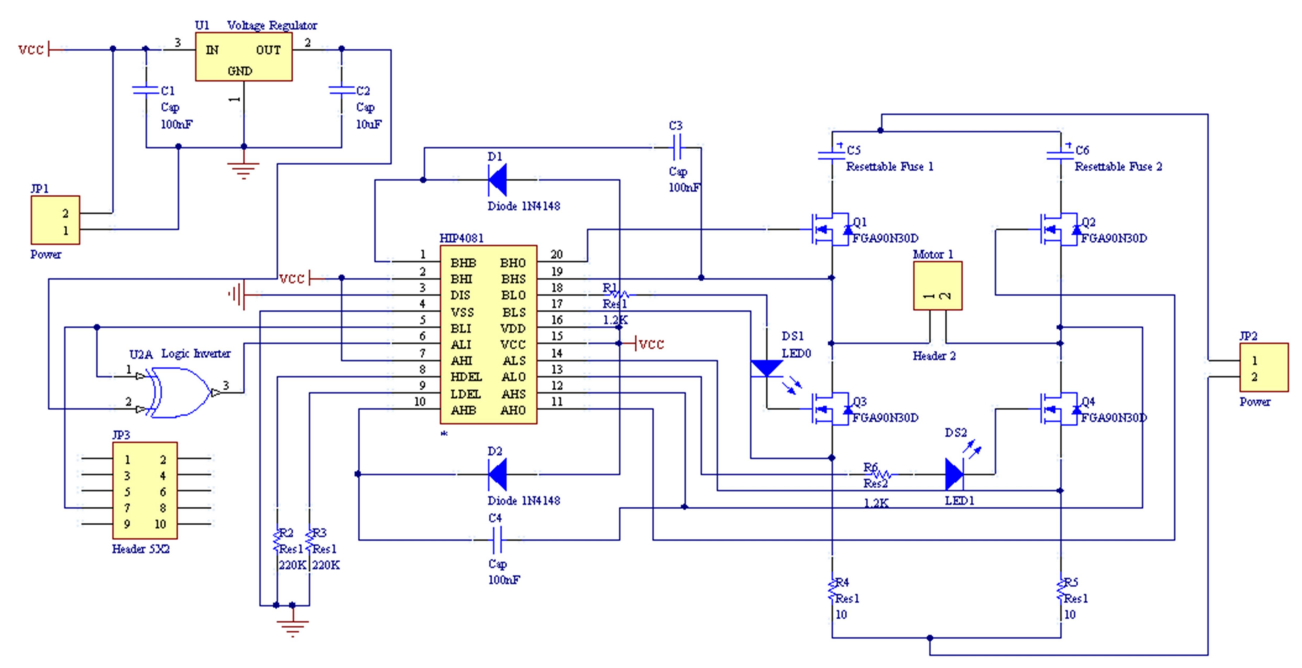

Figure 6.4: Motor Driver Board Schematic Version Two

\subsubsection{Version Three}

The SaberTooth2X5 motor driver boards were purchased and implemented as discussed in Section 4.2.1. After two redesigns of the motor driver boards it was noticed that additional boards were required to enable communication between the micro-controller and the computer, and therefore a base station board and a camera board had to be designed as discussed in Section 4.3 .2 and Section 4.4 respectively.

\section{Main Motor Board}

The initial design enabled the motor drivers to be attached directly to the main board, but this design was disregarded because the motor drivers had to be attached to a metal frame to dissipate the heat generated. The main motor board designed measures $14 \times 60 \mathrm{~mm}$. This size was chosen because the internal compartment of the robot measures $320 \times 70 \mathrm{~mm}$. The board has a micro-controller, RS-485 \& SP3283 communication devices and header pins to deliver power and control signals to the motor drivers. The third revised version of the main board is shown in Figure 6.5 .

The RS-485 and the SP3238 communication devices are located in close proximity to the micro-controller to allow the user to easily debug any communication problems that might occur. The $5 \mathrm{~V}$ regulator is located 


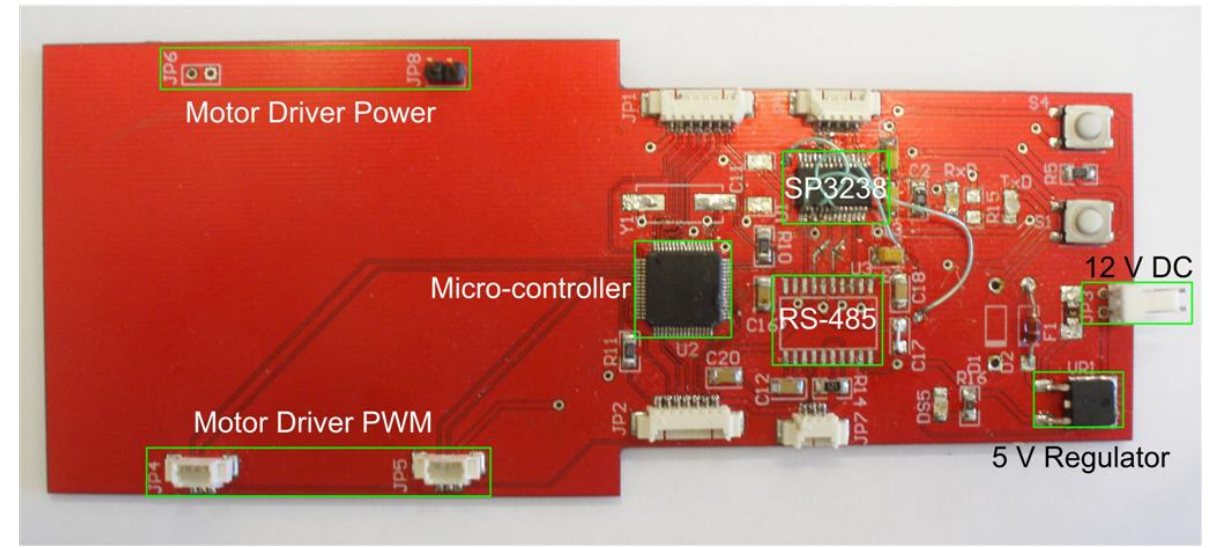

Figure 6.5: Main Driver Board Version Three

close to the $12 \mathrm{~V}$ power connector to satisfy the space constraint. The problems encountered with this design were the communication errors discussed in Section 4.4.2 and the circuit protection that had to be implemented as discussed in Section 4.2.1.

\section{Camera Board}

A board had to be designed and implemented to enable the user to have control over the camera. The camera board would be powered by the DC to $\mathrm{DC}$ converter and use the $8 \mathrm{~V}$ regulator to power the camera and the communication device as shown Figure 6.6.

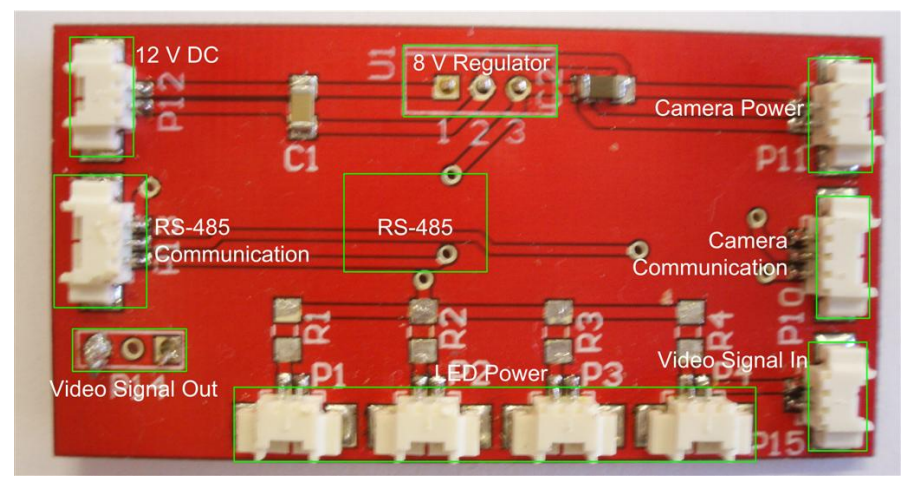

Figure 6.6: Camera Board Version Three

Initially the $8 \mathrm{~V}$ regulator was purchased in a surface mount package 
but was later changed to a through hole because too much heat was being dissipated and thus had to be attached to the internal casing. The camera connectors for the power, communication and video signals are located on the right hand side of the board to allow for the camera to be easily connected. The connectors on the left hand side of the board are used to connect to the base station and main motor boards. The RS-485 transceiver is located underneath the board. The connectors located at the bottom of the board are used to deliver $8 \mathrm{~V}$ DC to the LEDs which are connected in series with $121 \Omega$ resistors to minimise the current drawn.

The communication problems were encountered because the RS-485 output TTL signals would not establish communication with the camera which required RS-232 input signals as discussed in Section 4.3.2. Another problem that was encountered was the circuit protection that was required to prevent damage from excess current drawn by the camera and therefore a redesign of the camera board was needed as discussed in Section 6.1.4

\section{Base Station Board}

To enable the user to control the main and camera boards, the computer must connect to the base station board to allow signal transmission to the robot. The initial design of the base station board required two RS-485 chips and five connectors which would be used for power and communication as seen in Figure 6.7.

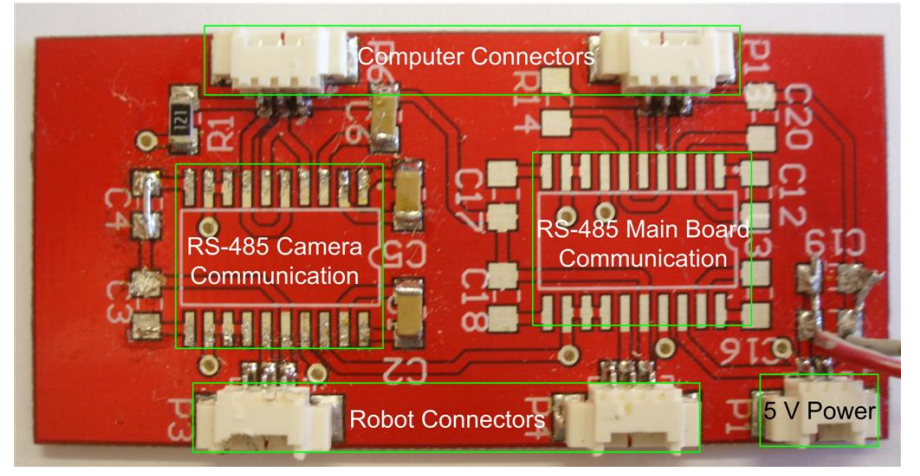

Figure 6.7: Base Station Board Version Three

The reason for inserting two RS-485 transceivers was to enable the com- 
puter to communicate with the camera and the micro-controller simultaneously. This design caused problems because the number of communication lines available along the cable were exceeded which was not desirable. Another issue raised were the returned signals which are generated by the RS-485 chip and therefore another redesign of the board was required as discussed in Section 6.1.4,

\subsubsection{Version Four}

The fourth version of the PCB boards successfully implemented and tested communication between the base station board, main board and the camera board, with adequate circuit protection for the main and camera boards. Each board has been individually populated, tested and debugged to result in a working robot system. The circuit schematics for all three boards are shown in Appendix A.5- A.11.

\section{Main Motor Board}

The fourth revised version of the main board introduced a toggle switch, relay switch, protection fuses and a camera pin header as seen in Figure 6.8 .

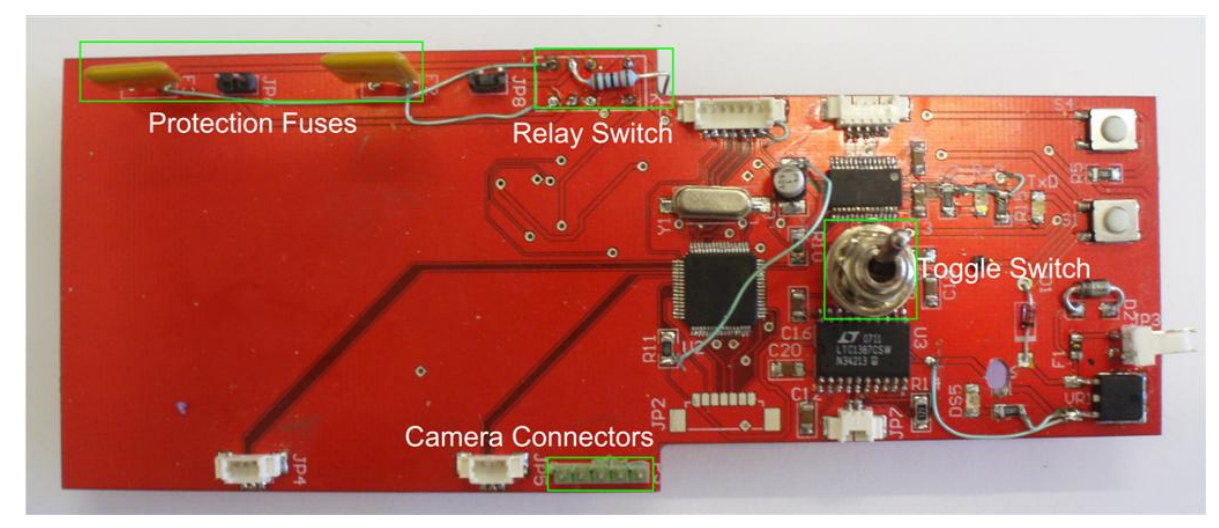

Figure 6.8: Main Motor Board Version Four

The toggle switch is located in the middle of the board to allow the user to select between the micro-controller communication devices as discussed in Section 4.2.2. The switch was chosen in a through hole package 
to provide the user with easy access for on-board reprogramming and testing. The resettable fuses located at the top of the board are used to protect the motor driver boards from being damaged from excess current and are rated at 2.5 A. The resettable fuses are connected to the relay switch enabling the micro-controller to manage the power to the motor drivers as discussed in Section 4.2.1. The camera connectors located at the bottom of the board are used to connect the micro-controller to the camera board. The transmitted signals between the two boards are used to either turn the power on or off to the camera and the communication device as discussed in Section 4.3.2.

\section{Base Station Board}

The second version of the base station board includes a USB connector which allows for a connection to be established between the board and the LabVIEW PC application as shown in Figure 6.9.

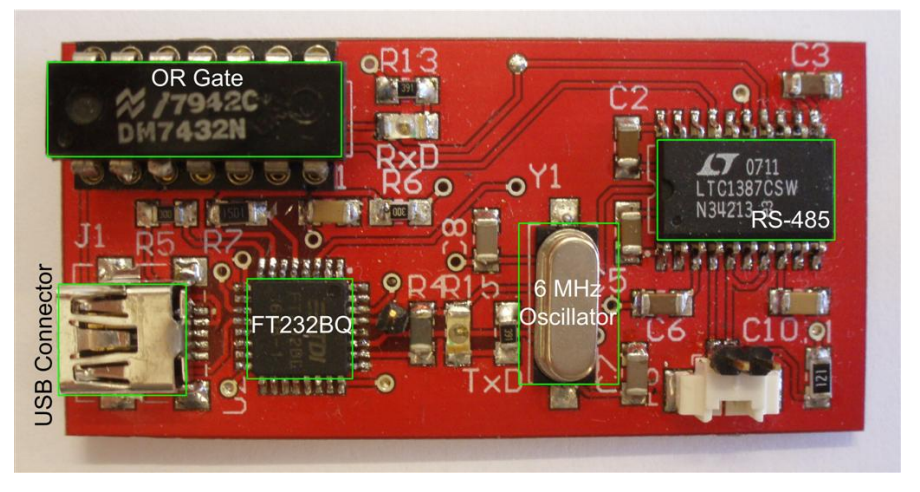

Figure 6.9: Base Station Board Version Four

The USB connector is connected to the FT232BQ chip which is used as a bridging device between the RS- 485 chip and the computer. The FT232BQ chip is inserted because LabVIEW communicates via RS232 signals but the RS-485 chip requires TTL logic signals as discussed in Section 4.4.1. and the $6 \mathrm{MHz}$ oscillator is used to generate the internal clock signal for the chip. The "OR" gate is used to remove signal reflections that occur on the RS-485 transmit and receive lines as discussed in Section 4.4.2. 


\section{Camera Board}

The first version of the camera board encountered problems with the communication device, control and circuit protection. The communication problem was solved by inserting a MAX202 chip which converted the received TTL logic signal levels into RS232 levels which are required for the camera to operate. As shown in Figure 6.10, the MAX202 chip is a through hole package which allows it to be placed on top of the twenty pin socket.

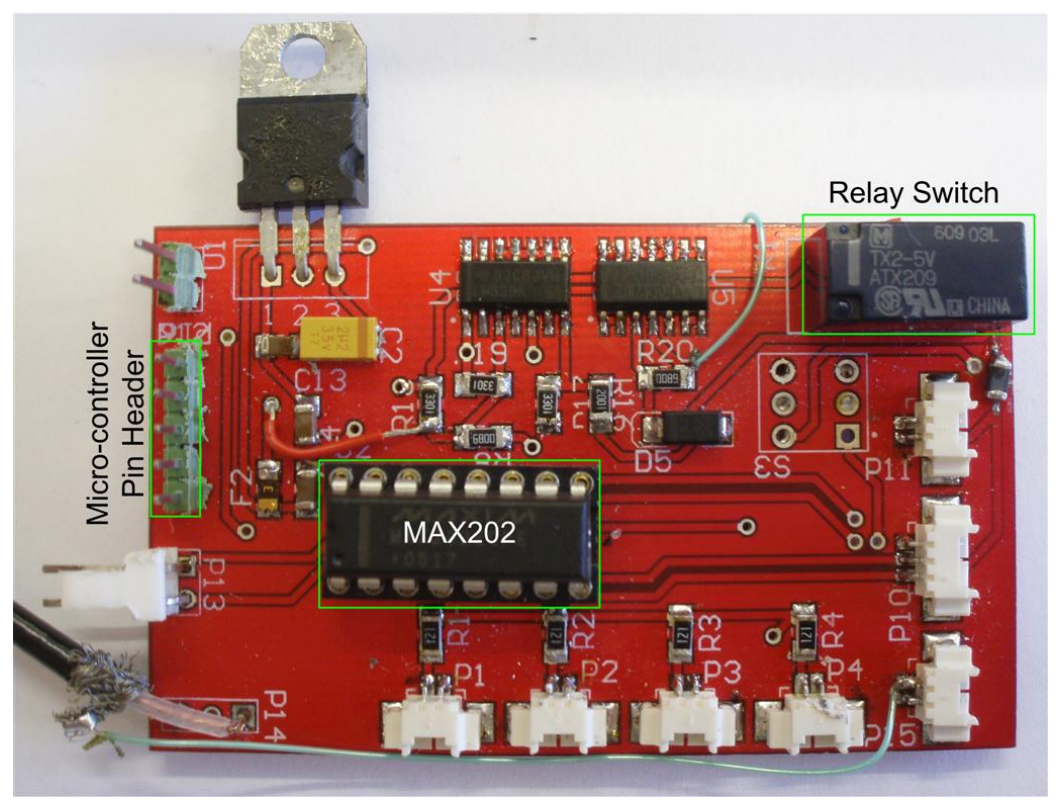

Figure 6.10: Camera Board Version Four

The reason for using this package is because past experience has shown that if the chip breaks down it is more easily replaced with no need for desoldering. The camera circuit protection discussed in Section 4.3.2 requires a relay switch to be inserted which enables the micro-controller to turn the camera and communication devices on or off using the pin header located on the right hand side of the board.

\subsection{Motor Characteristics}

Tests were executed on all three motors without the motor drivers attached to find the power consumed. The reason for excluding the motor drivers 
was to check if the drivers introduced any characteristics into the overall system. The tests were executed by attaching a variable power supply to each individual motor and measuring the current drawn. Figure 6.11 shows the bench mark test that was executed for all three motors under no load conditions.

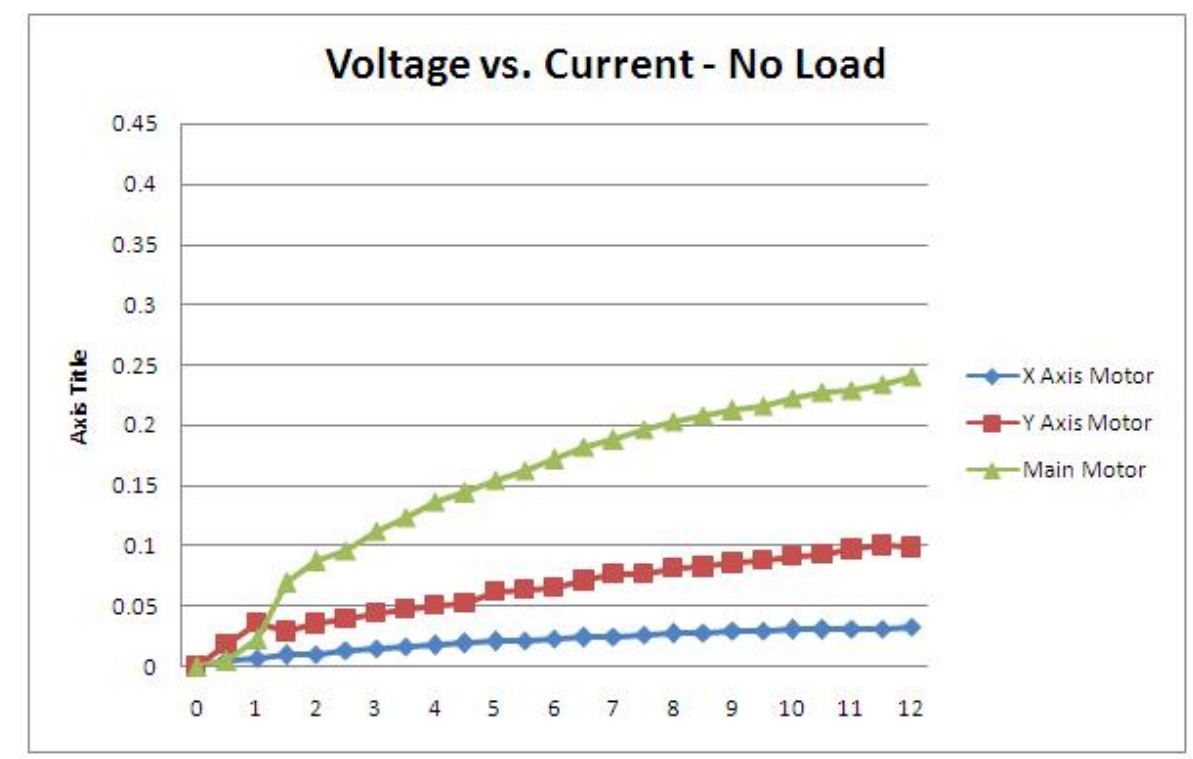

Figure 6.11: Current Drawn - No Load

A linear relationship between the voltage and current is noticed for the $X$ and $Y$ axes motors. The main motor shows a non linear relationship and draws $0.24 \mathrm{~A}$ at maximum voltage. The $X$ and $Y$ axis motors are smaller in size as described in Section 3.3, and draw only 0.033 and 0.098 A respectively.

The same test circuit configuration was repeated for all three motors under normal load which also excluded the motor drivers as shown in Figure 6.12.

The $X$ axis motor retains a linear relationship between the voltage applied and the current drawn and the other two motors show a non linear relationship. The current drawn has doubled for all three motors when subjected to this load. The main motor outputs $4.85 \mathrm{~W}$ when $12 \mathrm{~V}$ is applied across the terminals. The $\mathrm{X}$ axis motor outputs $0.62 \mathrm{~W}$ of power when $12 \mathrm{~V}$ is applied, compared to the $\mathrm{Y}$ axis motor which outputs $2.39 \mathrm{~W}$. 


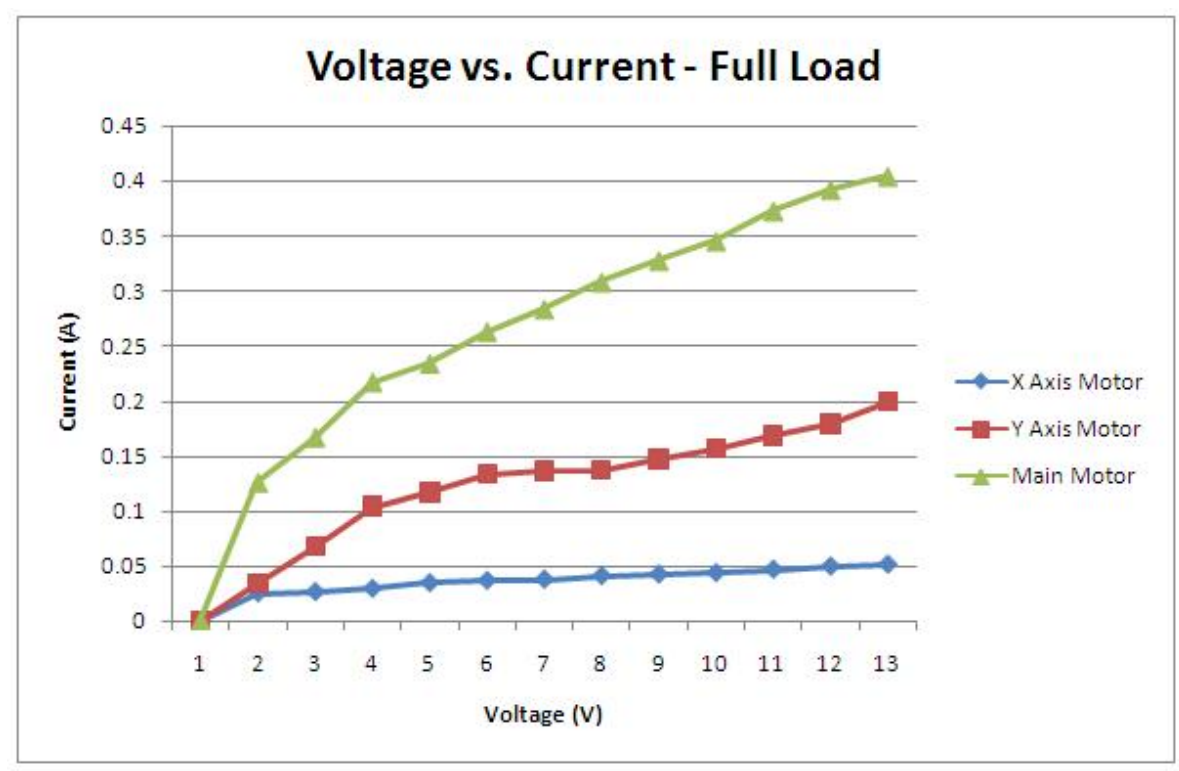

Figure 6.12: Current Drawn - Full Load

This is due to the gearing system that is used along the $\mathrm{X}$ axis (discussed in Section 3.4.1) which does not need as much power to produce movement.

The motors were subjected to a test to measure the number of revolutions that each gear head does in a minute. The same circuit configuration was used as in the previous test and the results are shown in Figure 6.13.

The graph shows that all three motors have a linear relationship between the voltage applied and the RPM. The main motor has the largest gradient due to having a planetary gearing system located inside the gear head. The $X$ and $Y$ axes motors show a smaller gradient relationship due to having smaller gear heads and power ratings.

\subsection{System Characteristics}

As discussed in Section 5.3.2 the motor drivers are set in a stop mode by assigning a value of 76 to the CCAPnH registers. Tests were done to check if the motor drivers introduced any different power characteristics to the overall system. To find the relationship between the two, a number between 15 and 140 is transmitted from LabVIEW to the micro-controller. 


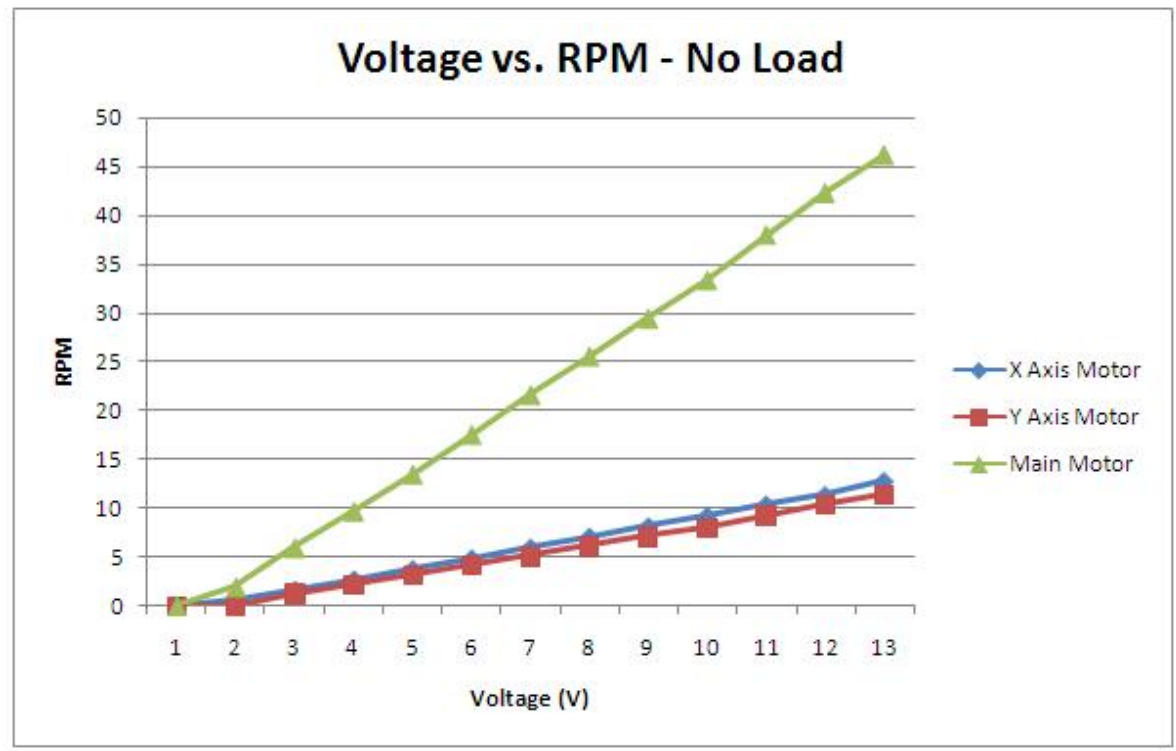

Figure 6.13: Voltage vs. RPM - No Load

The PWM signal produced was measured using an oscilloscope and pulse widths that were produced were recorded and plotted against each other as shown in Figure 6.14 .

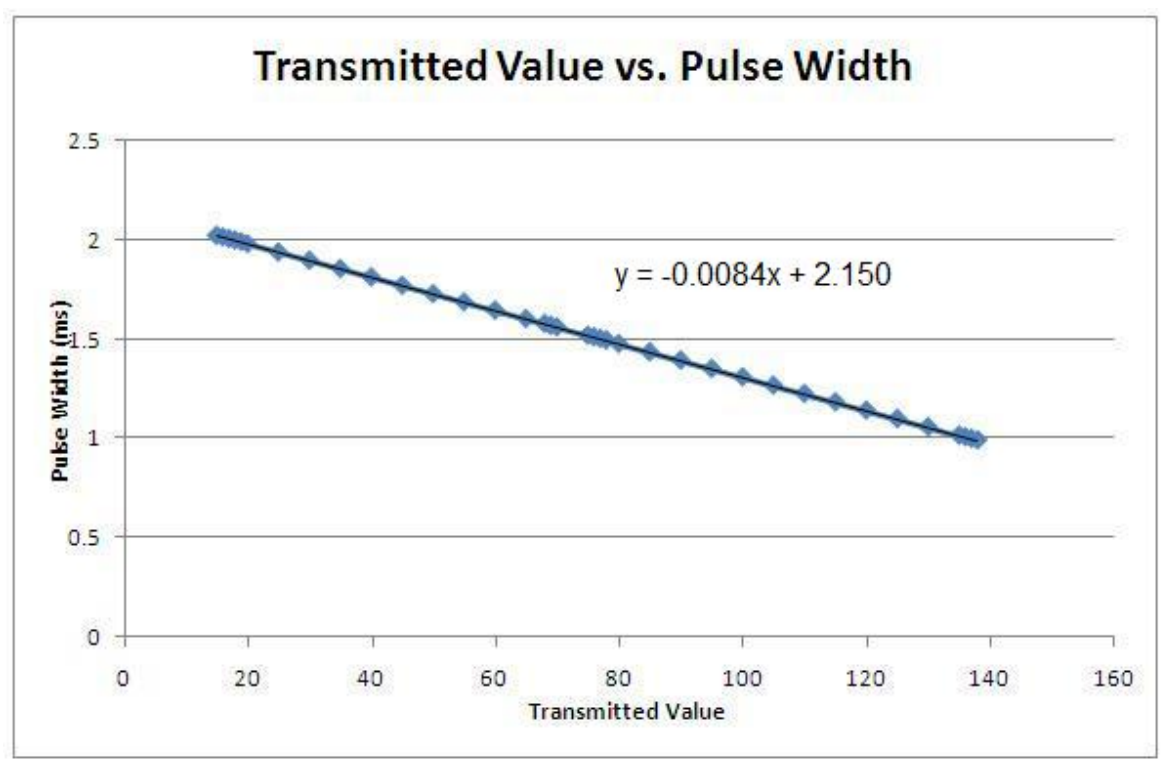

Figure 6.14: Transmitted Value v.s. PWM Pulse Width 
The relationship between the value transmitted and the pulse width is linear. To produce a PWM pulse width of $1 \mathrm{~ms}$ the value transmitted must be set to 137 and for a $2 \mathrm{~ms}$ pulse the value must be set to 18 . A $1.5 \mathrm{~ms}$ pulse width requires a value between 76 and 77 as discussed in Section 5.3 .2 Using Equation 6.1 it is possible to calculate the pulse width of a PWM signal $(y)$ depending on the value transmitted $(x)$ from LabVIEW.

$$
y=-0.0084 x+2.150
$$

The PWM signal transmitted from the micro-controller to the motor driver board creates a DC voltage difference across the motor driver terminals, resulting in a controlled motor movement. A test was done to check the relationship between the transmitted value and the voltage generated across the terminals with no motor attached. The results are shown in Figure 6.15.

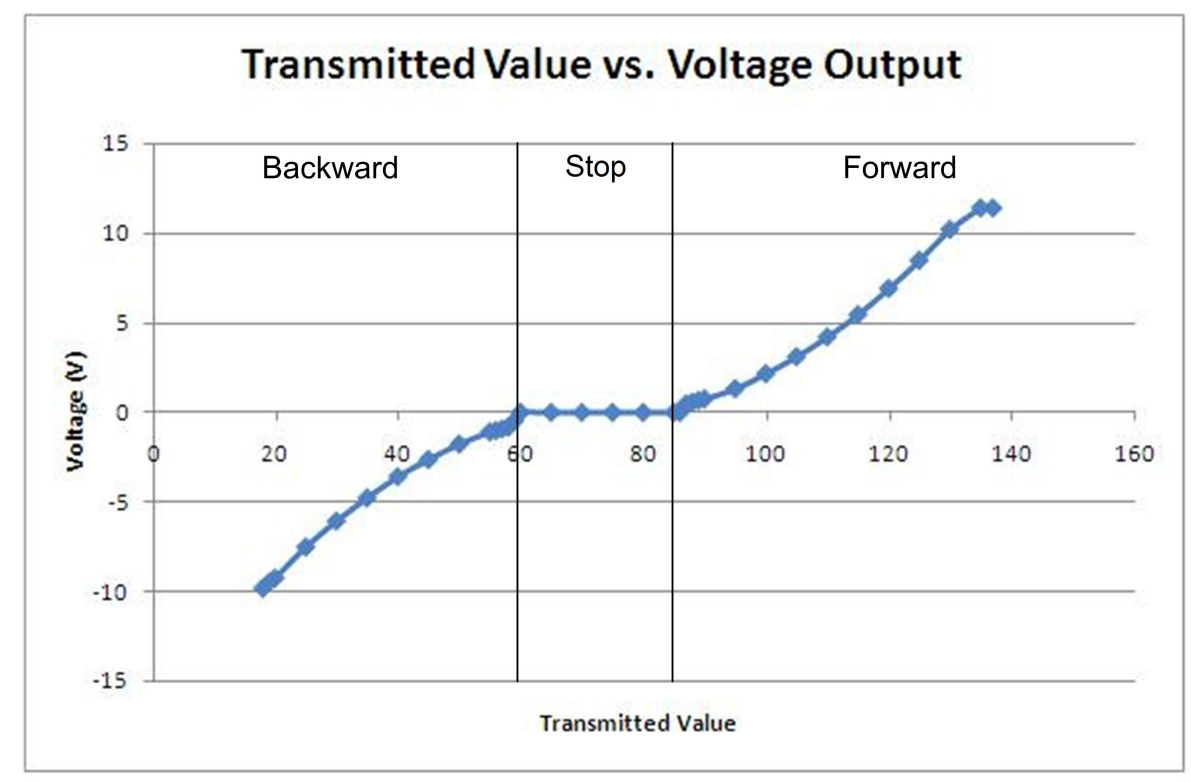

Figure 6.15: Terminal Voltage Difference

When the transmitted value is between 60 and 86 the voltage difference across the terminals is 0 , but as the values increase and decrease on both sides of the scale the voltage difference increases. The maximum voltage recorded is $11.354 \mathrm{~V}$ for a value of 137 and the minimum voltage recorded is $-9.78 \mathrm{~V}$ for a value of 18 . 


\subsubsection{No Load}

The motor drivers were tested to check if any characteristics were introduced to the overall system. The motor drivers were tested under no and normal loads. The no load test was to set a bench mark to establish how much current is being drawn when the robot is in a stationary position and is illustrated in Figure 6.16 .

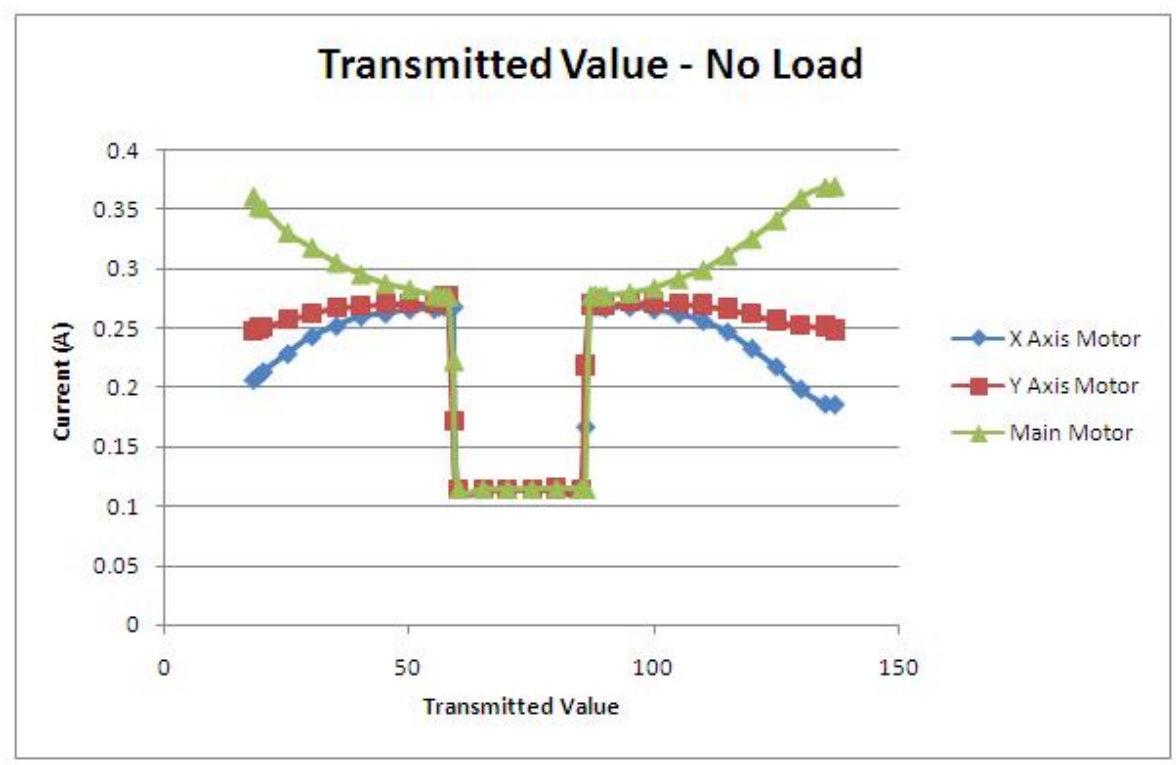

Figure 6.16: Current Drawn - No Load

The big current drop in the middle of the graph is caused by the motor drivers which have a power down mode. If the value received from LabVIEW is between 59 and 86 the motor drivers will remain in the power down mode and the robot will be in stationary position. If the values are gradually increased the motor drivers will produce a voltage difference across the motor terminals and therefore the current drawn increases and the robot will start to move. Under no load the main motor draws $0.37 \mathrm{~A}$ for a value of 137 and $0.36 \mathrm{~A}$ if the value is set to 18 . Similar characteristics are noticed with the $X$ and $Y$ axis motors, where the current drawn is 0.2 and 0.25 A respectively.

A characteristic about the $X$ and $Y$ axis motors can be seen that the current drawn decreases as the speed increases. This is counter-intuitive 
and contradicts the results that were achieved in Section 6.2. The only explanation that can be concluded from such results is that the $X$ and $Y$ axes motors are very non linear. Tests were done to examine the total power consumed by all three motors as shown in Figure 6.17.

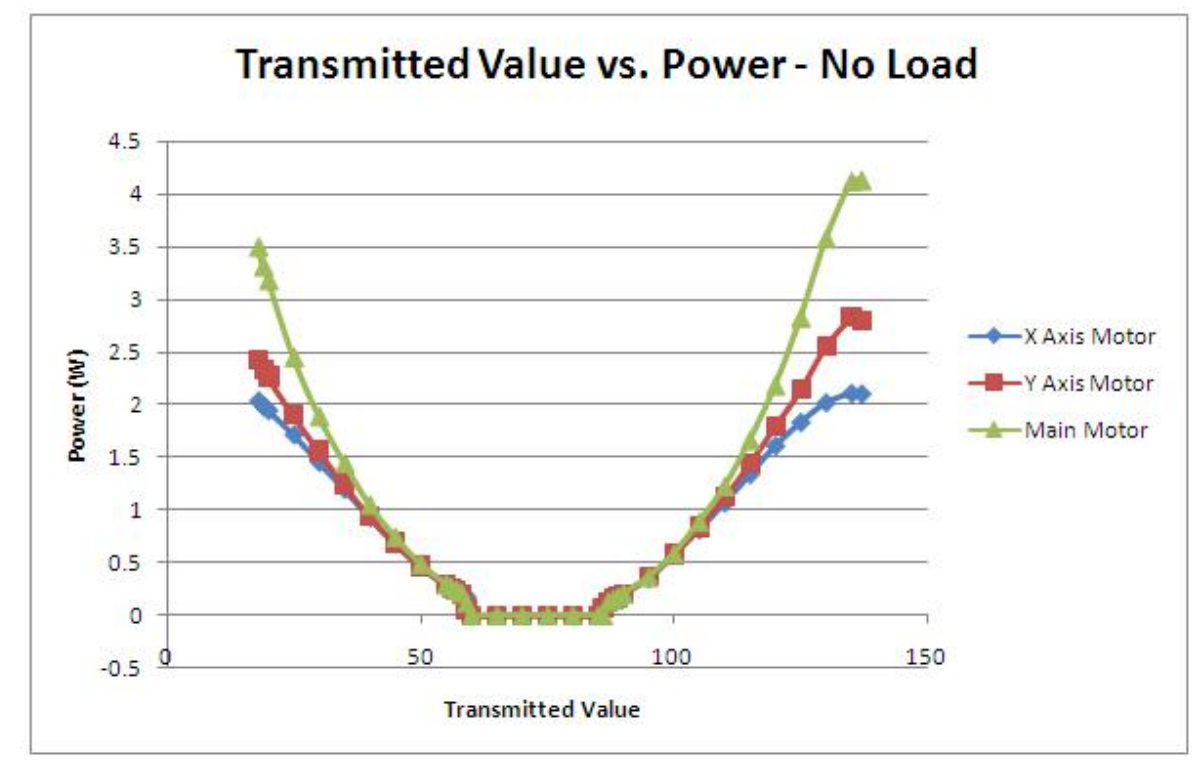

Figure 6.17: Power - No Load

The total power for the $X$ and $Y$ motors is increased as the value transmitted is being changed. This is an expected result because as the voltage difference across the terminals increases so does the total power consumed.

\subsubsection{Full Load}

The same test used for the motor driver boards under no load was repeated when all three motors were under full load. Similar relationships between the transmitted value and the current drawn are produced as shown in Figure 6.18.

In a similar fashion to the experiment with no load, the highest current drawn is by the main motor which was at 0.52 A for a maximum transmitted value of 137 and $0.47 \mathrm{~A}$ for a minimum value of 18 . The $\mathrm{X}$ and $\mathrm{Y}$ axis motors draw 0.21 and $0.36 \mathrm{~A}$ when the maximum value is reached 


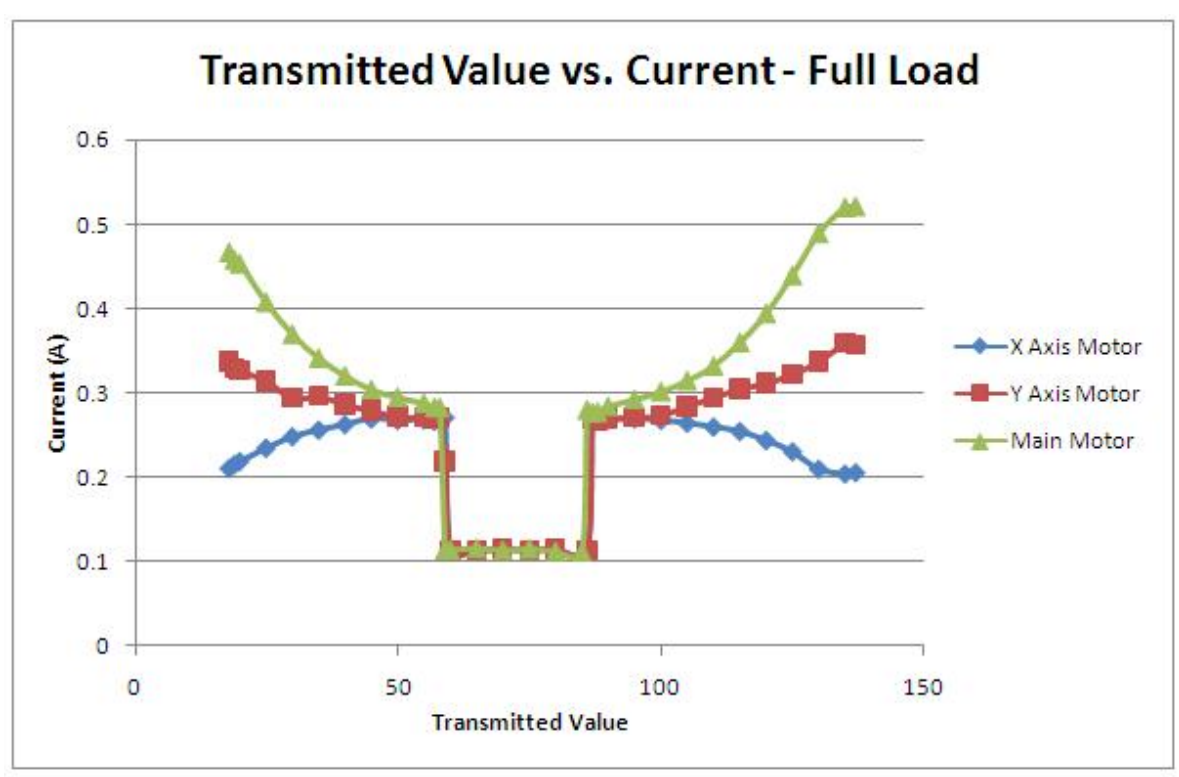

Figure 6.18: Current Drawn - Full Load

respectively and for a minimum value the currents are 0.21 and $0.34 \mathrm{~A}$ respectively. The $X$ axis motor continues to show a very non linear characteristic, with the transmitted value increasing and the current drawn decreasing, but the overall power consumption still increases (Figure 6.19) as expected.

The overall power for all three motors increases with the transmitted value as expected. The main motor outputs $5.75 \mathrm{~W}$ when a value of 137 is reached, compared to the $X$ and $Y$ axis motors which output 2.32 and $3.99 \mathrm{~W}$ respectively.

\subsection{System Performance}

When the robot has been powered and set in stop mode the current drawn is $0.8 \mathrm{~A}$. This current is used to power both motor driver boards, microcontroller, camera and LEDs. The total current consumed when all three motors are at full speed is $1.08 \mathrm{~A}$. If the robot is moving at full speed without any camera head movement the average current drawn is around $0.60 \mathrm{~A}$, but if the robot is in a stationary position with both camera motors 


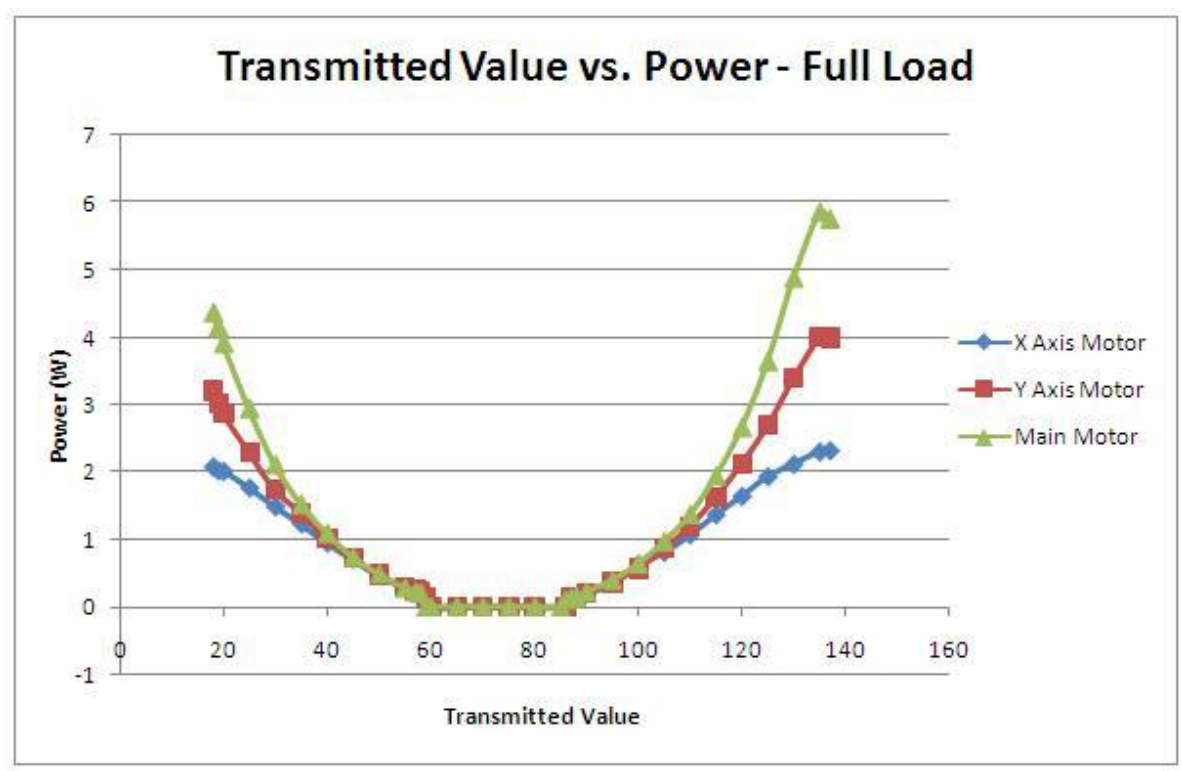

Figure 6.19: Power - Full Load

operating at full speed the current is $0.56 \mathrm{~A}$. Therefore in either case the total current drawn would be around $1.88 \mathrm{~A}$ well below the motor driver board's rating of $5 \mathrm{~A}$.

The total power that is used by all three motors is $12.08 \mathrm{~W}$, but if the robot is in the working environment the power drawn is $6.3 \mathrm{~W}$ for the camera head motors and $5.7 \mathrm{~W}$ for the main motor. The main motor power consumption increases with the distance travelled due to the cable that is being dragged behind it which would get heavier.

The top speed achieved in the forward direction is $245 \mathrm{~m} / \mathrm{h}$ and in the reverse direction the maximum speed is $209 \mathrm{~m} / \mathrm{h}$ as shown in Figure 6.20

The cable used to connect the robot and the computer measured $3 \mathrm{~m}$ in length when tested. The maximum cable length in a working environment is $180 \mathrm{~m}$ and therefore the time required to travel the forward direction would be 44 minutes and in the reverse direction it would be 52 minutes. 


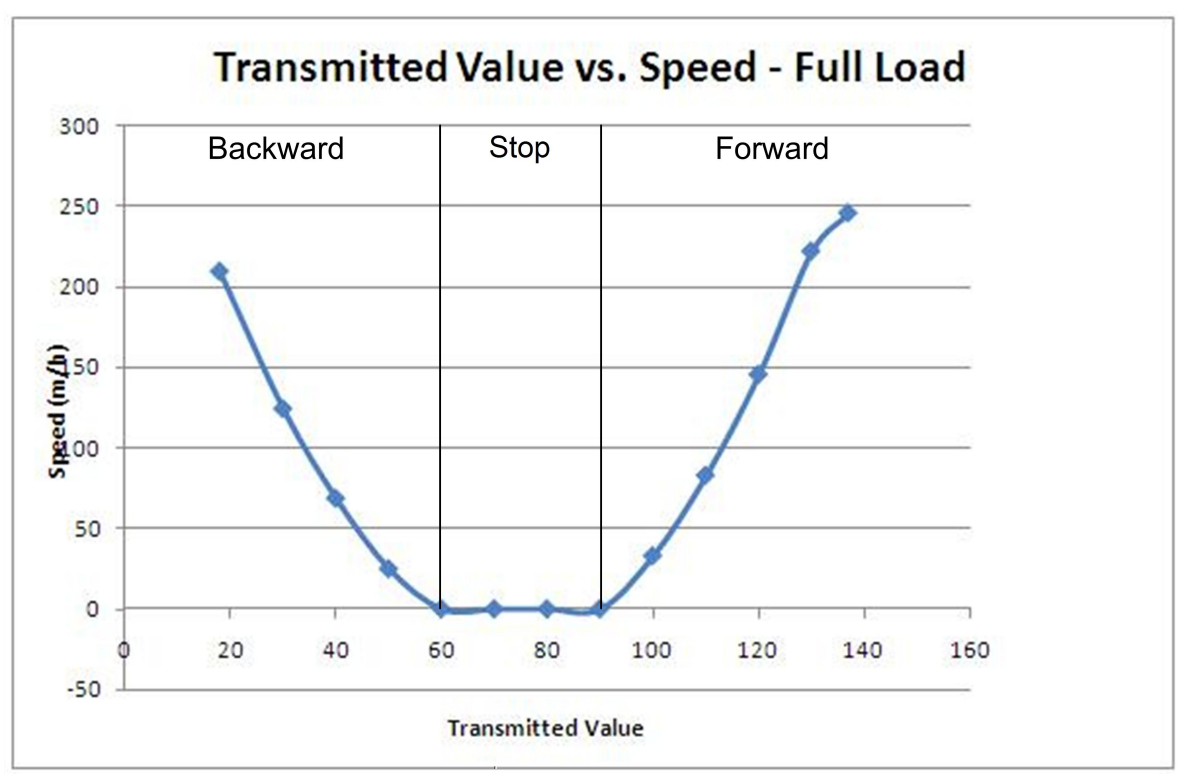

Figure 6.20: Forward and Backward Speed - Full Load 


\section{Chapter 7}

\section{Conclusion}

\subsection{Project Review}

The pipe inspection robot that was received from AES was in a non working condition with no documentation about the design and functionality. The robot's chassis and hardware designs were examined for re-usability and also to gain knowledge on how the robot was originally designed.

The project designed a fully operational pipe inspection robot which is controlled using a LabVIEW graphical user interface from a remotely located computer. The robot is controlled using a main board which controls all three motors (main drive and camera motors), a camera board which controls the camera power and communication, and a base station board which establishes communication between the computer and the robot. The robot is also capable of forwards and backwards movement. The user is able to stop the robot and examine the pipe using the camera head controls located on the graphical interface. The specifications mentioned in Section 1.2 are achieved by integrating the hardware and software designs.

Usability: The graphical user interface allows the user to intuitively operate the robot. Thus no or minimal training is required, increasing the productivity of the operator.

Extensibility: The implemented control via LabVIEW software and electronic boards allows for future development and extensibility. This LabVIEW interface is easily interchangeable which allows for new 
functions to be added or removed. The designed boards allow for expandability if new hardware components were to be added, due to the micro-controller software code being easily reprogrammable.

Generic Application: The hardware and software have been validated throughout several evaluations and the same tasks can be repeatedly executed. The boards designed can be adopted and reused in other platforms which have similar electrical specifications.

Robustness: Extra precautionary measures were taken to minimise hardware and software errors by implementing circuit protection and hard coding user inputs in the user interface.

Reliability: The hardware design is well insulated to reduce the likelihood of any short circuits occuring. If a short circuit does occur, the circuit protection would shut down the faulty part of the board to avoid further damage and inform the user about the problem.

\subsection{Future Work}

\subsubsection{Power Supply}

Problems were encountered with both of the power supplies that were provided by AES. Initially, assumptions were made that both power supplies were in working order. Upon testing the equipment it was discovered that the AES power supplies did not provide the required voltage levels. To solve this problem a new power supply would have to be designed and tested.

The power supply connects to an isolating transformer which has been tested for a $240 \mathrm{~V} \mathrm{AC}$ output. The isolating transformer can be used to deliver power to a $\mathrm{AC}$ to $\mathrm{DC}$ converter, outputting a minimum voltage of $180 \mathrm{~V}$ DC to the DC to DC converter located inside the robot.

This project identified two suitable AC to DC converters that could be purchased such as the Autoranging Rectifier Module (ARM) and the Filter/Autoranging Rectifier Module (FARM) which are manufactured by Vicor Power. Both modules have a auto-ranging line rectification and inrush 
current limiting but the FARM module has a built in EMI (Electromagnetic Interference) filter which filters any noise that might be introduced. The reason for choosing these products is because they are easily integrated with the robot's on-board DC to DC converter. Both modules require an AC voltage input between 180 and $264 \mathrm{~V}$ and they both output a DC voltage between 200 and $370 \mathrm{~V}$ which meets the input voltage specifications for the on-board DC to DC converter. The difference between the two converters is the total power output and the dimensions of the modules. The ARM module measures $57.9 \times 36.8 \times 12.7$ and it can output $1500 \mathrm{~W}$, and the FARM module measures $57.9 \times 55.9 \times 12.7$ and outputs $1000 \mathrm{~W}$ of power (Figure 7.1 [25] \& [26]). The physical measurements and power ratings for both modules are sufficient to be considered for implementation.
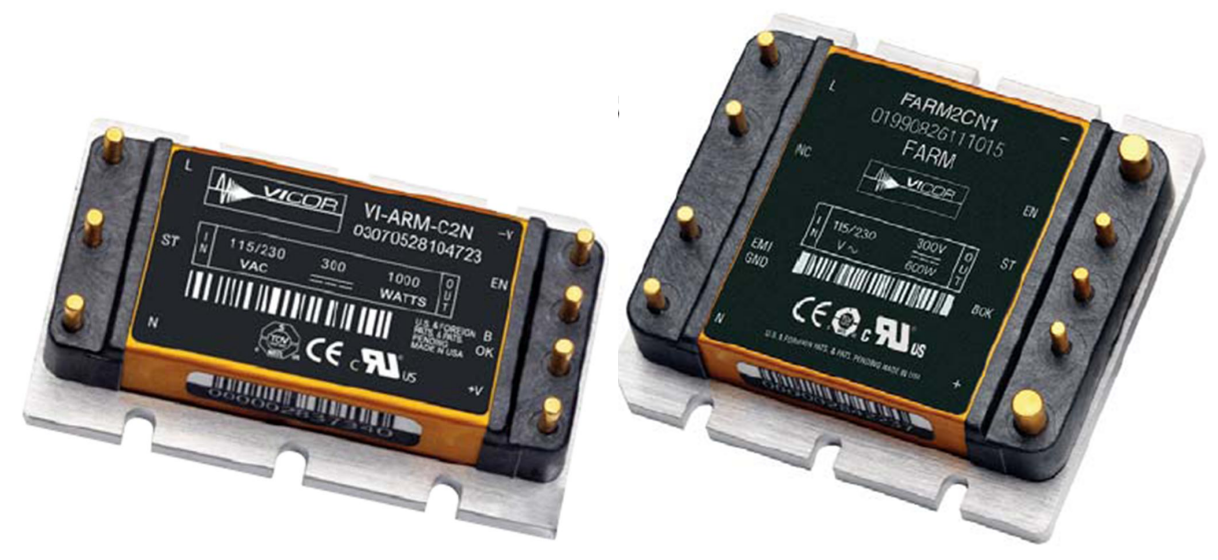

Figure 7.1: Left: ARM Right: FARM

For safety reasons the AC to DC converter should be put into an enclosed casing to prevent the user from touching the high powered terminals. The casing would be constructed out of plastic or insulated metal and it would measure approximately $300 \times 250 \times 100 \mathrm{~mm}$. The casing could also contain the base station board, video capture card and pin connectors that would connect to the robot, transformer and computer.

\subsubsection{Motor Encoders}

The motor encoders (discussed in Section 3.5) located inside the robot are in working order and the manufactured boards have been designed to 
receive the encoder signals. The micro-controller is capable of receiving these signals but the software is required to be written and implemented to process the data.

The motor encoder signals are connected to the micro-controller on pins 4 and 5 of port 1 . The signal would be captured using the CCAPM register to calculate how many degrees the encoder has rotated. This calculation would be stored inside a global variable to be transmitted back to the base station, informing the user if the maximum or minimum limits of the camera head have been exceeded. The operator would use this information to either correct the error or completely shut down the robot if needed.

\subsubsection{Internal Camera Enclosure}

The camera board that has been constructed and implemented is too large to be placed inside the camera head's enclosure. The possible solutions considered were to either cut and trim down the camera board or drill out the excess plastic inside the enclosure. Neither considerations would achieve an optimum result and therefore a redesign of the board would have to be done. The new board would measure less than or equal to $34 \times$ $65 \mathrm{~mm}$ and it would require for new, smaller surface mount components such as the relay switch and the MAX202 chip. The voltage regulator that is currently used occupies a large surface area inside the enclosure which is required to dissipate the heat generated and thus that component size has to remain the same.

The internal enclosure of the camera and the robot would have to be sealed to prevent any moisture entering from the outside environment. The previous robot removed this problem by applying a plastic sealant between the gaps. The same or similar approach could be taken for this project to minimise any moisture that might enter the robot.

\subsubsection{Cable Reel}

The cable reel that is currently used transmits a signal back to the power box containing information about the distance that the robot has travelled. 
The documentation about how the electronics operate inside the reel or what signals are required to gather information does not exist.

A possible solution would be to reverse engineer the electronics located inside the reel. This approach would require time to debug the current design and to select the new electronic components to be purchased. A different approach would be to fully discard the current design and construct new and better electronics.

There are two types of cable reels that can be constructed. The first type is a manual reel which requires user intervention. The robot would travel in a forwards direction and the cable would leave the reel at a rate which would depend on the speed that the robot was travelling at. The user intervention is needed when the robot is travelling backwards and the cable has to be put back on the reel. This is a delicate process because the user has to pull the cable at the same rate as the robot is travelling at. If the user pulls too fast the robot could flip and the user would have to drag it out, but if the user is too slow the robot would run over the cable and end up being tangled up which would also require for it to be dragged out.

The second type is a motorised reel which rolls the cable out and in at the same rate as the robot is travelling at. This type of reel is preferred because it removes the need for user intervention. The negative aspect is that the motors eventually break down due to wear and tear over time and in the past they had to be replaced which is a costly and time consuming process. The current motorised reel supplied by AES is shown in Figure 7.2 .

\subsection{Summary}

The robot's software control and hardware have been designed to provide full functionality using standard electronic components and to facilitate future development. The electrical and mechanical designs can be adopted to fit/control larger or smaller scale robots, so that they can be reproduced on a larger scale. The final robot design meets all of the initial project specifications, is robust and expandable. This is an excellent 


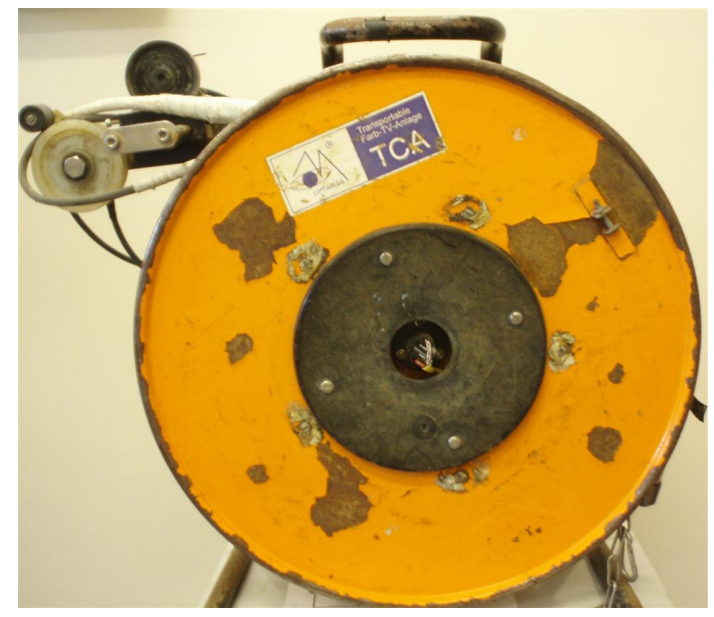

Figure 7.2: Motorised Reel

working system to initiate AES's robot manufacturing goals. 


\section{Bibliography}

[1] RoboProbe Technologies Inc, Home Page, www.roboprobe.com, visited on $05 / 09 / 2007$.

[2] Electromechanica Inc, Home Page, http://www. electromechanica.com/index.html, visited on 11/09/2007.

[3] GE Inspection Technologies, Home Page, http://www. geinspectiontechnologies.com, visited on 15/07/2008.

[4] Radiodetection, Home Page, http://www.radiodetection. com/, visited on 15/07/2008.

[5] Triventek A/S, Home Page, http://www.triventek.com/, visited on $15 / 07 / 2008$.

[6] Angelus Research Coporation, Product Home Page, http://www. angelusresearch.com/products.htm, visited on 11/09/2007.

[7] RoboProbe Technologies Inc, Mole Crawler, http://www. roboprobe.com/catalog.aspx?productid=15, visited on 07/01/2009.

[8] RoboProbe Technologies Inc, Mole Crawler, http://www. roboprobe.com/images/100/IM-Crw-Mole_006.jpg, visited on $07 / 01 / 2009$.

[9] RoboProbe Technologies Inc, Gopher Crawler, http://www. roboprobe.com/catalog.aspx?productid=14, visited on 07/01/2009. 
[10] RoboProbe Technologies Inc, Gopher Crawler, http://www . roboprobe.com/images/100/IM-Crw-Gopher_016.jpg, visited on $07 / 01 / 2009$.

[11] RoboProbe Technologies Inc, Wolverine Crawler, http: //www.roboprobe.com/catalog.aspx?productid=19, visited on $07 / 01 / 2009$.

[12] RoboProbe Technologies Inc, Wolverine Crawler, http://www. roboprobe.com/images/100/IM-Crw-Wolverine_008.jpg, visited on $07 / 01 / 2009$.

[13] GE Inspection Technologies, Rovver 600, http:// geinspectiontechnologies.com/images/rvi/rovver/ main-rovver600-robotic-crawler.jpg, visited on 07/01/2009.

[14] Faulhaber Group, Home Page, http://www. faulhaber-group. com, visited on $08 / 04 / 2008$.

[15] ETA Electric Industry Co.Ltd, Home Page, http://www . eta-usa . com, visited on 08/04/2008.

[16] SCANCON Scandinavian Construction Company, Product Page, http://www.scancon.dk/Mini_Shaft_incremental_ Encoders.htm, visited on 14/04/2008.

[17] Atmel Corporation, Home Page, http://www . atmel.com, visited on $18 / 04 / 2008$.

[18] Dimension Engineering, http://www.dimensionengineering • com, visited on 04/09/2007.

[19] Vicor Power, Home Page, http://www . vicorpower.com, visited on $28 / 04 / 2008$.

[20] RS485 quick reference, Home Page, http://www.rs485.com/ rs 485 spec.html, visited on 11/09/2007. 
[21] Total Turnkey Solutions, Home Page, http://www . turnkey-solutions.com.au, visited on 05/09/2007.

[22] Dimension Engineering, Photograph, http://www. dimensionengineering.com/images/products/ Sabertooth2X5big.jpg, visited on 30/03/2009.

[23] Vishay Electronics, Home Page, http://www.vishay.com/, visited on 06/09/2008.

[24] AVerMedia, Home Page, http://www.avermedia.com/, visited on $08 / 07 / 2008$.

[25] Vicor Power, Specifications Sheet, http://www.vicr.com/ products/filter/arm/, visited on 06/09/2008.

[26] Vicor Power, Specifications Sheet, http://www.vicr.com/ products/filter/farm/, visited on 06/09/2008. 


\title{
Glossary
}

\author{
ACW Anti Clockwise, 35 \\ ADC Analogue to Digital Converter, 11 \\ AES Associated Environmental Services, 2 \\ ARM Autoranging Rectifier Module, 100 \\ CCAPM PCA Compare/Capture Module Mode, 73 \\ CMOD PCA Counter Mode Register, 74 \\ CW Clockwise, 35 \\ DTR Data Terminal Ready, 48 \\ ECOM Enable Compare Mode Module, 73 \\ EMI Electromagnetic Interference, 100 \\ FARM Filter/Autoranging Rectifier Module, 100 \\ IEN0 Interrupt Enable Register, 73 \\ LEDs Light Emitting Diodes, 51 \\ MOSFET Metal Oxide Semiconductor Field Effect \\ Transistor, 14 \\ PAL Programmable Array Logic, 11 \\ PCA Programmable Counter Array, 41 \\ PCON Power Control Register, 64
}




$\begin{array}{ll}\text { PIR } & \text { Pipe Inspection Robot, } \\ \text { PWM } & \text { Pulse Width Modulation, } 11 \\ \text { RAM } & \text { Random Access Memory, } 11 \\ \text { ROM } & \text { Read Only Memory, 11 } \\ \text { RTS } & \text { Request To Send, } 48\end{array}$


Appendices 
Appendix A

\title{
Hardware
}

\author{
A.1 Parts
}

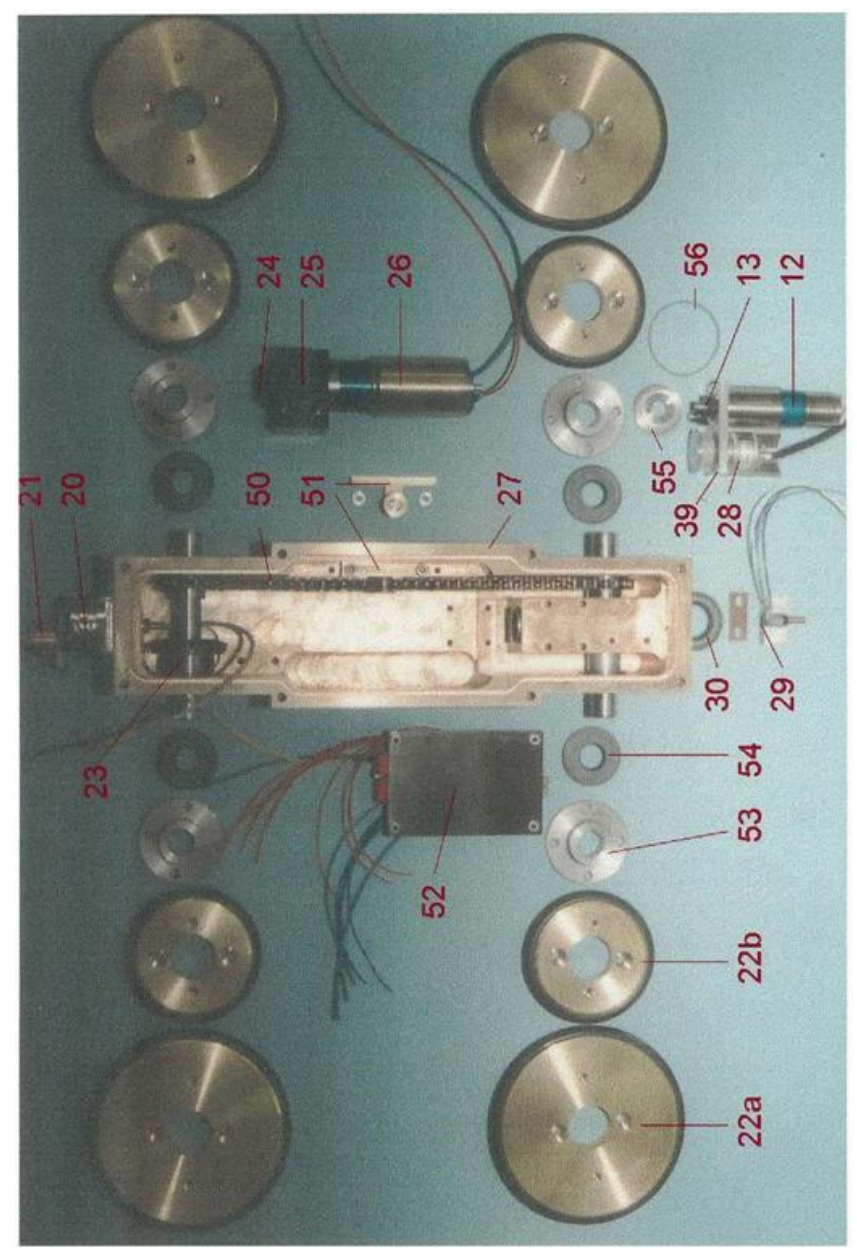




\section{A.2 Parts List One}

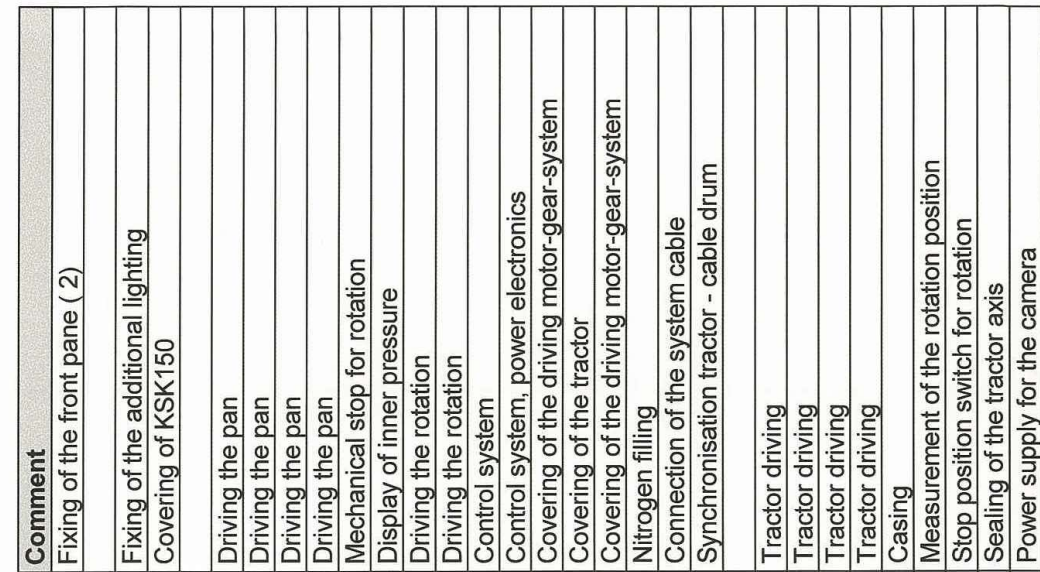

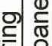
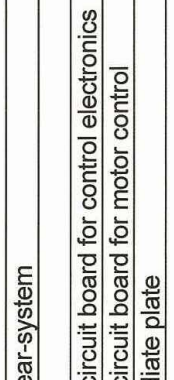

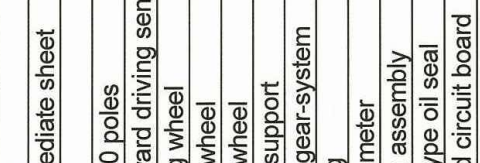

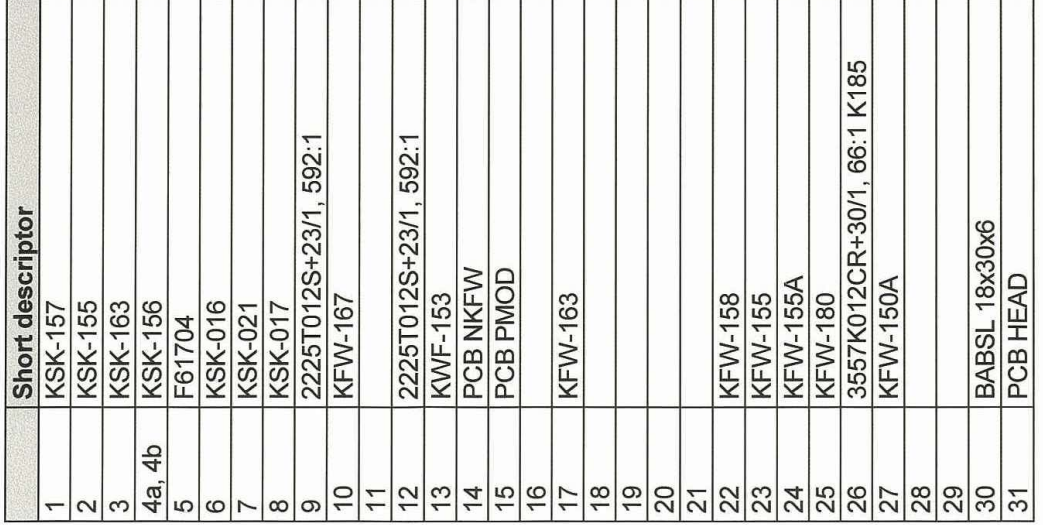




\section{A.3 Parts List Two}
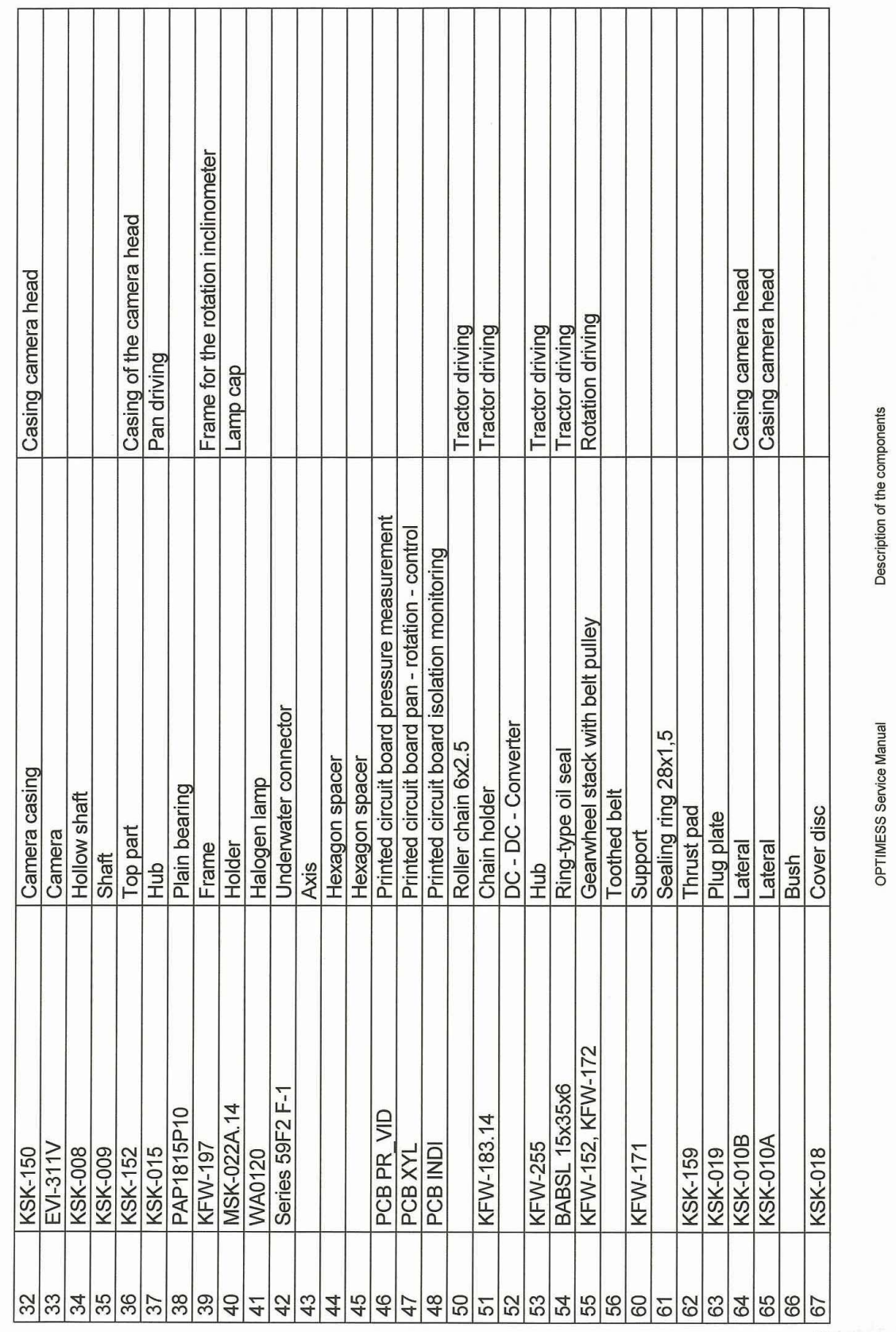


\section{A.4 Parts List Three}

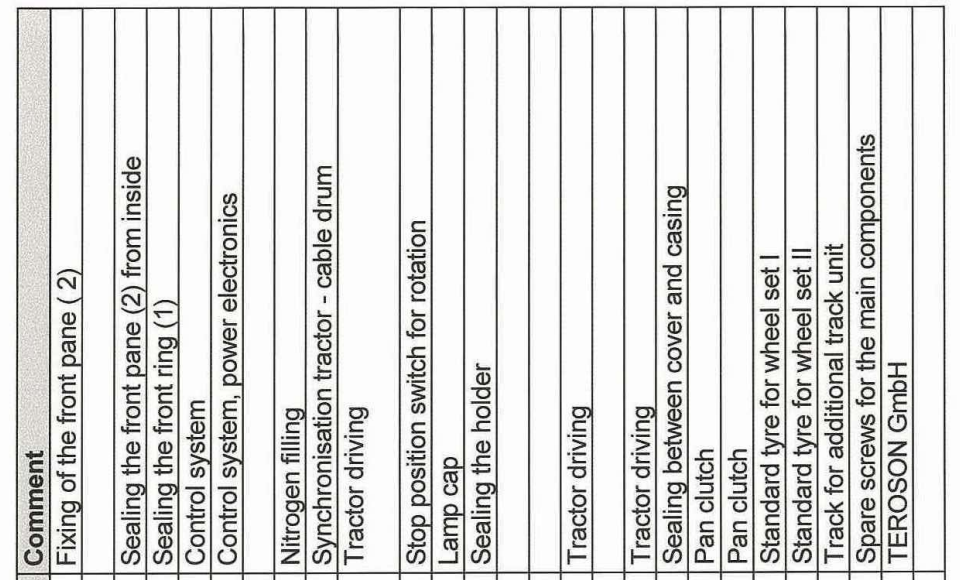

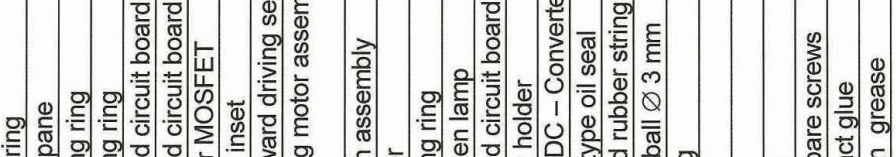

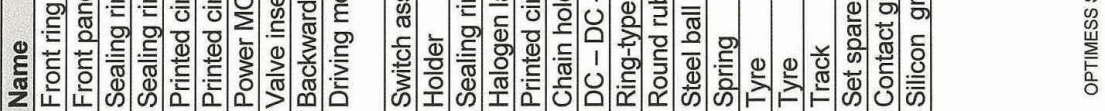

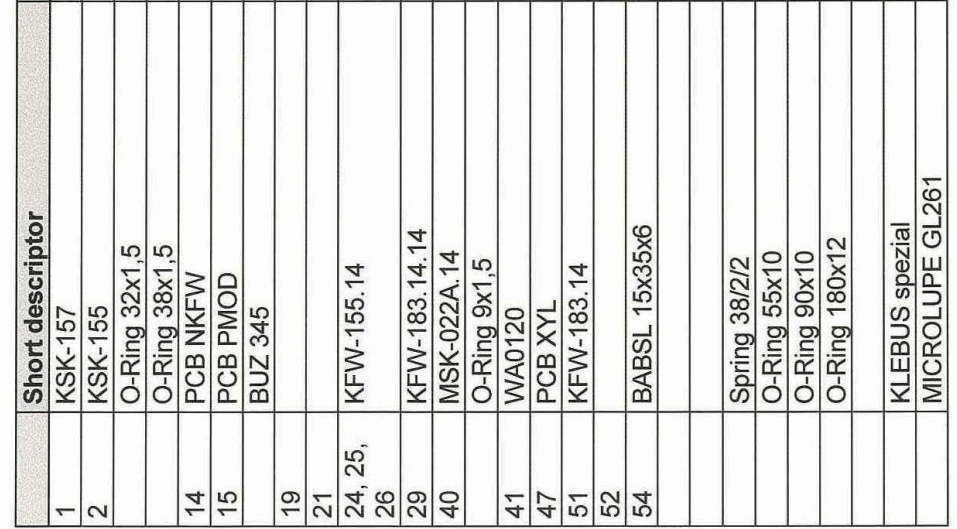




\section{A.5 Main Board Micro-controller}

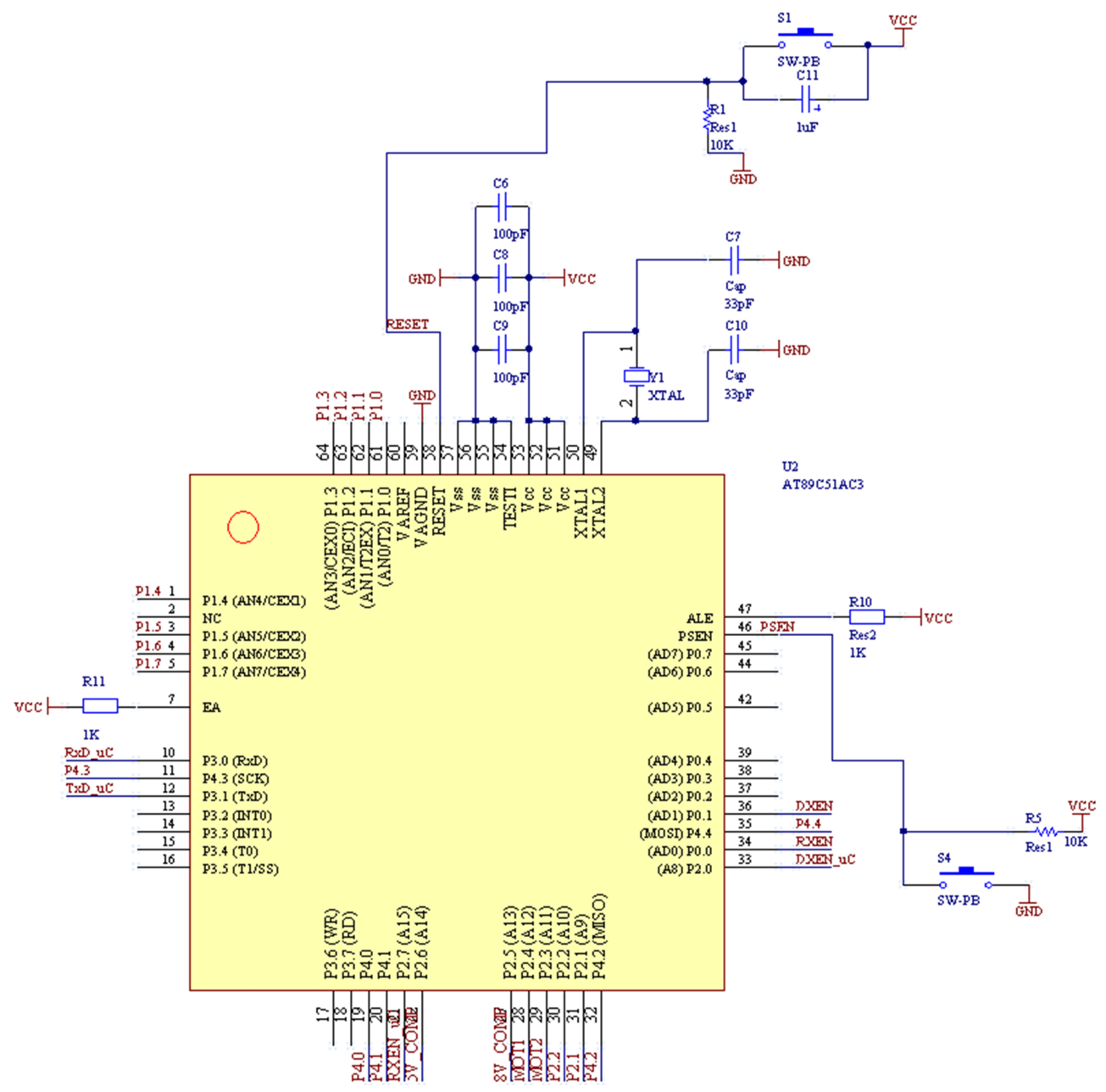




\section{A.6 Main Board Power Supply}

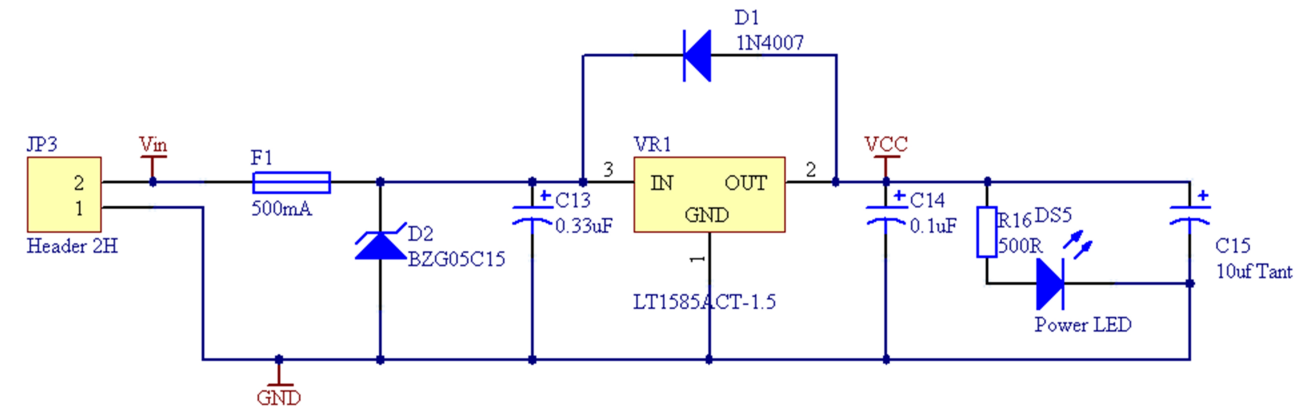

\section{A.7 Main Board SP3238}

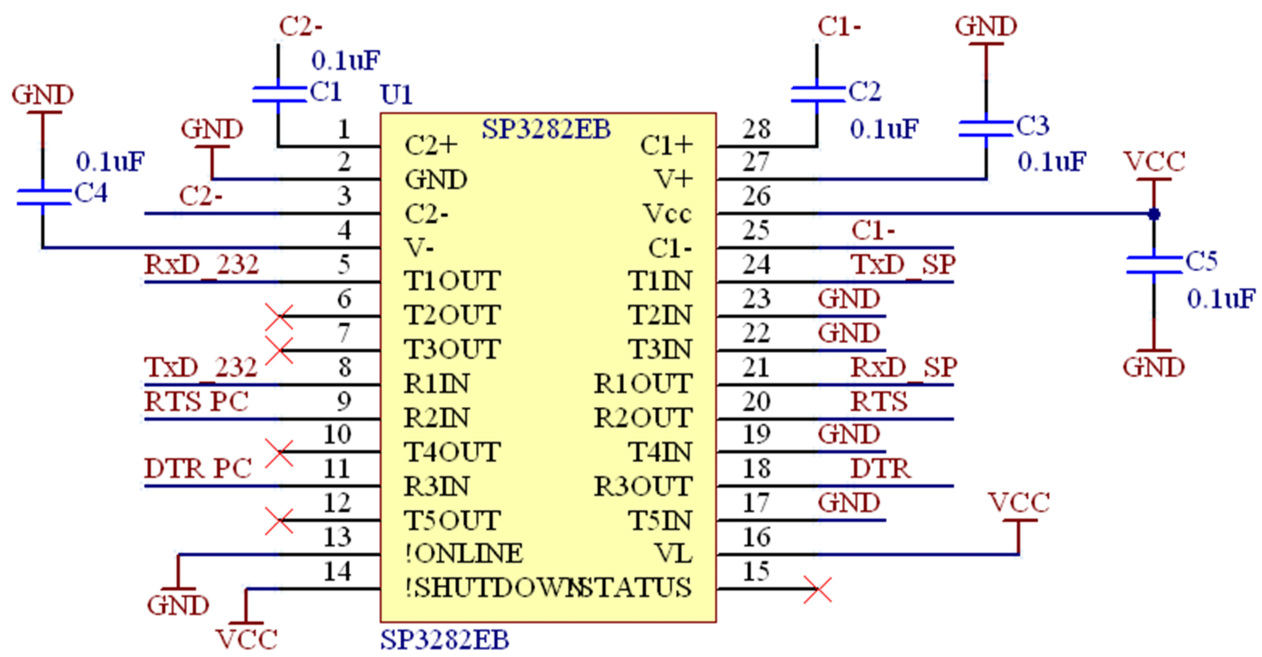




\section{A.8 Main Board RS-485}

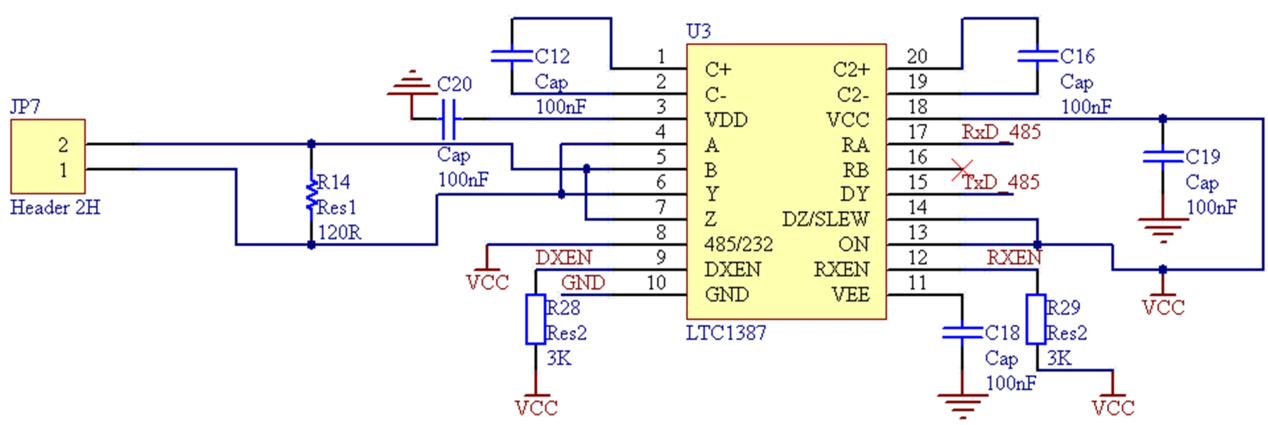

\section{A.9 Main Board Circuit Protection}
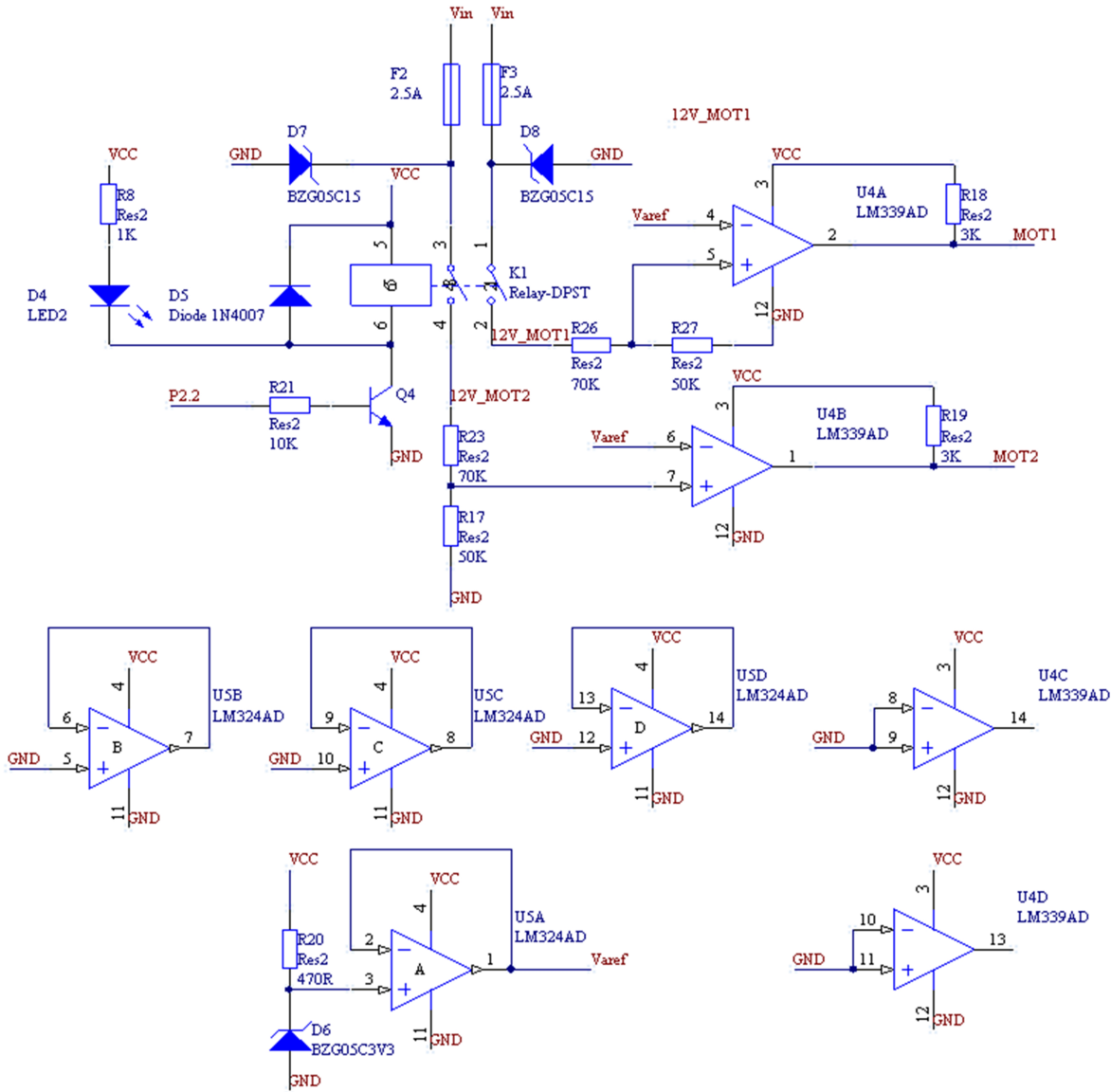


\section{A.10 Camera Board}

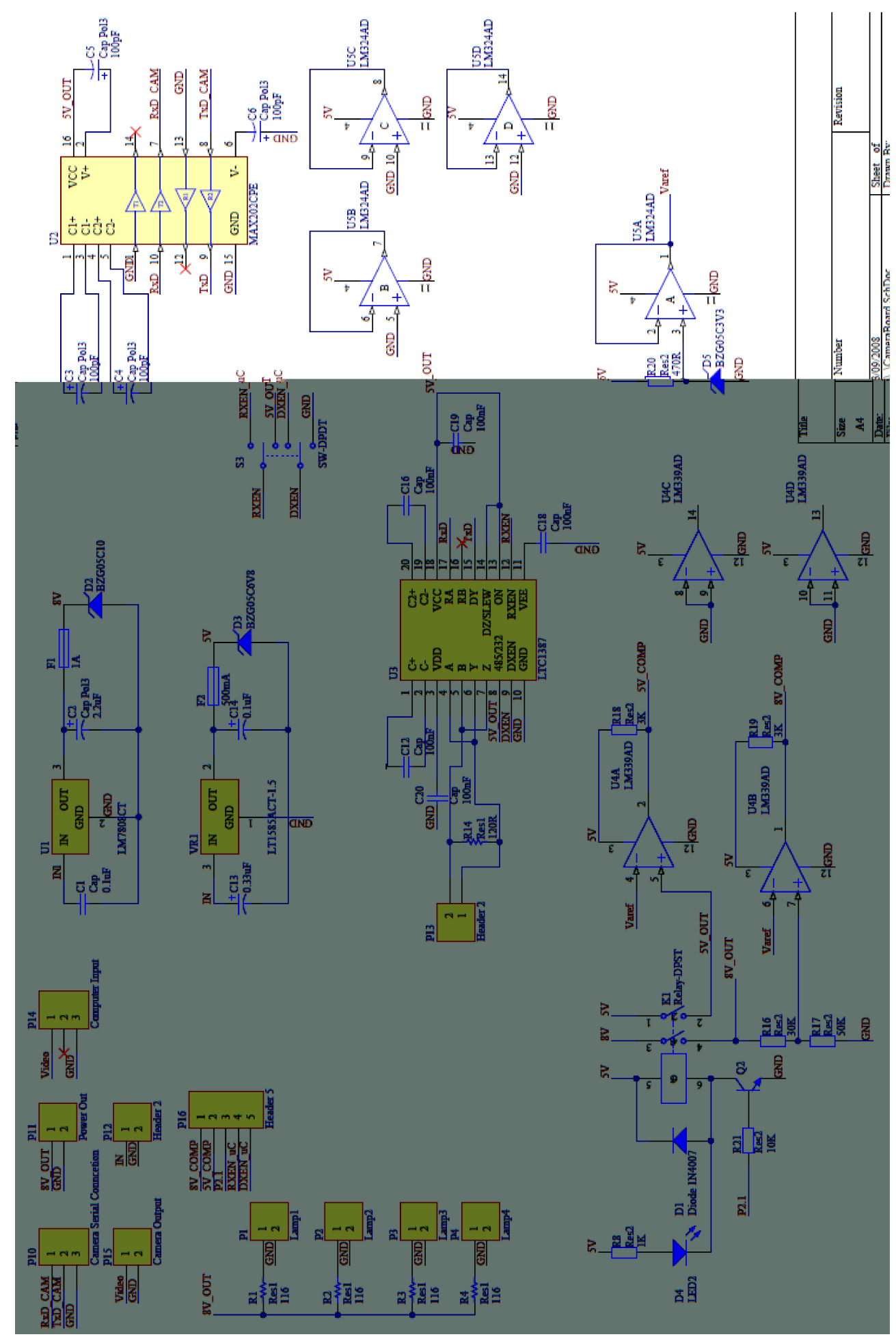




\section{A.11 Base Station Board}

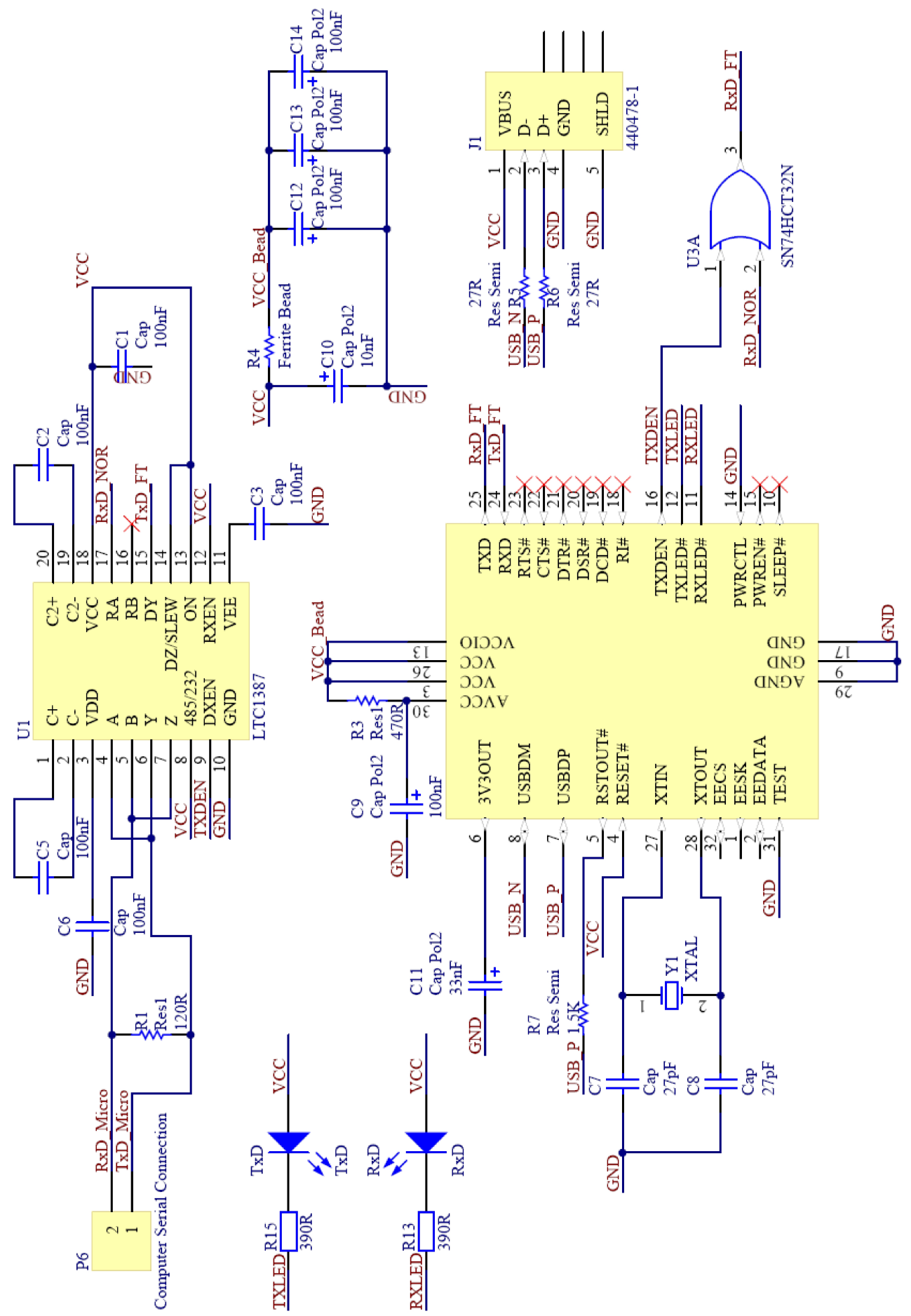


Appendix B

Software 


\section{B.1 Micro-controller Configuartion}

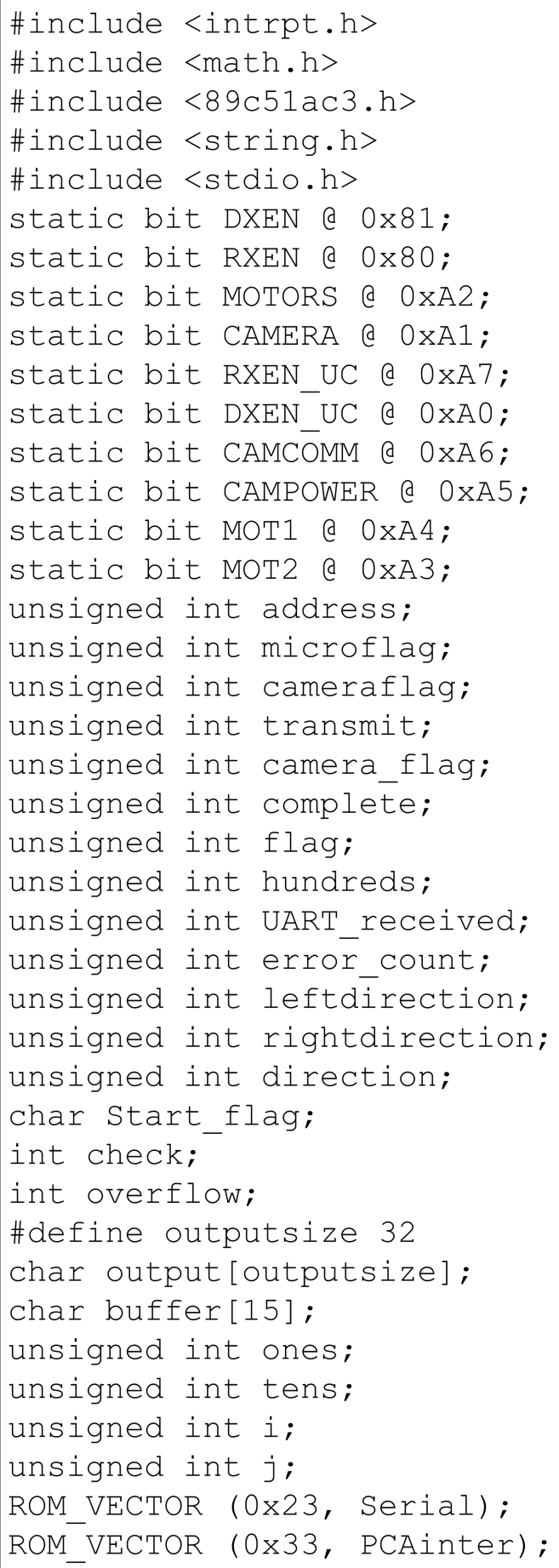




\section{B.2 Micro-controller ASCII To Decimal}

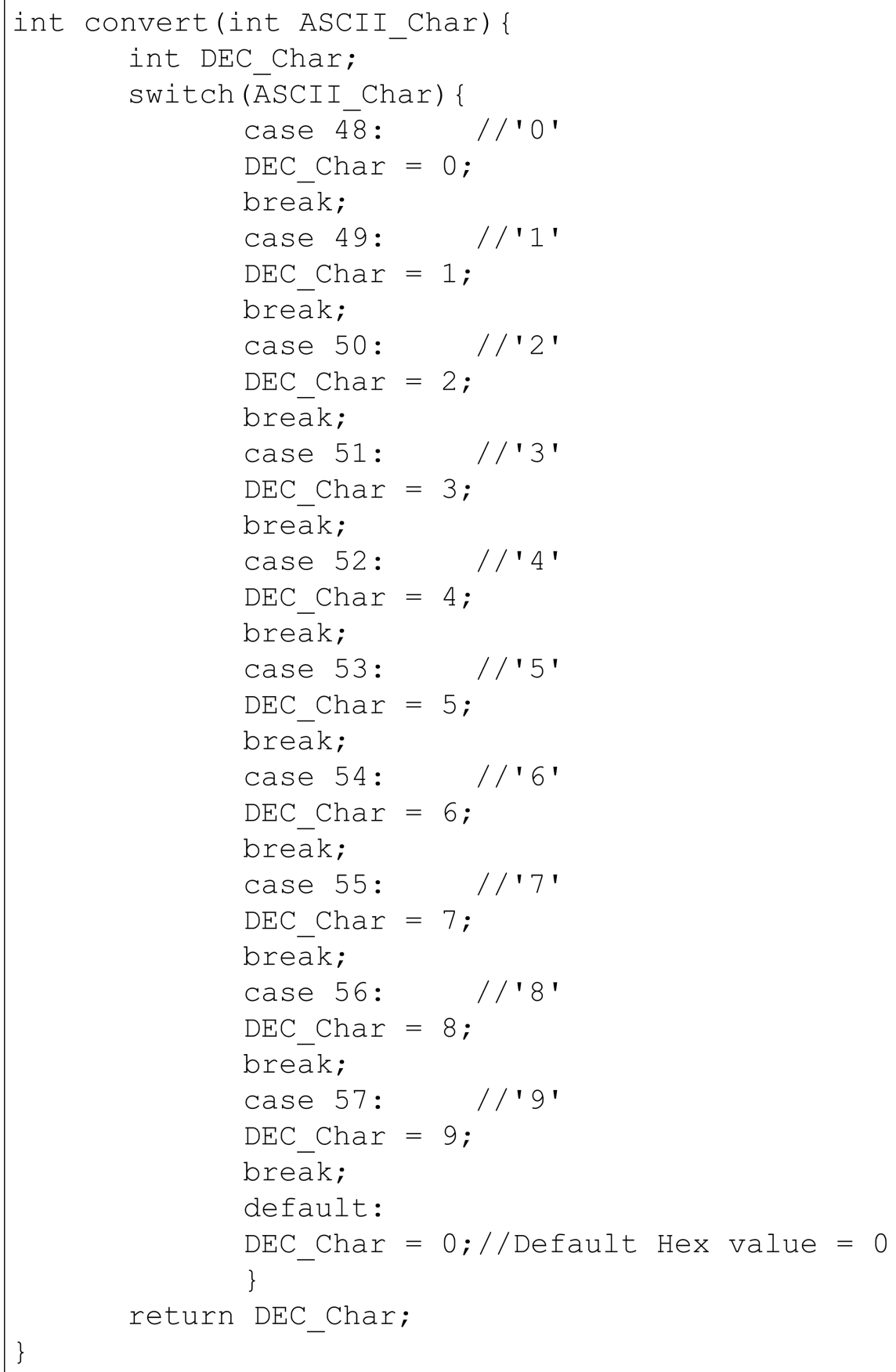




\section{B.3 Micro-controller ASCII To Hexadecimal 1}

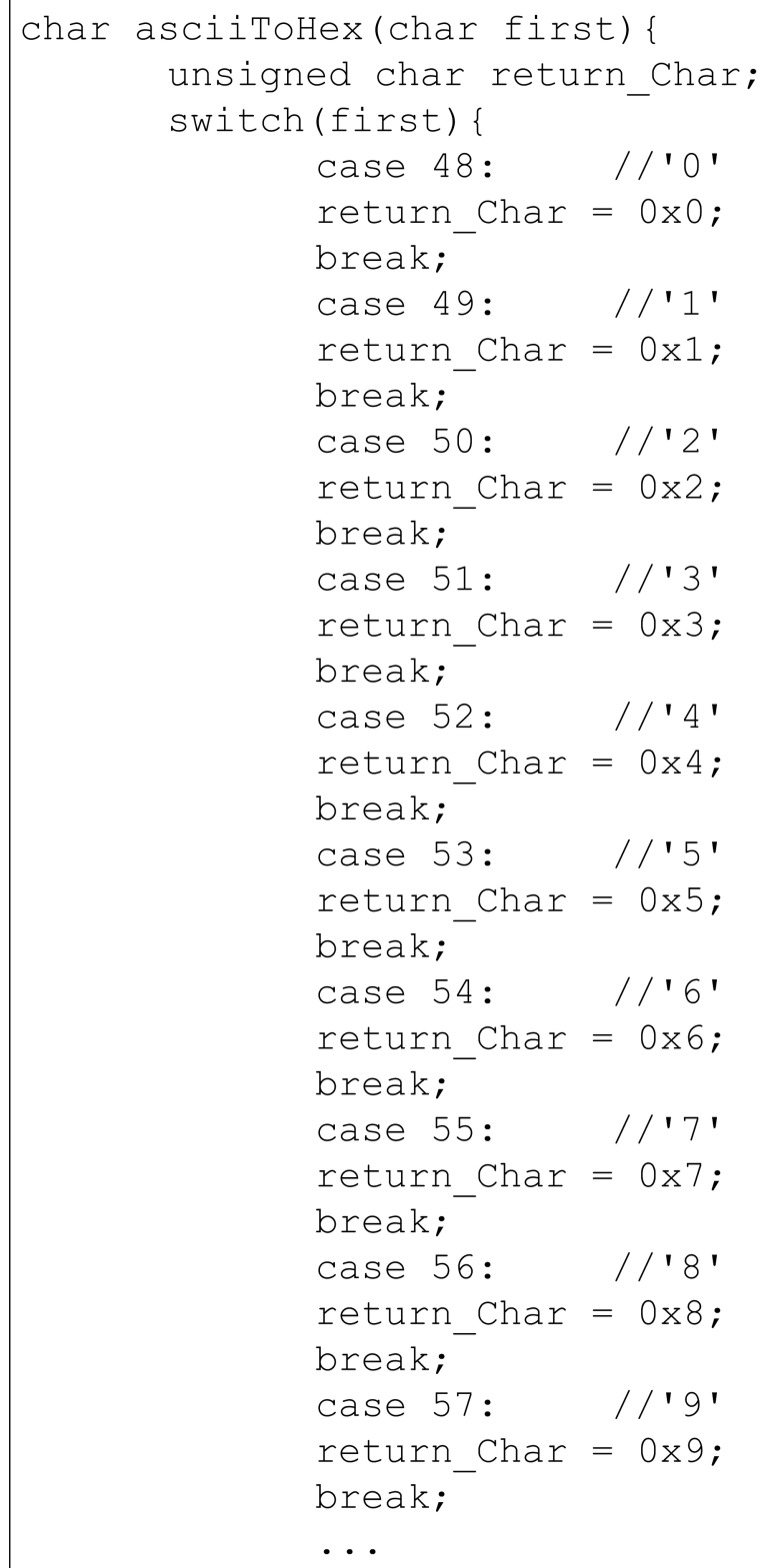




\section{B.4 Micro-controller ASCII To Hexadecimal 2}

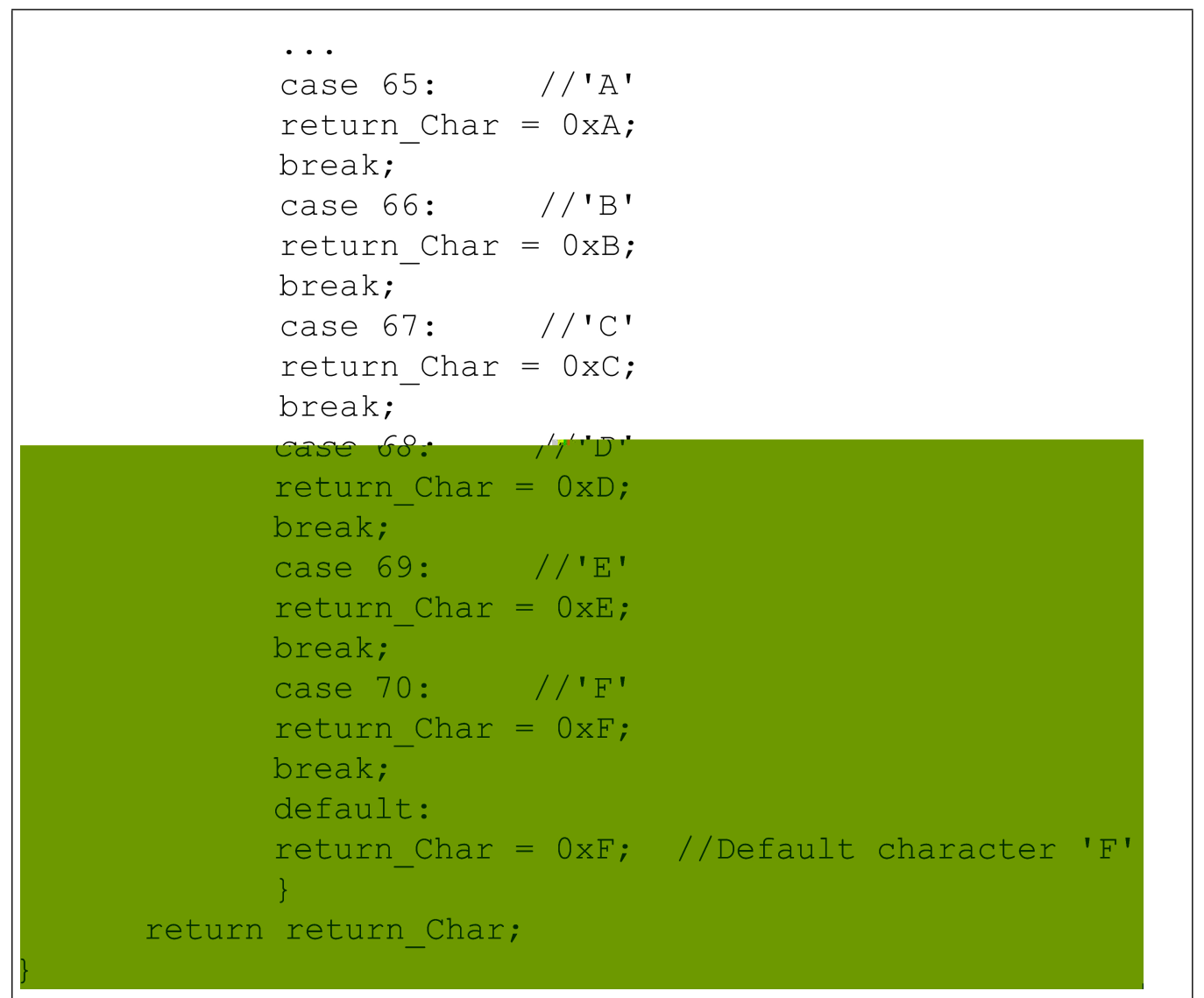




\section{B.5 Micro-controller Serial Interrupt}

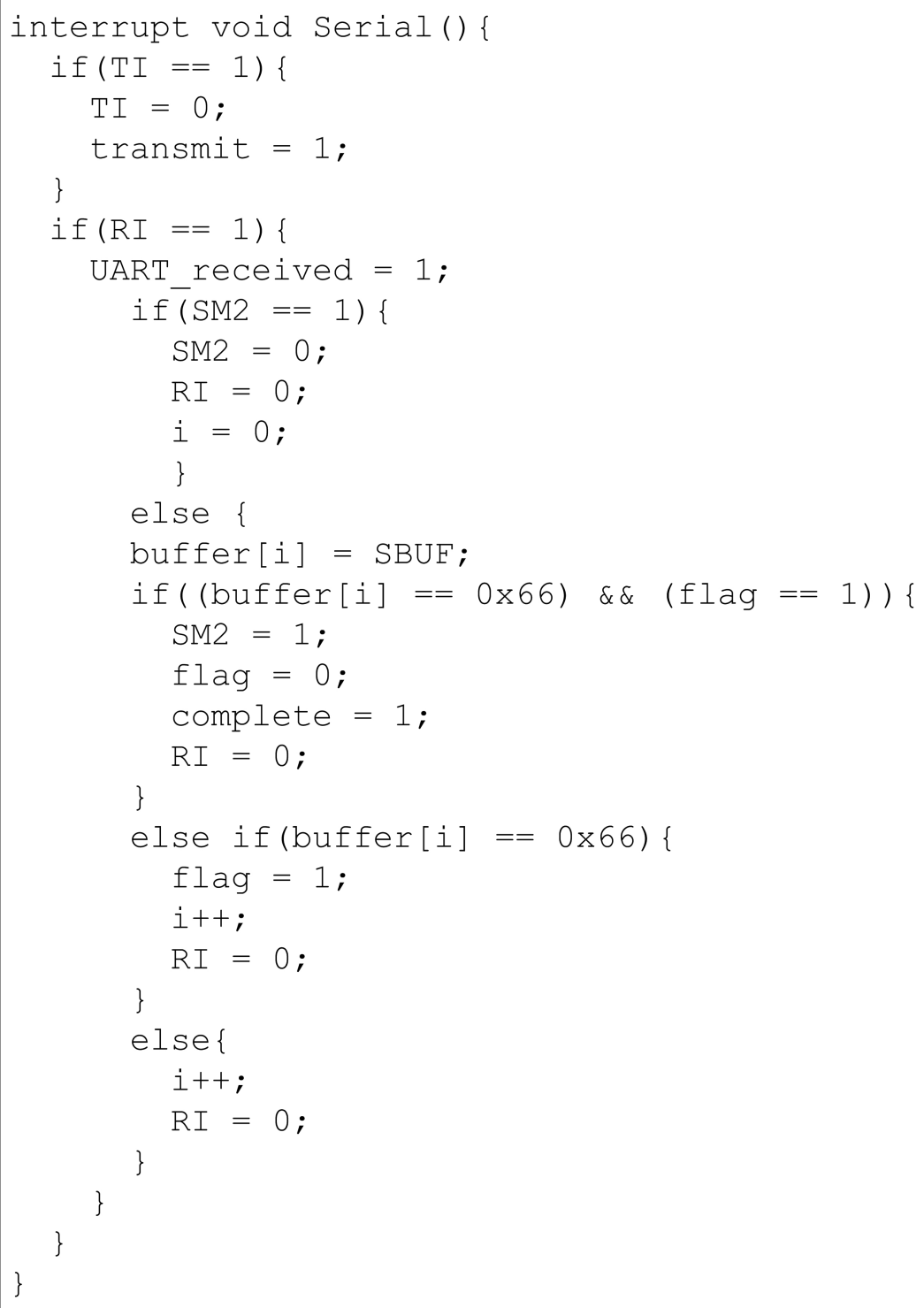




\section{B.6 Micro-controller PCA Configuration}

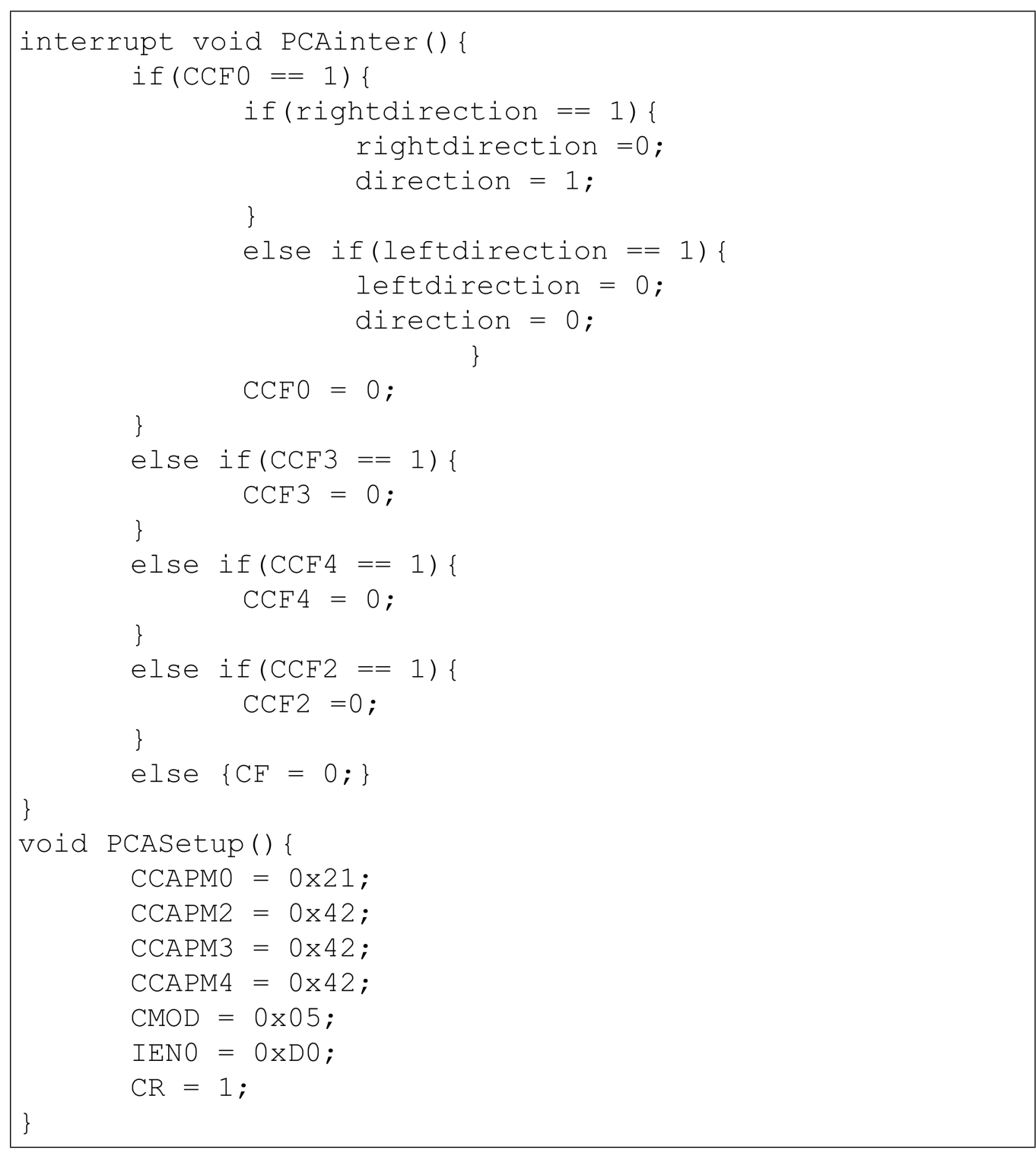




\section{B.7 Micro-controller Serial Functions}

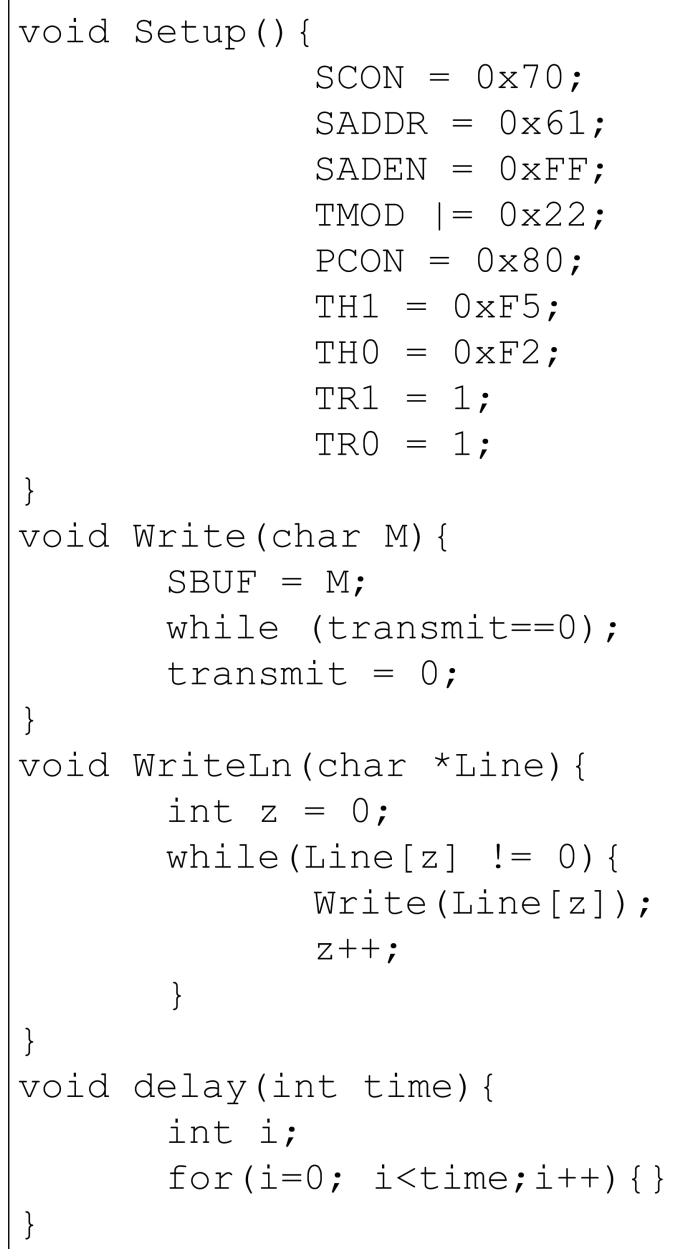




\section{B.8 Micro-controller Main Function 1}

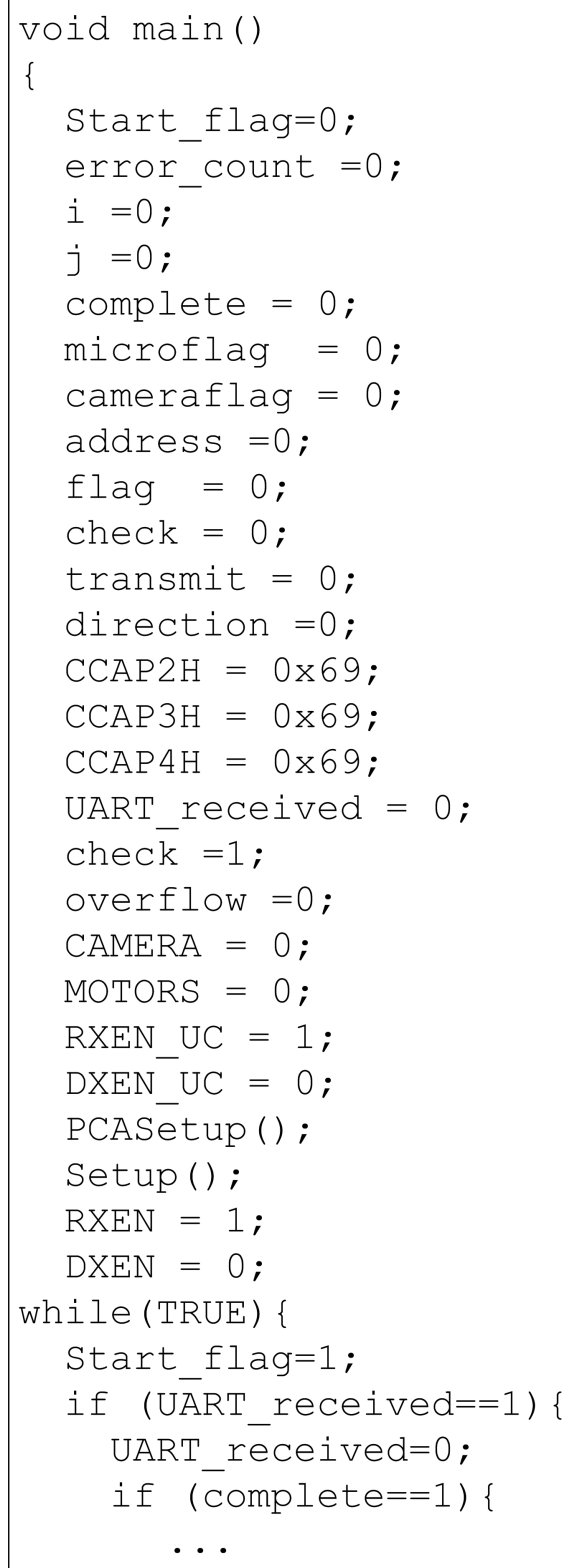




\section{B.9 Micro-controller Main Function 2}

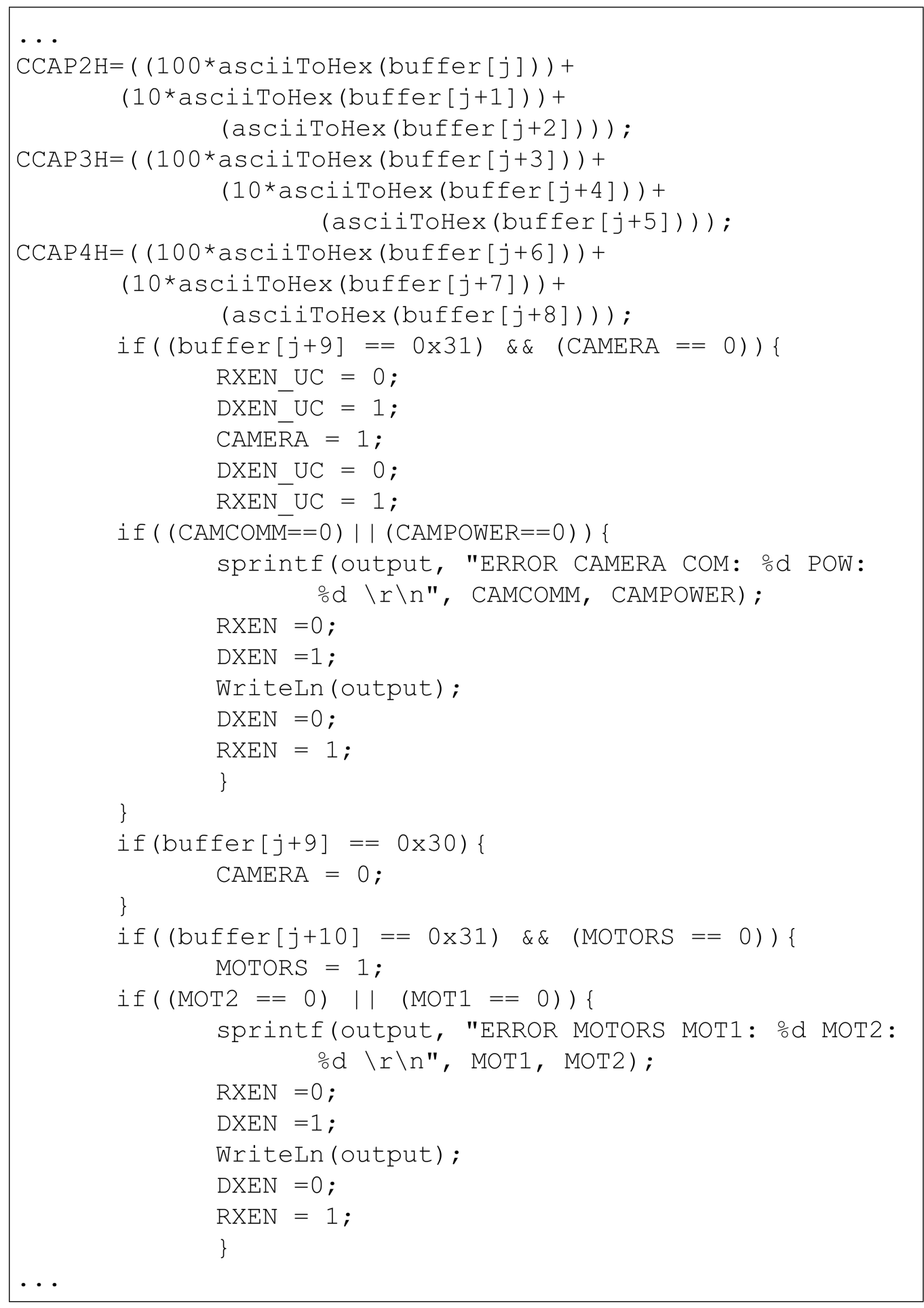




\section{B.10 Micro-controller Main Function 3}

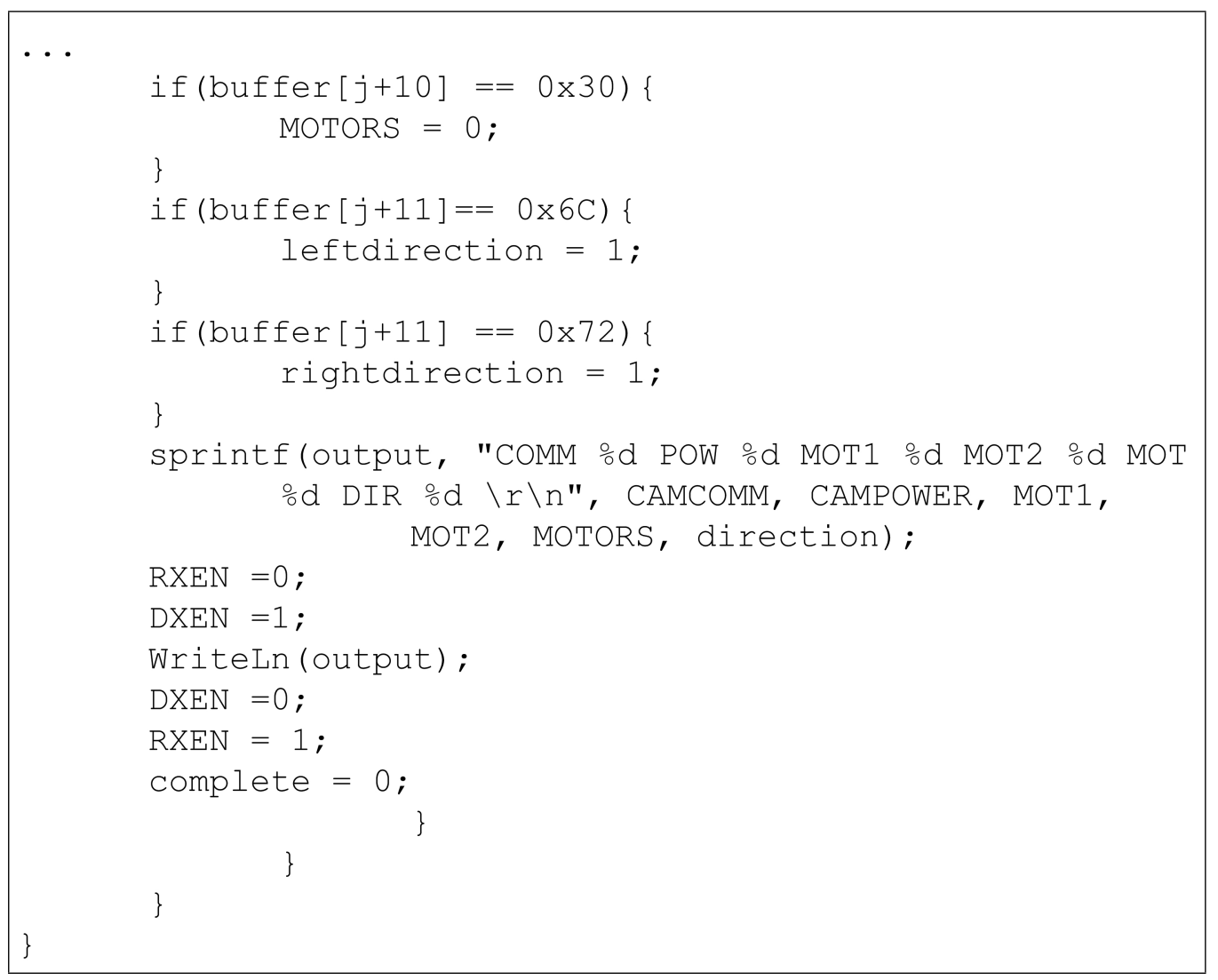

